\title{
ON THE EVOLUTION OF DUST MINERALOGY, FROM PROTOPLANETARY DISKS TO PLANETARY SYSTEMS
}

\author{
Isa Oliveira ${ }^{1}$, Johan Olofsson ${ }^{2}$, Klaus M. Pontoppidan ${ }^{3,4}$, Ewine F. van Dishoeck ${ }^{1,5}$, \\ Jean-Charles Augereau ${ }^{6}$, and Bruno Merín ${ }^{7}$ \\ ${ }^{1}$ Leiden Observatory, Leiden University, P.O. Box 9513, 2300 RA Leiden, The Netherlands; oliveira@ strw.leidenuniv.nl \\ 2 Max-Planck Institut für Astronomie, Königstuhl 17, 69117 Heidelberg, Germany \\ ${ }^{3}$ California Institute of Technology, Division for Geological and Planetary Sciences, MS 150-21, Pasadena, CA 91125, USA \\ ${ }^{4}$ Space Telescope Science Institute, Baltimore, MD 21218, USA \\ ${ }^{5}$ Max-Planck Institut für Extraterrestrische Physik, Giessenbachstrasse 1, 85748 Garching, Germany \\ ${ }^{6}$ UJF-Grenoble 1/CNRS-INSU, Institut de Planétologie et d'Astrophysique de Grenoble (IPAG) UMR 5274, Grenoble, F-38041, France \\ ${ }^{7}$ Herschel Science Center, European Space Agency (ESA), P.O. Box 78, 28691 Villanueva de la Cañada (Madrid), Spain \\ Received 2010 October 29; accepted 2011 April 5; published 2011 May 24
}

\begin{abstract}
Mineralogical studies of silicate features emitted by dust grains in protoplanetary disks and solar system bodies can shed light on the progress of planet formation. The significant fraction of crystalline material in comets, chondritic meteorites, and interplanetary dust particles indicates a modification of the almost completely amorphous interstellar medium dust from which they formed. The production of crystalline silicates, thus, must happen in protoplanetary disks, where dust evolves to build planets and planetesimals. Different scenarios have been proposed, but it is still unclear how and when this happens. This paper presents dust grain mineralogy (composition, crystallinity, and grain size distribution) of a complete sample of protoplanetary disks in the young Serpens cluster. These results are compared to those in the young Taurus region and to sources that have retained their protoplanetary disks in the older Upper Scorpius and $\eta$ Chamaeleontis stellar clusters, using the same analysis technique for all samples. This comparison allows an investigation of the grain mineralogy evolution with time for a total sample of 139 disks. The mean cluster age and disk fraction are used as indicators of the evolutionary stage of the different populations. Our results show that the disks in the different regions have similar distributions of mean grain sizes and crystallinity fractions $(\sim 10 \%-20 \%)$ despite the spread in mean ages. Furthermore, there is no evidence of preferential grain sizes for any given disk geometry nor for the mean cluster crystallinity fraction to increase with mean age in the 1-8 Myr range. The main implication is that a modest level of crystallinity is established in the disk surface early on $(\leqslant 1 \mathrm{Myr})$, reaching an equilibrium that is independent of what may be happening in the disk midplane. These results are discussed in the context of planet formation, in comparison with mineralogical results from small bodies in our own solar system.
\end{abstract}

Key words: circumstellar matter - infrared: stars - methods: statistical - protoplanetary disks - stars: pre-main sequence

Online-only material: color figures

\section{INTRODUCTION}

Protoplanetary disks originate from dense cloud material consisting of sub- $\mu \mathrm{m}$ sized, almost completely amorphous interstellar medium (ISM) dust grains (Beckwith et al. 2000; Li \& Draine 2001; Kemper et al. 2004; Henning 2010). The dust and gas in these disks form the basic matter from which planets may form. At the same time, mineralogical studies of primitive solar system bodies suggest that a considerable fraction of the silicate grains in these objects are of crystalline nature (Wooden et al. 2007; Pontoppidan \& Brearley 2010, and references therein). It is then naturally implied that the crystallinity fraction increases, through thermal and chemical modification of these solids during the general planet formation process, commonly referred to in the literature as "disk evolution."

As time passes, the small dust responsible for the infrared (IR) excess observed around young stars is subjected to different processes that affect and will eventually determine how this progression will end. Planets and planetary systems have been observed around hundreds of stars other than the Sun, showing that this result is rather common (Udry \& Santos 2007). IR observations have revealed a great number of debris disks, composed of large planetesimal rocks and smaller bodies, around a variety of stars spanning a large range in spectral types and ages (Rieke et al. 2005; Bryden et al. 2006; Su et al. 2006; Gautier et al. 2007; Carpenter et al. 2009). A few debris disks are known to harbor planets (e.g., $\beta$ Pictoris and Fomalhaut; Lagrange et al. 2010; Kalas et al. 2008), although it is still unclear whether this is often true (Kóspál et al. 2009). The majority of main-sequence stars show no signs of planets or debris within the current observational limitations, however, indicating that the disks around such stars at the time of their formation have dissipated completely, leaving no dust behind to tell the story. Which processes are important and determinant for the aftermath of disk evolution are still under debate, and this topic has been the subject of many theoretical and observational studies over the last decade, stimulated largely by recent IR and (sub-)millimeter facilities.

Specifically on the subject of the mineralogical composition, spectra from the ground and the Infrared Space Observatory provided the first clues of a potential link between crystalline material in protoplanetary disks and comets. A great similarity was noted between the spectra of the disk around the Herbig star HD 100546 and that of comet Hale-Bopp (Crovisier et al. 1997; Malfait et al. 1998). More recently, the InfraRed Spectrograph (IRS, 5-38 $\mu \mathrm{m}$; Houck et al. 2004) on board the 
Spitzer Space Telescope allowed an unprecedented combination of high sensitivity and the ability to observe large numbers of disks, down to the brown dwarf limit. The shape of the silicate features probed by the IRS spectra at 10 and $20 \mu \mathrm{m}$ is affected by the composition, size, and structure of its emitting dust. Amorphous silicates show broad, smooth mid-IR features, while the opacities of crystalline grains show sharp features due to their large-scale lattice arrangement, such that even small fractions of crystalline grains produce additional structure in the silicate features (Min et al. 2005; Bouwman et al. 2008; Juhász et al. 2009; Olofsson et al. 2010). Because most protoplanetary disks are optically thick at optical and IR wavelengths, the silicate features observed in the mid-IR are generally emitted by dust in the optically thin disk surface only. To probe the disk midplane, observations at longer wavelengths are necessary. Additionally, the emission at 10 and $20 \mu \mathrm{m}$ has been shown to arise from different grain populations, probing different radii (KesslerSilacci et al. 2006; Olofsson et al. 2009, 2010). While the $10 \mu \mathrm{m}$ feature probes a warmer dust population, at $\leqslant 1$ AU for $\mathrm{T}$ Tauri stars, the dust emitting at $20 \mu \mathrm{m}$ is colder, further out and deeper into the disk (Kessler-Silacci et al. 2007).

Two methods have been proposed to explain the formation of crystal grains: thermal annealing of amorphous grains or vaporization followed by gas-phase condensation. Both methods require high temperatures (above $\sim 1000 \mathrm{~K}$; Fabian et al. 2000; Gail 2004), which is inconsistent with outer disk temperatures. However, crystalline grains have been observed in outer, as well as in inner disks (van Boekel et al. 2004). Large-scale radial mixing has been invoked to explain the presence of crystals at low temperatures in the outer disk (Bockelée-Morvan et al. 2000; Gail 2004; Ciesla 2009). A third proposed formation mechanism for crystal formation is that shock waves could locally heat amorphous silicates and crystallize them (Desch \& Connolly 2002; Harker \& Desch 2002).

From protoplanetary disks to comets, several authors have attempted to infer the dust composition from IRS spectra and laboratory data on amorphous and crystalline silicate dust, using a variety of analysis techniques. Whether for individual objects (Forrest et al. 2004; Merín et al. 2007; Pinte et al. 2008; Bouy et al. 2008), for mixed disk samples (Bouwman et al. 2001, 2008; Apai et al. 2005; van Boekel et al. 2005; Olofsson et al. 2009, 2010; Juhász et al. 2010), or systematic studies of the disk population of a given star-forming region (Sicilia-Aguilar et al. 2009; Watson et al. 2009; Sargent et al. 2009), it has been shown that a significant mass fraction of the dust in those disks must be in crystalline form. However, the many studies dealing with the mineralogical composition of dust to date focus on a specific region or object, failing to investigate the hypothesis that the crystallinity fraction is a measure of the evolutionary stage of a region. That is, no study in the literature has yet investigated an increase of crystallinity fraction with cluster age.

Mineralogical studies of solar system bodies show a range of crystallinity fractions. Evidence from primitive chondrites shows that the abundance of crystalline silicate material varies from nearly nothing up to 20\%-30\% (e.g., Acfer 094 and ALH77307; Pontoppidan \& Brearley 2010, and references therein). Oort cloud comets, with long periods and large distances from the Sun, have inferred crystallinity fractions up to 60\%-80\% (e.g., Hale-Bopp; Wooden et al. 1999, 2007). Jupiterfamily, or short-period, comets have lower fractions, up to $\sim 35 \%$ (e.g., 9P/Tempel 1, Harker et al. 2007; 81P/Wild 2, Zolensky et al. 2006). This discrepancy in fractions points to the existence of a radial dependence in crystallinity fraction in the protoplan- etary disk around the young Sun (Harker et al. 2005). It is important to note that those values are model dependent, and the use of large amorphous grains $(10-100 \mu \mathrm{m})$ can lead to systematically lower crystalline fractions (Harker et al. 2002). This is evident for Hale-Bopp, where Min et al. (2005) find a much lower fraction $(\sim 7.5 \%)$ than other authors, using a distribution of amorphous grain sizes up to $100 \mu \mathrm{m}$. What is clear is that even within the discrepancies, the crystallinity fractions derived for solar system bodies are appreciably higher than those derived for the ISM dust ( $<2 \%$; Kemper et al. 2004). Recent Spitzer data indicate further similarities between crystalline silicate features seen in comets or asteroids with those seen in some debris disks around solar mass stars (Beichman et al. 2006; Lisse et al. 2007, 2008). One proposed explanation is that the observed spectral features in the disk result from the catastrophic break-up of a single large body (a "super comet") which creates the small dust particles needed for detection. At the even earlier protoplanetary disk stage, there is limited observational evidence for radial gradients in crystallinity from mid-infrared interferometry data, with higher crystallinity fractions found closer to the young stars (van Boekel et al. 2004; Schegerer et al. 2008). All of this suggests that the crystallization occurs early in the disk evolution and is then incorporated into larger solid bodies.

Besides dust composition, the evolution of grain sizes is an essential indicator of disk evolution. The initially sub- $\mu \mathrm{m}$ size ISM grains must grow astounding 14-15 orders of magnitude in diameter if they are to form planets. If grains were to grow orderly and steadily, theoretical calculations predict disks to have fully dissipated their small grains within $\sim 10^{5}$ years (Weidenschilling 1980; Dullemond \& Dominik 2005). The fact that many disks a few Myr old are observed to have small grains (Hernández et al. 2008) poses a serious problem for the paradigm that grain growth is a steady, monotonic process in disk evolution and planet formation. Additionally, small dust has been observed in the surface layers of disks in clusters of different ages and environments for hundreds of systems. The implication, as discussed most recently by Oliveira et al. (2010) and Olofsson et al. (2010), is that small grains must be replenished by fragmentation of bigger grains and that an equilibrium between grain growth and fragmentation is established. Oliveira et al. (2010) have shown that this equilibrium is maintained over a few million years, as long as the disks are optically thick, and is independent of the population or environment studied.

In this paper, we present a comprehensive study of the mineralogical composition of disks around stars in young star-forming regions (where most stars are still surrounded by optically thick disks) and older clusters (where the majority of disks have already dissipated). Correlating the results on mean size and composition of dust grains per region, obtained in a homogeneous way using the same methodology, with the properties of small bodies in our own solar system can put constraints on some of the processes responsible for disk evolution and planet formation. The Serpens Molecular Cloud, whose complete flux-limited young stellar object (YSO) population has been observed by the IRS instrument (Oliveira et al. 2010), is used as a prototype of a young star-forming region, together with Taurus, the best-studied region to date. The sources that have retained their protoplanetary disks in the $\eta$ Chamaeleontis and Upper Scorpius clusters are used to probe the mineralogy in the older bin of disk evolution.

Section 2 describes the YSO samples in the four regions mentioned. The Spitzer IRS observations and reduction are explained. The spectral decomposition method B2C (Olofsson 
Table 1

Characteristics of the Star-forming Regions Presented in This Work

\begin{tabular}{lcccc}
\hline \hline Region & Dist. (pc) & Mean Age (Myr) & Disk Fraction & Ref. \\
\hline Serpens & $259-415$ & $2-6$ & $\ldots$ & 1,2 \\
Taurus & 140 & $\sim 2$ & $\sim 60 \%$ & $3,4,5$ \\
Up Sco & 145 & $\sim 5$ & $\sim 17 \%$ & $6,7,8$ \\
$\eta$ Cha & 97 & $\sim 6$ & $\sim 40 \%$ & $9,10,11$
\end{tabular}

References. (1) The distance to Serpens is still under debate, different methods yield distances ranging from 259 (Straizys et al. 1996) to 415 pc (Dzib et al. 2010); (2) from Oliveira et al. 2009, using $d=259$ pc; (3) from Kenyon et al. 1994; (4) from Hartmann et al. 2001; (5) from Luhman et al. 2010; (6) from de Zeeuw et al. 1999; (7) from Blaauw 1978; (8) from Carpenter et al. 2006; (9) from Mamajek et al. 1999; (10) from Luhman \& Steeghs 2004; (11) from Megeath et al. 2005.

et al. 2010) is briefly introduced in Section 3, and its results for individual and mean cluster grain sizes and composition are shown in Section 4. In Section 5, the results are discussed in the context of time evolution. There we demonstrate that no evolution is seen in either mean grain sizes or crystallinity fractions as clusters evolve from $\sim 1$ to $8 \mathrm{Myr}$. The implications for disk formation and dissipation, and planet formation are discussed. In Section 6 we present our conclusions.

\section{SPITZER IRS DATA}

The four regions presented here were chosen due to the availability of complete sets of IRS spectra of their IR-excess sources, while spanning a wide range of stellar characteristics, environment, mean ages, and disk fractions (the disk fraction of Serpens is still unknown; see Table 1).

The IRS spectra of a complete flux-limited sample of YSOs in the Serpens Molecular Cloud have been presented by Oliveira et al. (2010), based on program ID 30223 (PI: Pontoppidan). As detailed there, the spectra were extracted from the basic calibration data (BCD) using the reduction pipeline from the Spitzer Legacy Program "From Molecular Cores to PlanetForming Disks" (c2d; Lahuis et al. 2006). A similarly large YSO sample in the Taurus star-forming region has been presented by Furlan et al. (2006). IRS spectra of all 18 members of the $\eta$ Chamaeleontis cluster were first shown by Sicilia-Aguilar et al. (2009), while the spectra of 26 out of the 35 IR-excess sources in the Upper Scorpius OB association were shown by Dahm \& Carpenter (2009; the remaining nine objects were not known at the time the observations were proposed). For the latter three regions, the post-BCD data were downloaded from the SSC pipeline (version S18.4) and then extracted with the Spitzer IRS Custom Extraction software (SPICE, version 2.3) using the batch generic template for point sources. As a test, the IRS spectra of the YSOs in Serpens were also reduced using SPICE to ensure that both pipelines produce nearly identical results. On visual inspection, no discrepancies were found between the results from the two pipelines, all objects showed the exact same features in both spectra. The similarity in outputs is such that the effects on the spectral decomposition results are within the cited error bars.

Since the spectral decomposition method applied here aims to reproduce the silicate emission from dust particles in circumstellar disks, the sample has been limited to spectra that show clear silicate features. The few sources with polycyclic aromatic hydrocarbon $(\mathrm{PAH})$ emission have been excluded from the sample. PAH sources amount to less than $8 \%$ in low-mass star-forming regions (Geers et al. 2006; Oliveira et al. 2010). Furthermore,

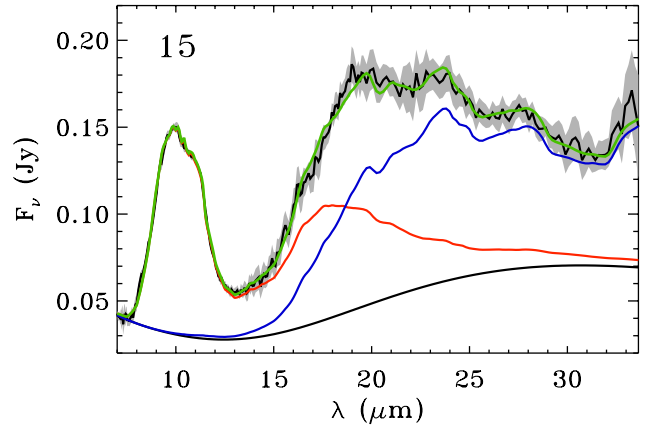

Figure 1. Example of the B2C modeling for object 15 in Serpens. The black line is the estimated continuum for this source. The red line is the fit to the warm component and the blue line is the fit to the cold component. The green line is the final fit to the entire spectrum. The original spectrum is shown in black with its uncertainties in light gray.

(A color version of this figure is available in the online journal.)

spectra with very low signal-to-noise ratios (S/Ns) are excluded from the analyzed sample in order to guarantee the quality of the results. In addition, for objects 114 and 137 in Serpens, and 04370+2559 and V955Tau in Taurus the warm component fit contributes to most of the spectrum, leaving very low fluxes to be fitted by the cold component. This produces large uncertainties in the cold component fit, and they are therefore not further used in the analysis. The low-S/N objects rejected amount to less than $10 \%$ of each of the Serpens and Taurus samples, so the statistical results derived here should not be affected by this removal. The final sample of 139 sources analyzed is composed of 60 objects in Serpens, 66 in Taurus, nine objects in Upper Scorpius, and four in $\eta$ Chamaeleontis. The statistical uncertainties of the spectra were estimated as explained in Olofsson et al. (2009).

The great majority of the objects studied here are low-mass stars (spectral types $\mathrm{K}$ and $\mathrm{M}$; see Table 5). The study of mineralogical evolution across stellar mass is not the focus of this paper. Such a study would require a separate paper, in which the same techniques are used for low- and intermediatemass stars. Thus, the statistical results derived in the following sections concern $\mathrm{T}$ Tauri stars, and not necessarily apply to intermediate-mass Herbig Ae/Be stars.

\section{SPECTRAL DECOMPOSITION AND THE B2C METHOD}

In order to reproduce the observed IRS spectra of these circumstellar disks, the B2C decomposition method, explained in detail and tested extensively in Olofsson et al. (2010), is applied. Two dust grain populations, or components, at different temperatures (warm and cold) are used in the method, in addition to a continuum emission. The warm component reproduces the $10 \mu \mathrm{m}$ feature, while the cold component reproduces the non-negligible residuals at longer wavelengths, over the full spectral range (see Figure 1). Each component, warm and cold, is the combination of five different dust species and three grain sizes for amorphous silicates or two grain sizes for crystalline silicates.

The three amorphous species are silicates of olivine stoichiometry $\left(\mathrm{MgFeSiO}_{4}\right)$, silicates of pyroxene stoichiometry $\left(\mathrm{MgFeSiO}_{6}\right)$, and silica $\left(\mathrm{SiO}_{2}\right)$. The two crystalline species are both $\mathrm{Mg}$-rich end members of the pyroxene and olivine groups, enstatite $\left(\mathrm{MgSiO}_{3}\right)$ and forsterite $\left(\mathrm{Mg}_{2} \mathrm{SiO}_{4}\right)$. As further explained in Olofsson et al. (2010), the theoretical opacities of the amorphous species are computed assuming homogeneous 
spheres (Mie theory), while those for the crystalline species use the distribution of hollow spheres (DHS; Min et al. 2005) theory so that irregularly shaped particles can be simulated.

In addition, the three grain sizes used are $0.1,1.5$, and $6.0 \mu \mathrm{m}$, representing well the spectroscopic behavior of very small, intermediate-sized and large grains. For the crystalline species, however, the code is limited to only two grain sizes $(0.1$ and $1.5 \mu \mathrm{m})$. This restriction is imposed because large crystalline grains are highly degenerate with large amorphous grains (as can be seen in Figure 1 of Olofsson et al. 2010), and because the production of large $6.0 \mu \mathrm{m}$ pure crystals is not expected via thermal annealing (Gail 2004).

The B2C method itself consists of three steps. First, the continuum is estimated and subtracted from the observed spectrum. The adopted continuum is built by using a power-law plus a blackbody at temperature $T_{\text {cont }}$. The power-law represents the mid-IR tail of emission from the star and inner disk rim. The blackbody is designed to contribute at longer wavelengths and is therefore constrained to be less than $150 \mathrm{~K}$. Each dust component is then fitted separately to the continuum-subtracted spectrum.

The second step is to fit the warm component to reproduce the $10 \mu \mathrm{m}$ silicate feature between $\sim 7.5$ and $13.5 \mu \mathrm{m}$. This is done by summing up the 13 mass absorption coefficients $\left(N_{\text {species }}=5, N_{\text {sizes }}=3\right.$ or 2 , for amorphous and crystalline species, respectively), multiplied by a blackbody $B_{v}\left(T_{\mathrm{w}}\right)$ at a given warm temperature $T_{\mathrm{w}}$.

The third step is to fit the residuals, mostly at longer wavelengths, over the entire spectral range $(5-35 \mu \mathrm{m})$. This is done in a similar manner, for a given cold temperature $T_{\mathrm{c}}$. The final fit is a sum of the three fits described, as can be seen in Figure 1. The entire fitting process is based on a Bayesian analysis, combined with a Monte Carlo Markov chain, in order to randomly explore the space of free parameters. The resulting mean mass-average grain size is the sum of all sizes fitted, each size being weighted by their corresponding masses, as

$$
\left\langle a_{\mathrm{warm} / \mathrm{cold}}\right\rangle=\left(\sum_{j=1}^{N_{\text {sizes }}} a_{j} \sum_{i=1}^{N_{\text {species }}} M_{\mathrm{w} / \mathrm{c}, i}^{j}\right) \times\left(\sum_{j=1}^{N_{\text {sizes }}} \sum_{i=1}^{N_{\text {species }}} M_{\mathrm{w} / \mathrm{c}, i}^{j}\right)^{-1},
$$

where $a_{1}=0.1 \mu \mathrm{m}$ (small grains), $a_{2}=1.5 \mu \mathrm{m}$ (intermediatesized grains), and $a_{3}=6 \mu \mathrm{m}$ (large grains). Further details and tests of the $\mathrm{B} 2 \mathrm{C}$ procedure can be found in Olofsson et al. (2010). That paper also demonstrates that the procedure is robust for statistical samples, and that the relative comparisons between samples, which are the focus of this paper, should not suffer from the assumptions that enter in the procedure. The robustness of the procedure is evaluated by fitting synthetic spectra and is discussed in detail in their Appendix A. The influence of the continuum estimate is also discussed, especially for the cold component for both grain sizes and crystallinity fractions, and it is shown that prescriptions that do not use large $6 \mu \mathrm{m}$ grains (which are, to some degree, degenerate with the continuum) give fits that are not so good.

For the amorphous grains, the B2C procedure uses the Mie scattering theory to compute mass absorption coefficients. However, Min et al. (2007) found that they could best reproduce the extinction profile toward the galactic center using the DHS scattering theory, with a maximum filling factor of 0.7 . The most striking difference between Mie and DHS mass absorption coefficients is seen for the $\mathrm{O}-\mathrm{Si}-\mathrm{O}$ bending mode around
Table 2

B2C Mean Composition of Each Star-forming Region

\begin{tabular}{lcccc}
\hline \hline Region & \multicolumn{1}{c}{ Oli/Pyr ${ }^{\mathrm{a}}$} & Silica & Forsterite & Enstatite \\
\hline \multicolumn{5}{c}{ Warm component } \\
\hline Serpens & $81.3 \% \pm 11.7 \%$ & $7.8 \% \pm 6.5 \%$ & $5.8 \% \pm 4.9 \%$ & $5.2 \% \pm 4.9 \%$ \\
Taurus & $79.4 \% \pm 9.4 \%$ & $9.6 \% \pm 7.0 \%$ & $4.4 \% \pm 3.3 \%$ & $6.5 \% \pm 4.5 \%$ \\
Up Sco & $89.7 \% \pm 4.7 \%$ & $3.5 \% \pm 3.4 \%$ & $2.6 \% \pm 1.1 \%$ & $4.1 \% \pm 3.1 \%$ \\
$\eta$ Cha & $75.0 \% \pm 13.4 \%$ & $7.8 \% \pm 4.6 \%$ & $6.8 \% \pm 4.0 \%$ & $10.3 \% \pm 9.8 \%$ \\
\hline \multicolumn{5}{c}{ Cold component } \\
\hline Serpens & $68.0 \% \pm 20.1 \%$ & $14.4 \% \pm 12.3 \%$ & $9.5 \% \pm 9.5 \%$ & $8.0 \% \pm 8.0 \%$ \\
Taurus & $64.7 \% \pm 14.3 \%$ & $21.3 \% \pm 11.4 \%$ & $8.6 \% \pm 8.6 \%$ & $5.3 \% \pm 5.3 \%$ \\
\hline
\end{tabular}

Note. ${ }^{a}$ Amorphous olivine and pyroxene combined.

$20 \mu \mathrm{m}$. Here, we investigate the influence of the use of DHS instead of Mie for amorphous grain with an olivine or pyroxene stoichiometry. We conducted tests on a sub-sample of 30 objects (15 in Serpens and 15 in Taurus). The conclusion of such tests is that it has a small influence on the quantities we discuss in this study. For the warm component of the 30 objects, we find a change in the mean crystallinity fraction of $-1.6 \%$ (the mean crystallinity for this sub-sample using DHS is $9.3 \%$ versus $10.9 \%$ using Mie), which is in the range of uncertainties claimed in this study. We also computed the mean slope of grain size distributions to gauge the effect of using DHS on grain sizes. On average, the grain size distribution indices are steeper by $\sim 0.2$ (with a mean slope of -3.01 for this sub-sample using DHS versus -2.80 using Mie). Therefore, our main conclusions are preserved for the warm component. Concerning the cold component, the inferred crystallinity fraction using DHS is $22.5 \%$ versus $15.1 \%$ with Mie, a mean increase of $7.4 \%$. For the mean slope of grain size distributions, a negligible decrease is found ( -3.07 using DHS versus -3.01 for Mie). Again, the differences found are within our significant errors for the cold component and do not change any of our conclusions.

It is important to note that the $\mathrm{S} / \mathrm{N}$ generally degrades at longer wavelengths when compared to shorter wavelengths. The lower S/Ns reflect on the cold component fits and will most likely result in larger uncertainties. We evaluate that the fits to the cold component are reliable and add important information on the dust mineralogy (albeit with larger uncertainties) and thus those results are included in the following discussion.

\section{RESULTS}

The IRS spectra of the 139 YSOs with IR excess discussed in Section 2 were fitted with the B2C spectral decomposition procedure. The relative abundances derived for all objects are shown in the Appendix. The $\mathrm{S} / \mathrm{N}$ drops considerably for the long wavelength module of some of the objects studied (including all objects in Upper Scorpius and $\eta$ Chamaeleontis). For this reason, the cold component could not be satisfactorily fitted and no results for this component are presented for these sources (see the Appendix).

Due to the large number of objects, these results allow statistical studies on both the mineralogy and size distribution of the grains that compose the optically thin surface layers of disks in each cluster studied. The mean abundances of each species per region are presented in Table 2, where it can be seen that the majority of the dust studied is of amorphous form. In Table 3, the mean mass-average grain sizes and crystallinity fractions per region are shown. Mean sizes are in the range 1-3 $\mu \mathrm{m}$, without significant difference between regions. These results are discussed in detail in the following sections. 
Table 3

B2C Mean Grain Size and Crystallinity Parameters for Each Star-forming Region

\begin{tabular}{|c|c|c|c|c|c|c|c|}
\hline Region & Number & $\begin{array}{c}\left\langle a_{\mathrm{warm}}\right\rangle \\
(\mu \mathrm{m})\end{array}$ & $\begin{array}{c}\left\langle a_{\text {cold }}\right\rangle \\
(\mu \mathrm{m})\end{array}$ & $\left\langle\alpha_{\text {warm }}\right\rangle$ & $\left\langle\alpha_{\text {cold }}\right\rangle$ & $\begin{array}{c}\left\langle C_{\text {warm }}\right\rangle \\
(\%)\end{array}$ & $\begin{array}{c}\left\langle C_{\text {cold }}\right\rangle \\
(\%)\end{array}$ \\
\hline Serpens & 60 & $2.9 \pm 1.3$ & $1.9 \pm 0.6$ & $-2.75 \pm 0.39$ & $-3.16 \pm 0.18$ & $11.0 \pm 6.9$ & $17.5 \pm 12.4$ \\
\hline Taurus & 66 & $2.6 \pm 0.9$ & $2.4 \pm 0.6$ & $-2.83 \pm 0.31$ & $-3.02 \pm 0.15$ & $10.9 \pm 5.6$ & $13.9 \pm 10.1$ \\
\hline Up Sco & 9 & $3.1 \pm 1.5$ & $\ldots$ & $-3.33 \pm 0.18$ & $\ldots$ & $6.8 \pm 3.3$ & $\ldots$ \\
\hline$\eta$ Cha & 4 & $1.3 \pm 0.4$ & $\ldots$ & $-2.71 \pm 0.39$ & $\ldots$ & $17.1 \pm 10.6$ & $\ldots$ \\
\hline
\end{tabular}

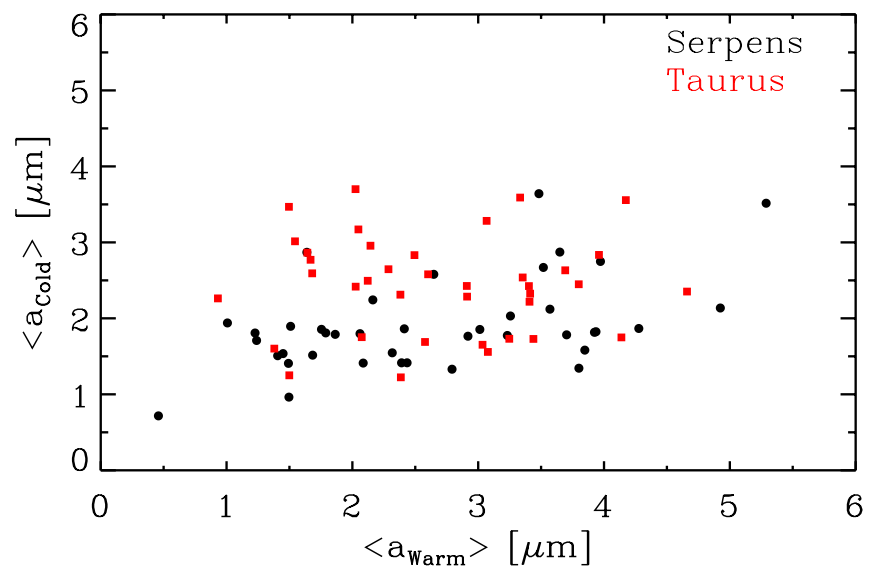

Figure 2. Mass-averaged mean grain sizes for the warm $\left(\left\langle a_{\text {warm }}\right\rangle\right)$ and cold $\left(\left\langle a_{\text {cold }}\right\rangle\right)$ components. Black dots are the objects in Serpens, and red squares are the objects in Taurus.

(A color version of this figure is available in the online journal.)

It is important to note that the comparison of results derived here for the different regions is valid because the same method, with exact same species, is used for all sources. The comparison of samples analyzed in distinct ways can lead to differences in results that do not correspond to real differences in composition. Nevertheless, in Section 4.5 the results presented here are compared to literature results for the same objects, when available, with generally good agreement.

\subsection{Grain Sizes}

The mean mass-averaged grain sizes for the warm $\left(\left\langle a_{\mathrm{warm}}\right\rangle\right)$ and cold $\left(\left\langle a_{\text {cold }}\right\rangle\right)$ components are shown in Figure 2, for Serpens and Taurus (for the objects in Upper Sco and $\eta$ Cha no results for the cold component are available; see the Appendix). It is seen that the two clouds overlap greatly, and that the grain sizes derived from the different temperature components do not seem to correlate. To quantify this correlation, a Kendall $\tau$ correlation coefficient can be computed together with its associated probability $P$ (between 0 and 1). $\tau=1(-1)$ defines a perfect correlation (anti-correlation), and $\tau=0$ means that the data sets are completely independent. A small $P$, on the other hand, testifies to how tight the correlation is. For the warm and cold mean mass-averaged grain sizes for both clouds, $\tau$ is found to be 0.14 , with $P=0.07$. This lack of correlation indicates that different processes are likely responsible for regulating the size distribution at different radii (Olofsson et al. 2010).

Although the average grain size in the warm component is bigger than that in the cold component within a given starforming region, as shown in Table 3, this difference is mostly not significant. However, Figures 2 and 3 clearly show a difference between the range of grain sizes spanned in both components, with $\left\langle a_{\text {cold }}\right\rangle$ never reaching near the largest grain size modeled $(6.0 \mu \mathrm{m})$ for any object. A possible explanation for larger grains at smaller radii, suggested by Sargent et al. (2009), is that grains coagulate faster in the inner disk where dynamical timescales are shorter. However, as discussed by Oliveira et al. (2010) and in Section 5.1, the mean dust size at the disk surface is not regulated by grain growth alone, but also by fragmentation and vertical mix. This means that faster coagulation at smaller radii cannot be uniquely responsible for bigger grains in the inner disk. Future modeling should try to understand this difference in mean grain sizes observed.

Furthermore, Serpens and Taurus occupy an indistinguishable locus in Figure 2, explicitly seen in Figure 3. A two sample Kolmogorov-Smirnov test (K-S test) was performed and the results show that the null hypothesis that the two distributions come from the same parent population cannot be rejected to any significance (14\%). The older regions, although lacking statistical significance, show a distribution of mass-average grain sizes in the same range probed by the young starforming regions (Figure 3). This supports the evidence that the size distribution of the dust in the surface layers of disks is statistically the same independent of the population studied (Oliveira et al. 2010).

The results here confirm those from Olofsson et al. (2010) that the mean differential grain size distributions slope for the three grain sizes considered are shallower than the reference MRN differential size distribution $(\alpha=-3.5)$. The mean grain size distribution slopes $(\alpha)$ for each region can be found in Table 3 .

\subsection{Disk Geometry}

The amount of IR excess in a disk is directly related to its geometry (Kenyon \& Hartmann 1987; Meeus et al. 2001; Dullemond et al. 2001). Specifically using the IRS spectra, disk geometry can be inferred from the flux ratio between 30 and $13 \mu \mathrm{m}\left(F_{30} / F_{13}\right.$; Brown et al. 2007; Oliveira et al. 2010; Merín et al. 2010). A flared geometry $\left(1.5 \lesssim F_{30} / F_{13} \lesssim 5\right)$, with considerable IR excess and small dust, allows the uppermost dust layers to intercept stellar light at both the inner and outer disks. For flat disks $\left(F_{30} / F_{13} \lesssim 1.5\right)$ with little IR excess, only the inner disk can easily intercept the stellar radiation as the outer disk is shadowed. Moreover, cold or transitional disks are interesting objects that present inner dust gaps or holes, producing a region with little or no near-IR excess (5 $\left.\lesssim F_{30} / F_{13} \lesssim 15\right)$. It is interesting to explore the effect of disk geometry on both the mean mass-average grain sizes and crystallinity fractions of the disks studied.

Figure 4 shows $F_{30} / F_{13}$ as a proxy for disk geometry compared with the mean mass-averaged grain sizes and crystallinity fractions for both components and all regions studied here. No preferential grain size (correlation coefficient $\tau=-0.14, P=$ 0.02 , and $\tau=0.07, P=0.33$ for warm and cold components, respectively) nor crystallinity fraction ( $\tau=0.09, P=0.10$ for the warm, and $\tau=-0.19, P=0.01$ for the cold component) is apparent for any given disk geometry. Similar scatter plots result for the mean mass-average grains sizes for only amorphous 

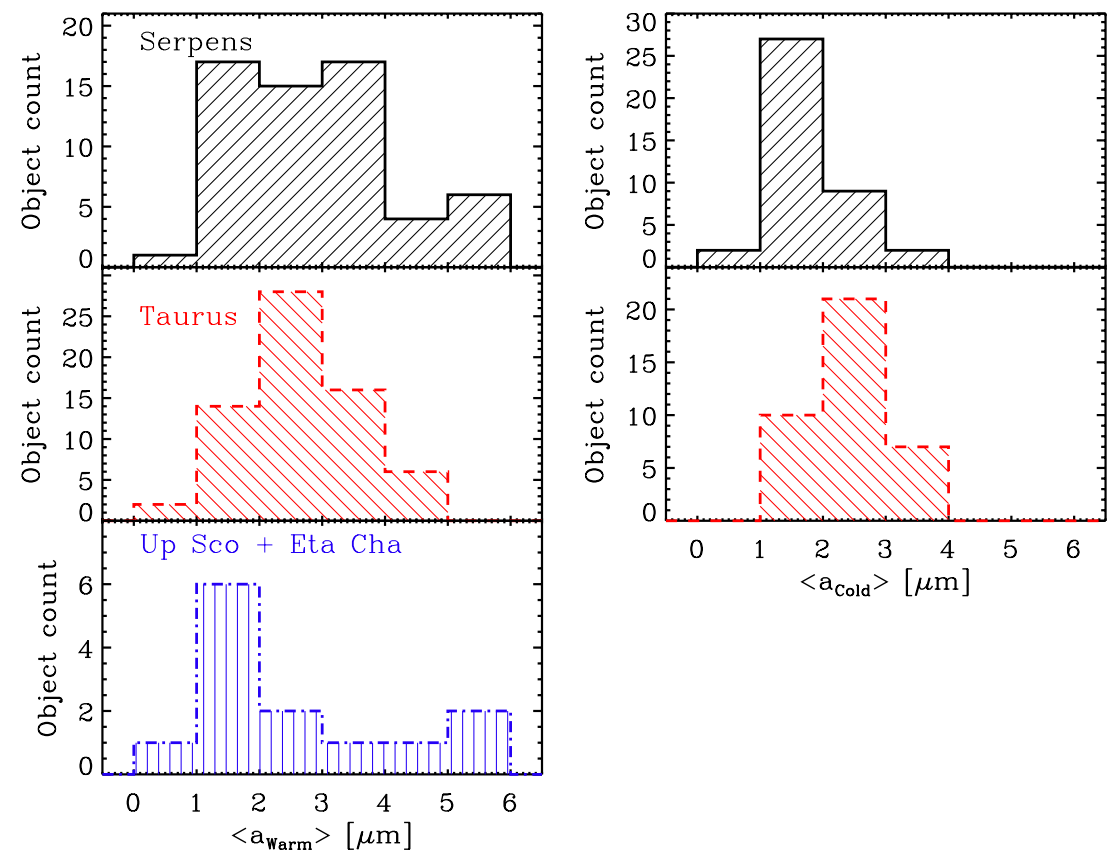

Figure 3. Distribution of mass-averaged mean grain sizes for the warm $\left(\left\langle a_{\text {warm }}\right\rangle\right.$, left panel $)$ and cold $\left(\left\langle a_{\text {cold }}\right\rangle\right.$, right panel $)$ components. Due to the low number statistics, the objects in Upper Sco and $\eta$ Cha have been merged together as an older cluster.

(A color version of this figure is available in the online journal.)
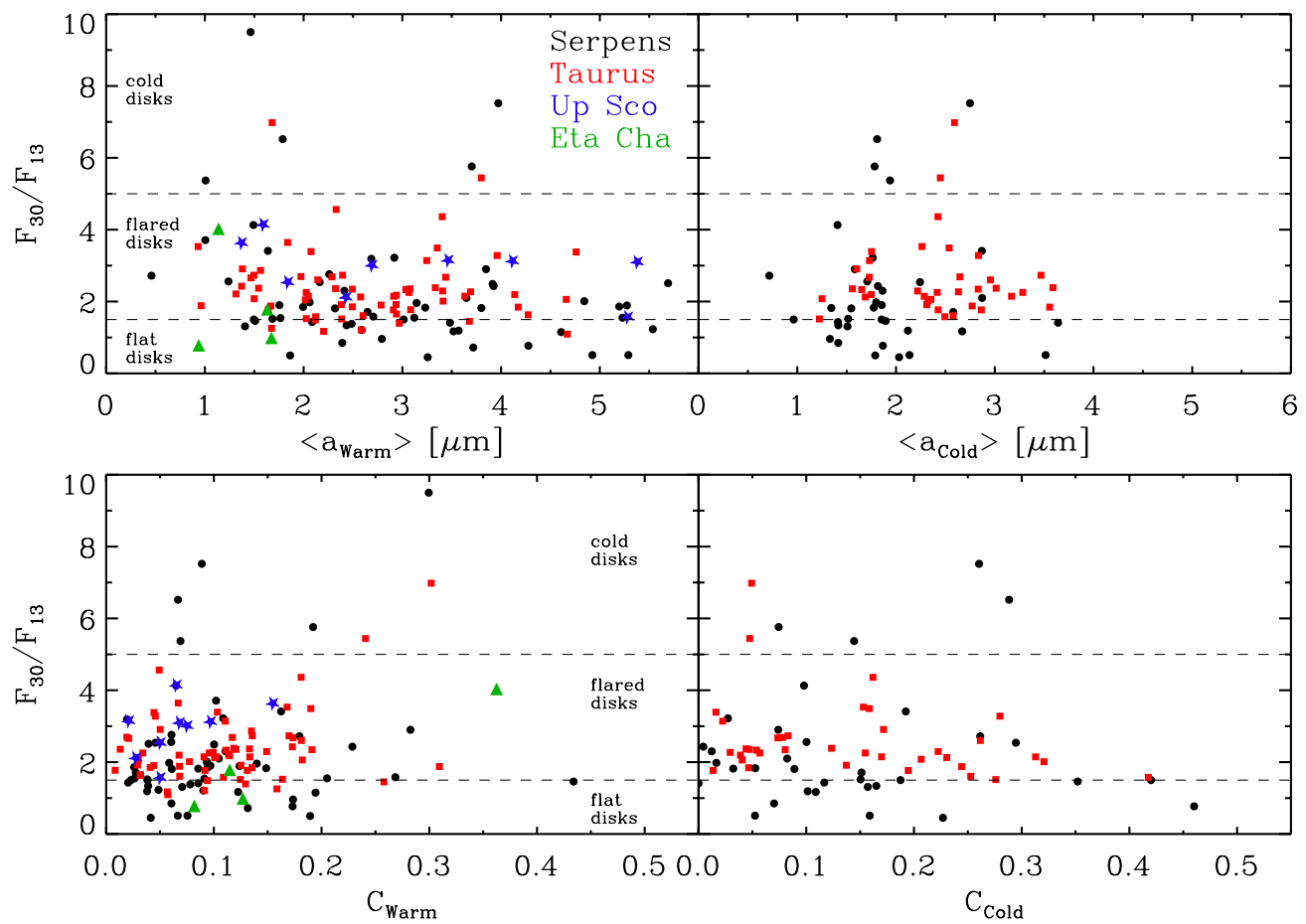

Figure 4. Top: flaring index $F_{30} / F_{13}$, used as a proxy for disk geometry, vs. warm (left) and cold (right) mass-averaged mean grain sizes. Bottom: $F_{30} / F_{13}$ vs. warm (left) and cold (right) crystallinity fractions. The YSOs in Serpens (black dots), Taurus (red squares), Upper Sco (blue stars), and $\eta$ Cha (green triangles) are compared. (A color version of this figure is available in the online journal.)

( $\tau=-0.12, P=0.08$ for the warm, and $\tau=0.13, P=0.11$ for the cold component) or only crystalline grains $(\tau=0.08$, $P=0.17$ for the warm, and $\tau=-0.13, P=0.10$ for the cold component). Furthermore, no clear separation is seen between the different regions studied. The statistically relevant samples in Serpens and Taurus define a locus where the majority of the objects are located in each plot, which is followed by the lower number statistics for older regions. Figure 4 therefore shows not only that grain size and crystallinity fraction are not a function of disk geometry, but also that younger and older regions show similar distributions of those two parameters.

\subsection{Crystallinity Fraction}

The crystallinity fractions derived from the warm and cold components ( $C_{\text {Warm }}$ and $C_{\text {Cold }}$, respectively) for Serpens and Taurus are show in Figure 5. No strong trend of warm and cold 


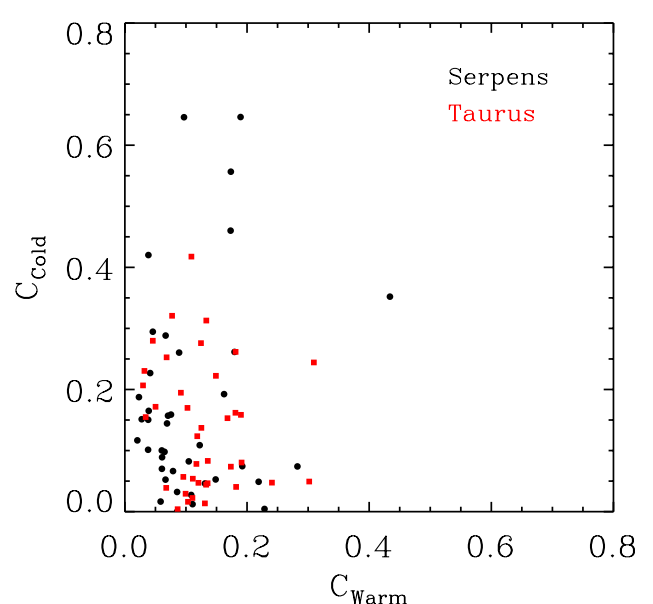

Figure 5. Crystalline fraction of the warm and cold components in Serpens (black dots) and Taurus (red squares).

(A color version of this figure is available in the online journal.)

crystallinity fractions increasing together is seen $(\tau=0.10$, with $P=0.10$ for the entire sample). This fact implies that, if an unique process is responsible for the crystallization of dust at all radii, this process is not occurring at the same rate in the innermost regions as further out in the disk. This is the opposite of the conclusion of Watson et al. (2009), who derive a correlation between inner and outer disk crystallinity from the simultaneous presence of the 11.3 and $33 \mu \mathrm{m}$ features. The opacities of the crystalline species are more complex than those two features alone, making the analysis here more complete than that of Watson et al. (2009). Our finding that the fraction of crystalline material in disk surfaces varies with radius can constrain some of the mechanisms for formation and distribution of crystals.

A wider spread in crystallinity fraction is observed for the cold component than for the warm component (Figure 6), which is reflected in the mean crystallinity fractions for each sample
(Table 3). This discrepancy could be real, or an artifact due to the $\mathrm{S} / \mathrm{N}$ being frequently lower at longer wavelengths (cold component) than that at shorter wavelengths (warm component), introducing a larger scatter. The difference in Serpens is more significant $\left(\left\langle C_{\text {warm }}\right\rangle \simeq 11.0 \%\right.$ and $\left.\left\langle C_{\text {cold }}\right\rangle \simeq 17.5 \%\right)$. The left panel of Figure 7 shows the cumulative fractions as functions of crystallinity fractions. Despite small differences between the warm (red line) and cold (blue line) components, the two cumulative fractions have similar behavior. If this difference is true, there is a small fraction of T Tauri disks with a higher cold (outer) than warm (inner) crystallinity fraction. This finding contrasts with that derived by van Boekel et al. (2004) for the disks around three Herbig stars. Their spatially resolved observations infer higher crystallinity fractions in the inner disks than in the outer disks, albeit based on only $10 \mu \mathrm{m}$ data. A larger sample of objects with good $\mathrm{S} / \mathrm{N}$ including both 10 and $20 \mu \mathrm{m}$ data is needed to better constrain this point. In addition, Figure 6 shows that younger and older clusters have similar distributions of crystallinity fractions.

As discussed by many authors, both the grain size and the degree of crystallinity affect the silicate features, therefore it is interesting to search for trends between these two parameters. In Figure 8, the mass-average grain sizes are compared to the crystallinity fraction for both warm (left panel) and cold (right panel) components. No obvious trends are seen in either component, neither any separation between regions. This result supports the discussion of Olofsson et al. (2010) that whatever processes govern the mean grain size and the crystallinity in disks, they are independent from each other.

\subsubsection{Enstatite versus Forsterite}

The disk models of Gail (2004) consider chemical equilibrium of a mixture of solid and gas at high temperatures, allowing radial mixing of material. These models predict a predominance of forsterite in the innermost regions of the disk, while enstatite dominates at lower temperatures (being converted from
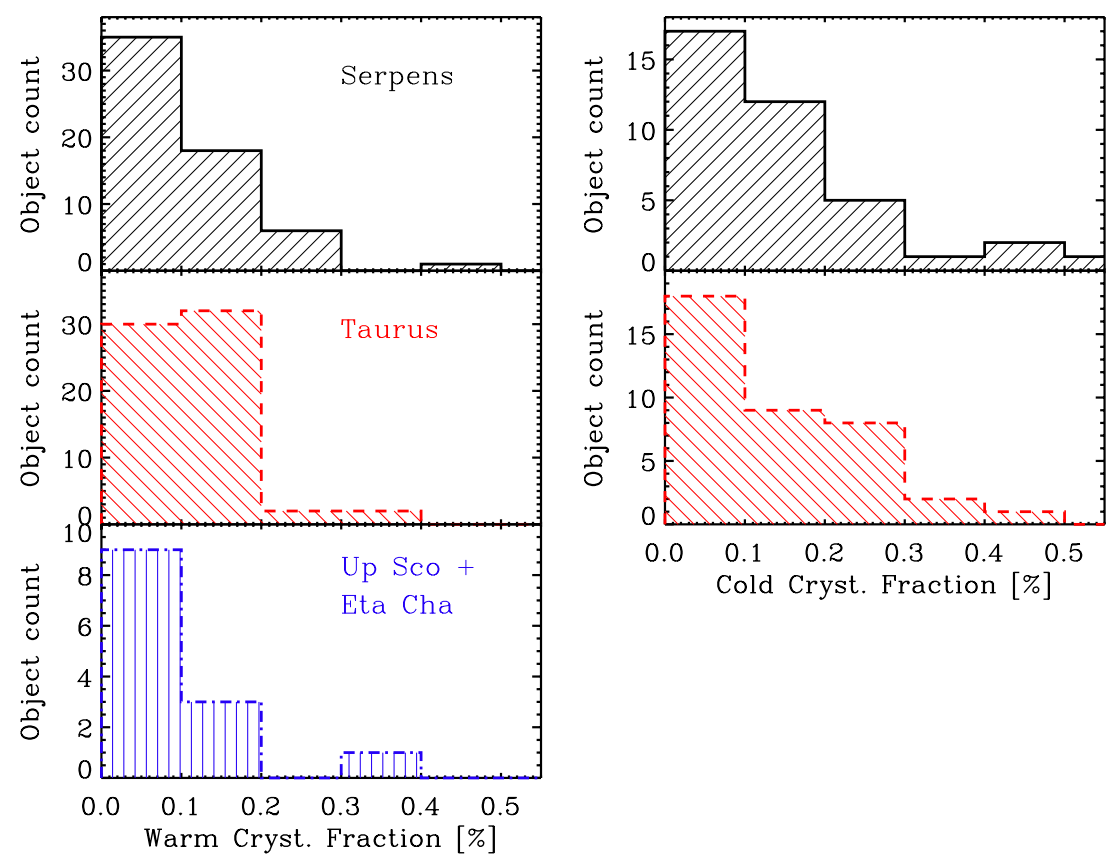

range of fractions are seen for all clusters.

(A color version of this figure is available in the online journal.) 


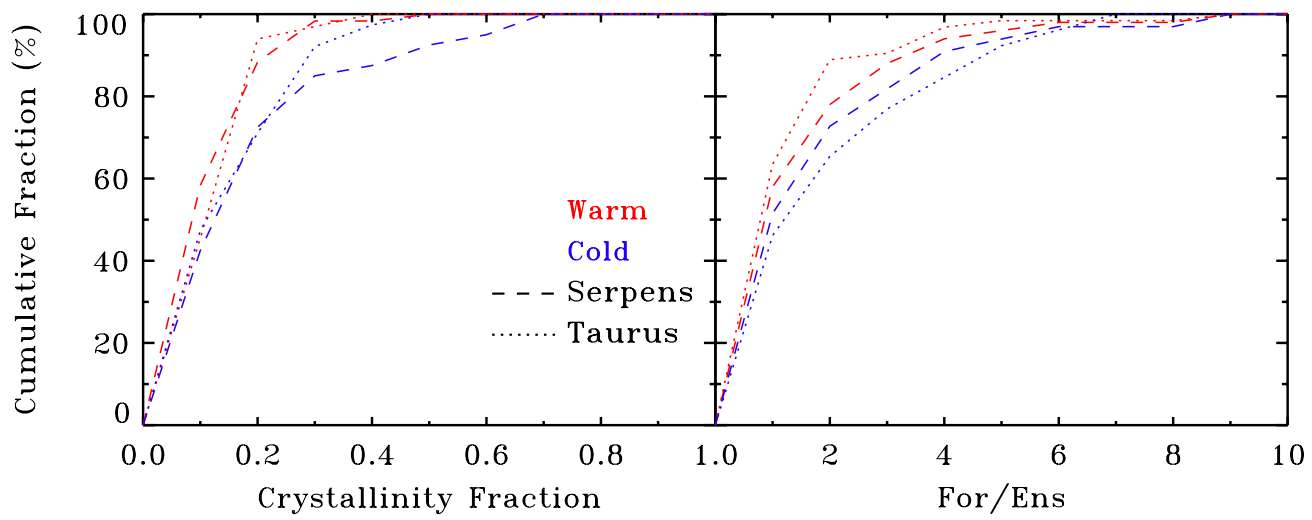

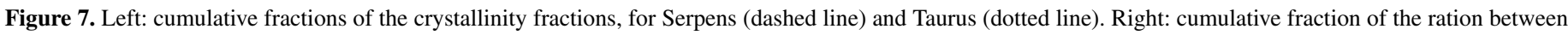
the forsterite and enstatite fractions, for Serpens (dashed line) and Taurus (dotted line). The warm component is shown in red while the cold component is blue. (A color version of this figure is available in the online journal.)
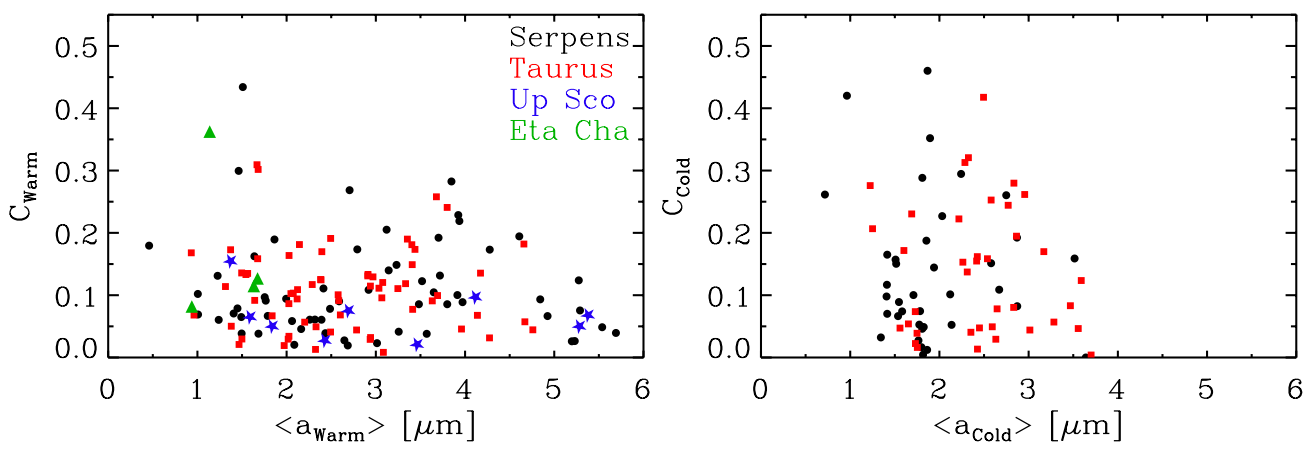

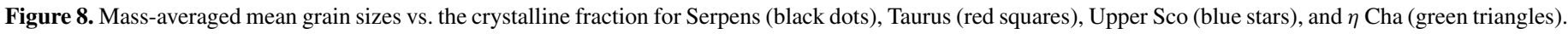
(A color version of this figure is available in the online journal.)

forsterite). From the observational point of view, data on disks around T Tauri (Bouwman et al. 2008) and Herbig Ae/Be stars (Juhász et al. 2010) have shown the opposite trend: enstatite is more concentrated in the inner disk, while forsterite dominates the colder, outer disk region. Bouwman et al. (2008) interpret this result as a radial dependence of the species formation mechanisms, or a non-equilibrium of the conditions under which the species formed, contrary to the model assumptions.

For the regions presented in this study, it can be seen in Table 2 for mean cluster values and in Table 5 for individual objects that the results derived from this study generally follow those of Bouwman et al. (2008), with more enstatite in the warm component and, to a lesser extent, more forsterite in the cold component. The right panel of Figure 7 illustrates this for the cumulative fraction of the forsterite over enstatite ratios for individual disks. However, this trend is not very significant given the uncertainties.

\subsection{The Silicate Strength-Shape Relation}

A correlation between the shape and the strength of the $10 \mu \mathrm{m}$ silicate feature from disks has been discussed extensively in the literature (van Boekel et al. 2003; Kessler-Silacci et al. 2006; Olofsson et al. 2009, 2010; Pascucci et al. 2009; Oliveira et al. 2010). Synthetic $10 \mu \mathrm{m}$ features generated for different grain sizes and compositions have been shown to fit well with observations, yielding grain size as the important parameter responsible for such a relationship. The degree of crystallinity of the dust also plays a role on the shape of this feature. However, as clearly shown for EX Lup (Ábrahám et al. 2009), and supported by models (Min et al. 2008; Olofsson et al. 2009), an increase in crystallinity fraction does not change the strength of the feature, even though its shape does change. Crystallinity is then understood as being responsible for the scatter in the strength-shape relationship, and not the relationship itself. As a result, the strength and shape of the $10 \mu \mathrm{m}$ silicate feature yield the typical size of the grains in the upper layers of the disk at a few AU from the star (Kessler-Silacci et al. 2007). The top panel of Figure 9 shows the results for Serpens, Taurus, Upper Sco, and $\eta$ Cha. The bottom panel presents the median values per region, indicating the 15-85 percentile ranges of the distributions. Overlaid are the models of Olofsson et al. (2009) for different grain sizes $(0.1-6.0 \mu \mathrm{m})$ generated for amorphous silicates of olivine and pyroxene stoichiometry, and a 50:50 mixture. The difference in mean ages does not correspond to a significant difference in mean grain sizes between the different regions.

With the mean grain sizes derived from the spectral decomposition, it is possible to further explore the validity of using the strength of the $10 \mu \mathrm{m}$ silicate feature to trace the sizes of grains in the surfaces of disks. The left panel of Figure 10 shows the correlation between $\left\langle a_{\mathrm{warm}}\right\rangle$ and $S_{\text {peak }}^{10 \mu \mathrm{m}}$ for all four samples. The Kendall $\tau$ coefficient of $-0.29, P=0.01$ supports the effectiveness of $S_{\text {peak }}^{10 \mu \mathrm{m}}$ as a proxy for grain sizes, with smaller values of $S_{\text {peak }}^{10 \mu \mathrm{m}}$ implying larger grain sizes.

On the other hand, it is also possible to test how the degree of crystallinity can influence the strength of the $10 \mu \mathrm{m}$ silicate feature. The lack of correlation between $C_{\text {warm }}$ and $S_{\text {peak }}^{10 \mu \mathrm{m}}(\tau=$ $-0.07, P=0.14$ ), shown in the right panel of Figure 10 for all samples, supports that the degree of crystallinity is not the dominant parameter setting the strength of the $10 \mu \mathrm{m}$ silicate 


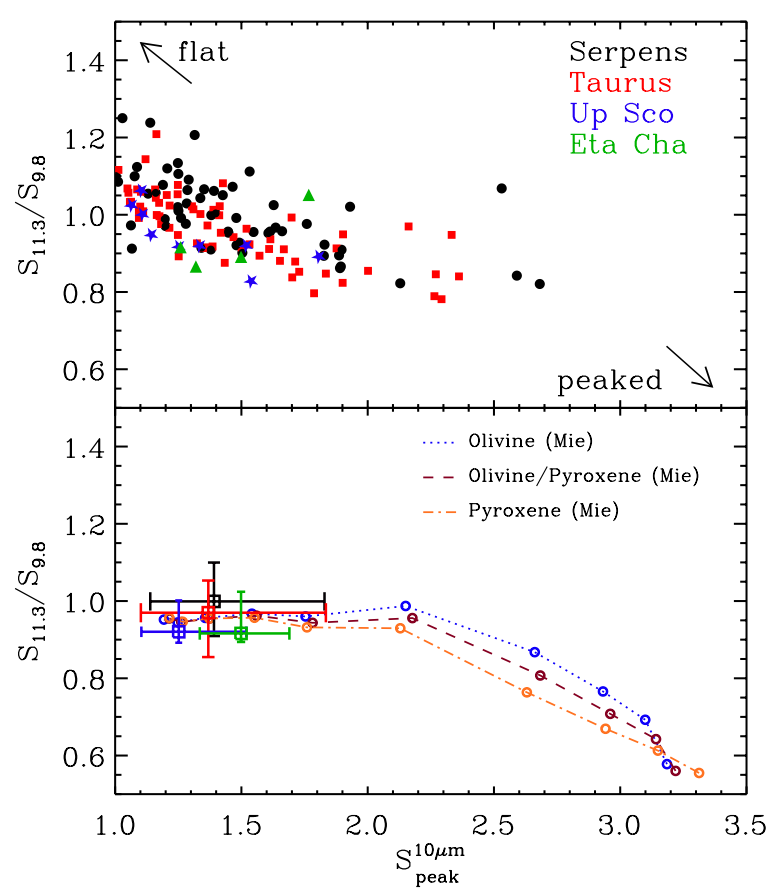

Figure 9. Top: the ratio of normalized fluxes at $11.3-9.8 \mu \mathrm{m}\left(S_{11.3} / S_{9.8}\right)$ is plotted against the peak at $10 \mu \mathrm{m}\left(S_{\text {peak }}^{10 \mu \mathrm{m}}\right)$ for Serpens (black dots), Taurus (red squares), Upper Scorpius (blue stars), and $\eta$ Chamaeleontis (green triangles). Bottom: squares show the median values and crosses indicate the 15-85 percentile ranges of the distributions (top panel). Colored curves are derived from theoretical opacities for different mixtures by Olofsson et al. (2009). The open circles correspond to different grain sizes, from left to right $6.25,5.2,4.3$, $3.25,2.7,2.0,1.5,1.25,1.0$, and $0.1 \mu \mathrm{m}$.

(A color version of this figure is available in the online journal.)

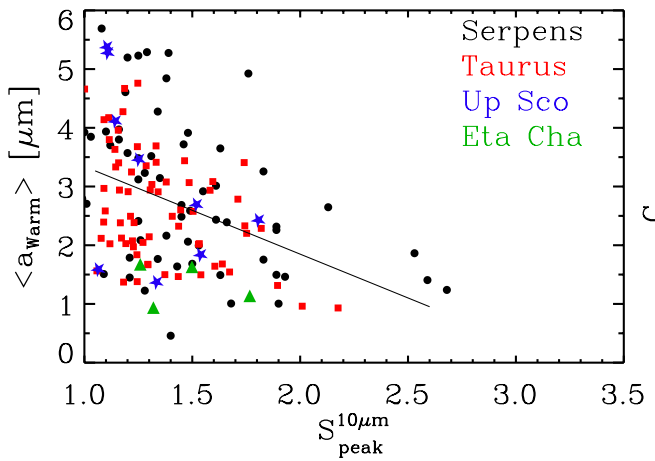

feature. These results argue against the results of Sargent et al. (2009) that find a high crystallinity fraction and small grains fitting low strengths of the $10 \mu \mathrm{m}$ silicate feature. Although it may be possible to fit a few spectra with a certain prescription, a good model should be able to explain the robust relationship between the strength and shape of the $10 \mu \mathrm{m}$ silicate feature observed for large numbers of disks. Despite the many processes able to change the shape or the strength of this feature, only grain size has so far demonstrated the capability to explain the observed trend. Our conclusion is that $S_{\mathrm{peak}}^{10 \mu \mathrm{m}}$ and dust sizes are appropriately correlated.

\subsection{Comparison with Other Studies}

Dust composition results are available in the literature for the disks in Taurus and $\eta$ Cha (see Table 4 for an overview). SiciliaAguilar et al. (2009) present their analysis in $\eta$ Cha considering the same five dust species and three grain sizes (enstatite in their model is the only species for which only the two smaller grain sizes are considered), but for a distribution of temperatures derived using the Two Layer Temperature Distribution (TLTD; Juhász et al. 2009) decomposition procedure. For the same four objects, their mean amorphous fraction is $80.1 \% \pm 9.3 \%$. This result is consistent with the $82.8 \% \pm 12.9 \%$ mean amorphous fraction found here. The mean crystalline fractions derived are $18.4 \% \pm 10.7 \%$ with TLTD and $17.1 \% \pm 12.8 \%$ derived here.

Sargent et al. (2009) present their decomposition procedure for 65 YSOs in Taurus. This method also takes into consideration a warm and a cold temperature, and makes use of two amorphous species (olivine and pyroxene) with two grain sizes (small and large), and three crystalline species (enstatite, forsterite, and crystalline silica) of a single size. Their mean warm amorphous fraction is $82.9 \% \pm 19.3 \%$ and warm crystalline

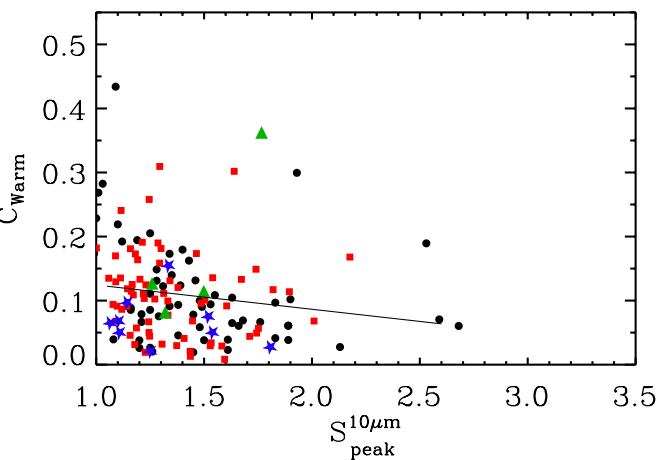

Figure 10. Left panel: strength of the $10 \mu \mathrm{m}$ silicate feature $\left(S_{\text {peak }}^{10 \mu \mathrm{m}}\right)$ vs. the mass-averaged mean grain size for the warm component. Right panel: strength of the $10 \mu \mathrm{m}$ silicate feature vs. crystalline fraction for the warm component. The best-fit relationships are shown for reference.

(A color version of this figure is available in the online journal.)

Table 4

Comparison of Mean Mineralogical Results from this Study With Literature Studies

\begin{tabular}{|c|c|c|c|c|}
\hline \multirow[t]{2}{*}{ Region } & \multicolumn{2}{|c|}{ This Work } & \multicolumn{2}{|c|}{ Literature } \\
\hline & Amorphous & Crystalline & Amorphous & Crystalline \\
\hline \multicolumn{5}{|c|}{ Warm component } \\
\hline Taurus & $89.0 \% \pm 6.6 \%$ & $10.9 \% \pm 6.6 \%$ & $82.9 \% \pm 19.3 \%$ & $17.1 \% \pm 19.3 \%^{\mathrm{a}}$ \\
\hline$\eta$ Cha & $82.8 \% \pm 12.9 \%$ & $17.1 \% \pm 12.8 \%$ & $80.1 \% \pm 9.3 \%$ & $18.4 \% \pm 10.7 \%^{b}$ \\
\hline \multicolumn{5}{|c|}{ Cold component } \\
\hline Taurus & $85.9 \% \pm 10.6 \%$ & $13.9 \% \pm 10.5 \%$ & $77.3 \% \pm 19.9 \%$ & $22.6 \% \pm 19.9 \%^{\mathrm{a}}$ \\
\hline
\end{tabular}

Notes.

${ }^{\text {a }}$ Sargent et al. (2009).

${ }^{\mathrm{b}}$ Sicilia-Aguilar et al. (2009). 


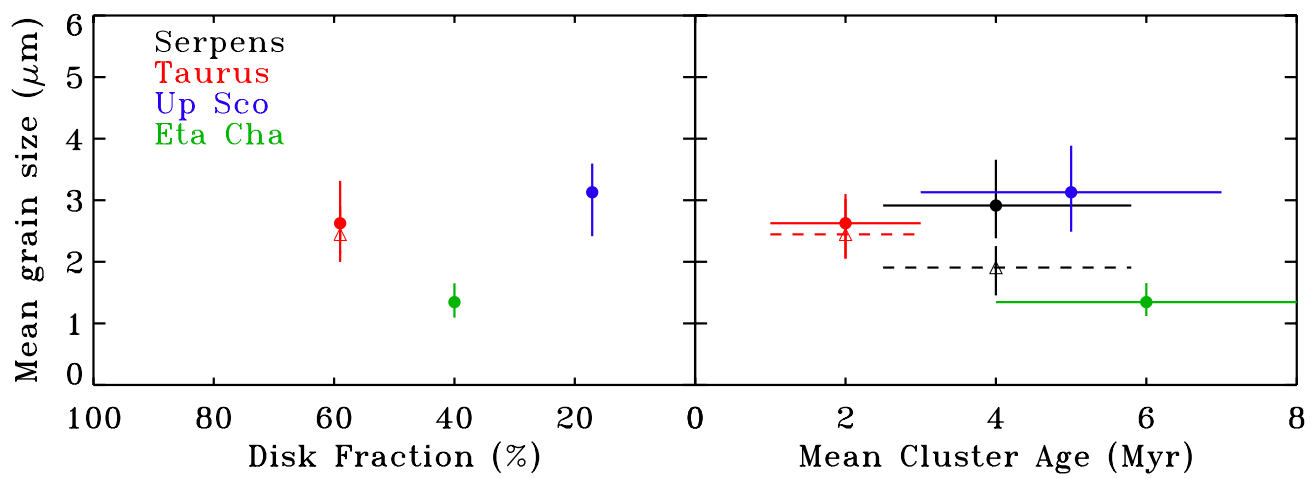

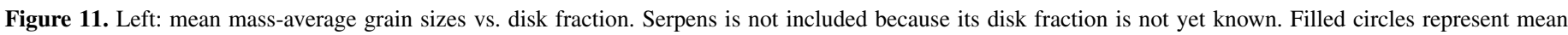

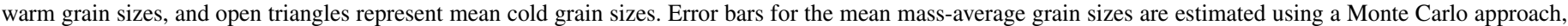

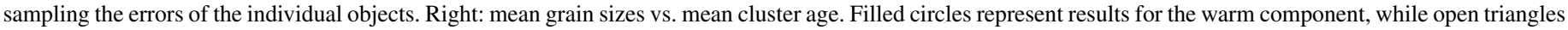
represent the cold component. The black points are YSOs in Serpens, red in Taurus, blue in Upper Sco, and green in $\eta$ Cha.

(A color version of this figure is available in the online journal.)

fraction is $17.1 \% \pm 19.3 \%$, while here the derived fractions are $89.0 \% \pm 6.6 \%$ and $10.9 \% \pm 6.6 \%$ for the warm amorphous and crystalline fractions, respectively. For the cold component, Sargent et al. (2009) derive a mean cold amorphous fraction of $77.3 \% \pm 19.9 \%$ and cold crystalline fraction of $22.6 \% \pm$ $19.9 \%$, while here the values are $85.9 \% \pm 10.6 \%$ and $13.9 \% \pm$ $10.5 \%$, respectively. The consistently lower amorphous (higher crystalline) fractions found by Sargent et al. (2009) could be a result of their choice to use silica in crystalline rather than amorphous form (as used here).

\section{DISCUSSION}

\subsection{Dust Characteristics}

Section 4 has shown that the disk populations in the four regions presented here, young and older, have very similar distributions in the two main dust parameters: grain size and composition. The large number of objects in the two young regions studied occupy a region in parameter space of either grain size or crystallinity fraction that is also populated by the small number of older disks. The grain sizes derived for the cold component never reach the largest grain size modeled $(6 \mu \mathrm{m})$, differing from the warm component results which span the entire range in sizes. The crystallinity fraction does not seem to be correlated with mean grain size, warm or cold. Whatever processes are responsible for the crystallization of the initially amorphous grains, they should not only be independent from the processes that govern the grain size distribution, but they should also be able to work on larger amorphous grains. Alternatively, the crystalline lattice should be able to keep itself regular during the coagulation of small crystalline dust to create big crystalline grains. The correlation between the strength of the $10 \mu \mathrm{m}$ feature and the mean grain size in disk surfaces, combined with the lack of correlation between crystallinity fraction and $S_{\text {peak }}^{10 \mu \mathrm{m}}$, supports the wide usage of $S_{\text {peak }}^{10 \mu \mathrm{m}}$ as a proxy for dust size in literature (van Boekel et al. 2003; Kessler-Silacci et al. 2006; Pascucci et al. 2009).

Bouwman et al. (2008) found a strong correlation between disk geometry and the strength of the $10 \mu \mathrm{m}$ silicate feature for a very small sample of $\mathrm{T}$ Tauri stars (seven disks), which points to flatter disks having shallower $10 \mu \mathrm{m}$ features (i.e., large grains in the disk surface). Using results from similar decomposition procedures, Olofsson et al. (2010) and Juhász et al. (2010) confirm this trend for larger samples of T Tauri
(58 disks) and Herbig Ae/Be stars (45 disks), respectively. Those trends are much weaker than that found by Bouwman et al. (2008), showing a larger spread. For the current even larger sample (139 disks), no significant trend is seen, indicating that the earlier small sample trends may have been affected by a few outliers. This result is similar to that found by Oliveira et al. (2010) for a large YSO sample ( 200 objects) using the strength of the $10 \mu \mathrm{m}$ silicate feature as a proxy for grain size (Figure 14 in that paper). As discussed by Oliveira et al., the sedimentation models of Dullemond \& Dominik (2008) expect a strong correlation of larger grains in flatter disks that is not seen. This means that sedimentation alone cannot be responsible for the distribution of mean grain sizes in the upper layers of protoplanetary disks around T Tauri stars. Furthermore, the lack of correlation between crystallinity fraction and disk geometry is not in support of the results of Watson et al. (2009) and Sargent et al. (2009), who find a link between increasing crystallinity fraction and dust sedimentation.

As discussed in Oliveira et al. (2010) for Serpens and Taurus, and confirmed by the addition of considerably older samples, there is no clear difference in the mean grain sizes in the disk surfaces with mean cluster age, which can be seen in Figure 11. This evidence supports the discussion in that paper that the dust population observed in the disk surface cannot be a result of a progressive, monotonic change of state from small amorphous grains, to large, more crystalline grains, or "grain growth and processing." The fact that the distribution of grain sizes in the upper layers of disks does not change with cluster age implies that an equilibrium of the processes of dust growth and fragmentation must exist, which also supports the existence of small grains in disks that are millions of years old whereas dust growth is a rapid process (Weidenschilling 1980; Dullemond \& Dominik 2005). That small dust is still seen in disks in older regions like Upper Sco and $\eta$ Cha argues that this equilibrium of processes is maintained for millions of years, as long as the disks are optically thick, but independent of them having a flared or flatter geometry.

\subsection{Evolution of Crystallinity with Time?}

Literature studies of disk fractions of different YSO clusters with different mean ages show a trend of decreasing disk fraction, i.e., disks dissipating with time, over some few millions of years (Haisch et al. 2001; Hernández et al. 2008). This decrease is clearly confirmed by the lower fraction of disks 


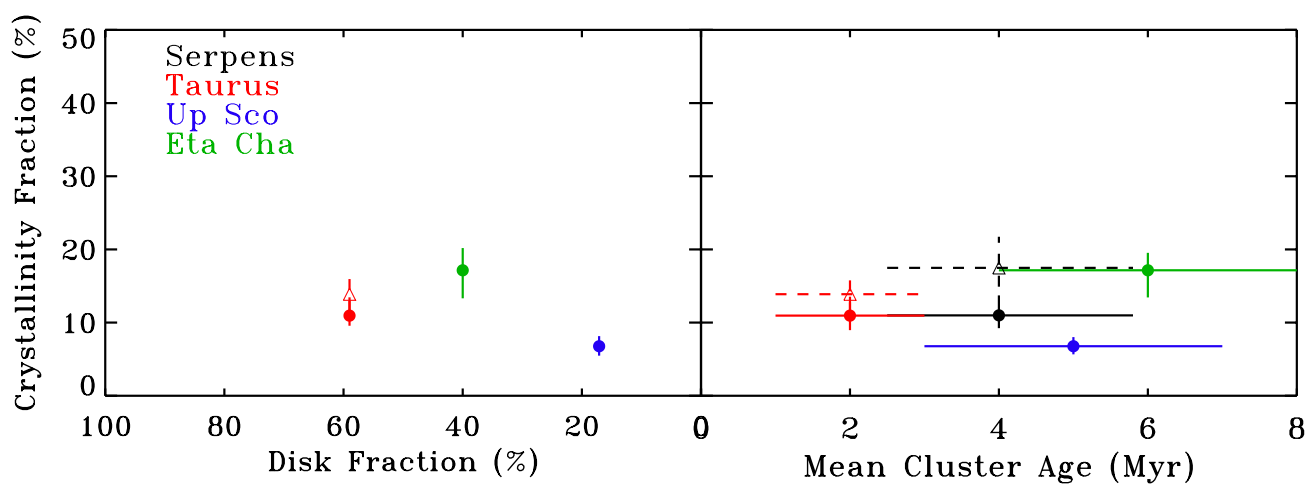

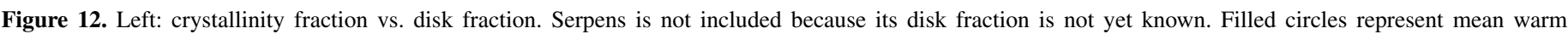

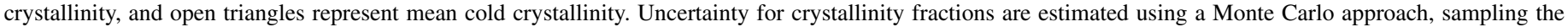

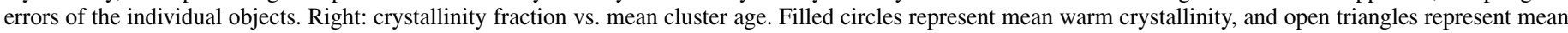
cold crystallinity. The black points are YSOs in Serpens, red in Taurus, blue in Upper Sco, and green in $\eta$ Cha.

(A color version of this figure is available in the online journal.)

still present in the older regions studied here (Upper Sco and $\eta$ Cha). According to current planet formation theories, if giant planets are to be formed from gas rich disks, the optically thin, gas-poor disks in those older regions should already harbor (proto-)planets. Considering the evidence from small bodies in our own solar system that suggest considerably higher crystallinity fractions than ISM dust (see Wooden et al. 2007 and Pontoppidan \& Brearley 2010 for reviews of the latest results), a crystallinity increase must occur.

In Figure 12, the mean crystallinity fraction per region is plotted against two evolutionary parameters: disk fraction (left) and mean age (right). Within the spread in individual fractions it is seen that, just as for grain sizes, there is no strong evidence of an increase of crystallinity fraction with either evolutionary parameter. This implies that there is no evolution in grain sizes or crystallinity fraction for the dust in the surface of disks over cluster ages in the range 1-8 Myr, as probed by the observations presented here. Essentially, there is no change in these two parameters until the disks disperse. Starting from the assumption that initially the dust in protoplanetary disks is of ISM origin (sub- $\mu \mathrm{m}$ in size and almost completely amorphous), it appears that a modest level of crystallinity is established in the disk surface early in the evolution ( $\leqslant 1 \mathrm{Myr}$ ) and then reaches some sort of steady state, irrespective of what is taking place in the disk midplane. Thus, the dust in the upper layers of disks does not seem to be a good tracer of the evolution that is taking place in the disk interior, where dust is growing further for the formation of planetesimals and planets, at many times higher crystallinity fractions, to be consistent with evidence from solar system bodies.

If this is the case, within $1 \mathrm{Myr}$ this surface dust must be crystallized to the observed fraction $(\sim 10 \%-20 \%)$. This result puts constraints on the formation of circumstellar disks. One possibility is that this crystallization of the dust in disks mostly occurs during the embedded phase. In this early stage of star formation, where large quantities of material are still accreting toward the protostar, a fraction of the infalling material comes very close to the protostar and is heated to temperatures $>800 \mathrm{~K}$ before it moves outward in the disk. Alternatively, accretion shocks or episodic heating events could be responsible for thermally annealing the dust in the disk surface.

The two-dimensional models of Visser \& Dullemond (2010) treat the radial evolution of crystals in time. According to these models, $100 \%$ of the dust in the inner disk $(\leqslant 1 \mathrm{AU})$ is crystallized within $1 \mathrm{Myr}$. With time, the inner disk crystalline fraction drops as the disk spreads, and crystalline material is transported to outer parts of the disk. These models can help explain the rapid crystallization required to account for our results. However, the models do show a decrease in inner disk $(\leqslant 1 \mathrm{AU})$ crystallinity fraction with time, which is not supported by our results. Since these models do not discriminate on vertical structure, but rather present crystallinity fractions that are integrated over all heights at a given radius, this decrease in crystallinity fraction is not necessarily connected to the surface of the disk. Thus, the decrease in crystallinity fraction with time found in the models of Visser \& Dullemond (2010) could be explained as a decrease in crystallinity fraction just in the disk midplane where the bulk of the mass resides, but not in the surface layers, as our data indicate. That would imply that radial mixing of these crystals is more efficient than vertical mixing, which is responsible for the crystallinity fraction decrease in the disk midplane.

According to the models of Ciesla (2007) for outward transport of high temperature materials, variations in radial transport dynamics with height produce vertical gradients in the crystalline fractions, such that the upper layers of the disk will have lower crystallinity fractions than the midplane population. If that is the case, the observations discussed here, which probe the disk surface only, lead to lower limits on the real crystalline fraction of disk midplanes. In this scenario, planets (and comets) forming in the disk midplane would have higher crystalline abundances than those derived here for the disk surfaces, which are compatible with what has been observed in our solar system. However, this model does not make predictions for the time evolution of the systems. Combination of the vertical and radial mixing processes with evolutionary models such as those of Visser \& Dullemond (2010) is needed to investigate whether older and younger disks could still show the same distribution of crystallinity fractions in the upper layers of disks, as observed here.

\section{CONCLUSIONS}

This paper presents the spectral decomposition of Spitzer/ IRS spectra using the B2C decomposition model of Olofsson et al. (2010). Mineralogical compositions and size distributions of dust grains in the surface layers of protoplanetary disks are 
Table 5

Dust Composition Derived Using the "B2C" Procedure

\begin{tabular}{|c|c|c|c|c|c|c|c|c|c|c|c|c|c|}
\hline \multirow[t]{2}{*}{ ID } & \multirow[t]{2}{*}{ SpT } & \multicolumn{4}{|c|}{$0.1(\mu \mathrm{m})$} & \multicolumn{4}{|c|}{$1.5(\mu \mathrm{m})$} & \multicolumn{4}{|c|}{$6.0(\mu \mathrm{m})$} \\
\hline & & Oli/Pyr ${ }^{\mathrm{b}}(\%)$ & Ens $(\%)$ & For $(\%)$ & Sil (\%) & Oli/Pyr (\%) & Ens $(\%)$ & For $(\%)$ & Sil (\%) & Oli/Pyr (\%) & Ens (\%) & For $(\%)$ & Sil (\%) \\
\hline \multicolumn{14}{|c|}{ Serpens } \\
\hline 1 & $\mathrm{~K} 2$ & $29.7_{-15.3}^{+10.7}$ & $1.0_{-0.4}^{+2.0}$ & $2.6_{-1.4}^{+2.6}$ & $0.1_{-0.0}^{+0.9}$ & $53.7_{-23.0}^{+16.5}$ & $3.5_{-2.4}^{+3.5}$ & $0.0_{-0.0}^{+2.9}$ & $1.0_{-0.0}^{+2.1}$ & $7.8_{-0.0}^{+3.8}$ & $\ldots$ & $\ldots$ & $0.6_{-0.0}^{+1.9}$ \\
\hline$\cdots$ & & $38.4_{-15.8}^{+14.5}$ & $0.0_{-0.0}^{+1.6}$ & $8.7_{-3.6}^{+5.5}$ & $1.2_{-0.0}^{+3.0}$ & $29.3_{-15.3}^{+6.2}$ & $6.8_{-2.8}^{+4.3}$ & $0.2_{-0.0}^{+1.1}$ & $0.3_{-0.0}^{+1.4}$ & $9.7_{-4.4}^{+4.7}$ & $\ldots$ & $\ldots$ & $5.5_{-1.6}^{+1.4}$ \\
\hline 3 & M0 & $26.5_{-7.3}^{+15.9}$ & $0.0_{-0.0}^{+2.4}$ & $0.9_{-0.4}^{+3.5}$ & $0.0_{-0.0}^{+1.7}$ & $43.9_{-18.6}^{+19.8}$ & $0.2_{-0.2}^{+4.1}$ & $2.7_{-2.1}^{+3.8}$ & $0.0_{-0.0}^{+2.9}$ & $15.7_{-2.8}^{+3.2}$ & $\ldots$ & $\ldots$ & $0.0_{-0.0}^{+1.0}$ \\
\hline$\ldots$ & & $27.3_{-8.6}^{+33.7}$ & $0.0_{-0.0}^{+10.5}$ & $10.2_{-4.9}^{+19.1}$ & $0.0_{-4.0}^{+16.3}$ & $32.9_{-9.9}^{+34.1}$ & $4.9_{-5.5}^{+19.2}$ & $0.0_{-2.9}^{+13.9}$ & $12.7_{-5.6}^{+22.5}$ & $10.2_{-4.7}^{+7.4}$ & $\ldots$ & $\ldots$ & $1.8_{-0.6}^{+2.2}$ \\
\hline 6 & K5 & $25.4_{-6.0}^{+4.5}$ & $0.0_{-0.0}^{+0.7}$ & $0.8_{-0.0}^{+1.1}$ & $0.0_{-0.0}^{+0.3}$ & $38.2_{-7.4}^{+11.6}$ & $1.0_{-0.0}^{+1.4}$ & $1.0_{-0.0}^{+1.4}$ & $0.0_{-0.0}^{+1.4}$ & $28.8_{-5.0}^{+4.5}$ & $\ldots$ & $\ldots$ & $4.8_{-1.8}^{+3.6}$ \\
\hline$\ldots$ & & $6.4_{-3.3}^{+3.8}$ & $4.1_{-2.3}^{+2.1}$ & $5.1_{-0.0}^{+3.4}$ & $0.5_{-0.0}^{+1.1}$ & $46.1_{-20.3}^{+19.8}$ & $4.7_{-0.0}^{+6.2}$ & $1.2_{-0.0}^{+2.3}$ & $2.7_{-0.0}^{+4.4}$ & $25.4_{-7.6}^{+8.6}$ & $\ldots$ & $\ldots$ & $3.7_{-0.0}^{+2.2}$ \\
\hline 7 & M0 & $3.7_{-0.9}^{+3.1}$ & $0.0_{-0.0}^{+2.9}$ & $0.8_{-0.0}^{+4.3}$ & $0.0_{-0.0}^{+0.9}$ & $75.6_{-23.9}^{+26.0}$ & $0.0_{-0.1}^{+5.6}$ & $3.7_{-3.0}^{+4.7}$ & $0.0_{-0.0}^{+5.0}$ & $0.0_{-0.0}^{+9.8}$ & $\ldots$ & $\ldots$ & $16.1_{-13.7}^{+4.4}$ \\
\hline$\ldots$ & & $0.0_{-0.5}^{+3.4}$ & $7.2_{-6.9}^{+4.9}$ & $8.1_{-6.9}^{+5.3}$ & $14.1_{-11.5}^{+8.3}$ & $6.0_{-60}^{+7.7}$ & $14.1_{-11.7}^{+8.8}$ & $0.0_{-0.1}^{+3.0}$ & $24.7_{-19.7}^{+14.3}$ & $25.6_{-13.9}^{+14.0}$ & $\ldots$ & $\cdots$ & $0.0_{-2.6}^{+3.5}$ \\
\hline 9 & $\ldots$ & $58.3_{-8.8}^{+10.9}$ & $0.0_{-0.0}^{+1.4}$ & $0.5_{-0.0}^{+1.1}$ & $0.0_{-0.0}^{+0.7}$ & $27.4_{-7.2}^{+5.7}$ & $3.8_{-2.1}^{+2.4}$ & $2.5_{-2.0}^{+1.5}$ & $0.0_{-0.0}^{+1.3}$ & $3.6_{-0.0}^{+4.1}$ & $\cdots$ & $\cdots$ & $3.7_{-0.0}^{+1.7}$ \\
\hline$\cdots$ & & $24.5_{-14.5}^{+16.0}$ & $0.0_{-0.0}^{+3.5}$ & $14.4_{-9.8}^{+13.9}$ & $16.9_{-15.0}^{+24.0}$ & $9.4_{-6.1}^{+6.7}$ & $0.0_{-0.8}^{+3.8}$ & $0.0_{-0.0}^{+1.5}$ & $\begin{array}{l}7.5_{-5.2}^{+5.8} \\
\end{array}$ & $24.4_{-14.3}^{+16.2}$ & $\ldots$ & $\ldots$ & $2.7_{-3.2}^{+7.2}$ \\
\hline 10 & $\ldots$ & $1.9_{-0.6}^{+5.9}$ & $0.0_{-0.0}^{+2.5}$ & $2.3_{-0.7}^{+2.1}$ & $4.3_{-1.4}^{+2.4}$ & $37.6_{-16.8}^{+17.3}$ & $7.2_{-3.5}^{+4.2}$ & $5.3_{-2.9}^{+6.6}$ & $0.2_{-0.0}^{+4.0}$ & $31.6_{-8.3}^{+18.5}$ & $\ldots$ & $\ldots$ & $9.5_{-4.4}^{+7.6}$ \\
\hline$\ldots$ & & $37.4_{-9.4}^{+29.1}$ & $2.0_{-0.0}^{+10.1}$ & $0.0_{-0.0}^{+9.2}$ & $0.0_{-0.0}^{+19.4}$ & $39.0_{-10.3}^{+23.6}$ & $0.0_{-0.0}^{+20.1}$ & $3.3_{-1.3}^{+9.6}$ & $0.0_{-0.0}^{+9.3}$ & $14.8_{-1.2}^{+35.1}$ & $\ldots$ & $\ldots$ & $3.6_{-0.5}^{+14.1}$ \\
\hline 14 & M2 & $11.1_{-0.0}^{+46.8}$ & $0.0_{-0.0}^{+6.0}$ & $3.0_{-00}^{+7.1}$ & $0.0_{-0.0}^{+6.0}$ & $54.7_{-203}^{+31.3}$ & $4.8_{-0.0}^{+20.8}$ & $0.0_{-0.0}^{+4.9}$ & $0.0_{-0.0}^{+8.7}$ & $13.5_{-58}^{+9.3}$ & $\ldots$ & $\ldots$ & $12.8_{-40}^{+10.4}$ \\
\hline 15 & $\ldots$ & $35.6_{-7.0}^{+12.5}$ & $0.2_{-0.0}^{+1.5}$ & $2.9_{-1.3}^{+1.6}$ & $0.0_{-0.0}^{+0.6}$ & $51.3_{-16.1}^{+9.3}$ & $3.0_{-1.5}^{+3.4}$ & $0.0_{-0.0}^{+1.6}$ & $0.8_{-0.0}^{+1.8}$ & $1.2_{-0.0}^{+2.0}$ & $\ldots$ & $\ldots$ & $5.0_{-0.5}^{+2.0}$ \\
\hline$\ldots$ & & $36.3_{-12.9}^{+10.4}$ & $2.4_{-1.6}^{+1.8}$ & $2.2_{-1.0}^{+3.5}$ & $1.1_{-0.9}^{+2.8}$ & $34.9_{-12.2}^{+9.9}$ & $0.0_{-0.0}^{+1.0}$ & $5.4_{-2.1}^{+2.0}$ & $0.0_{-0.6}^{+2.1}$ & $14.6_{-4.6}^{+4.7}$ & $\ldots$ & $\ldots$ & $3.0_{-1.9}^{+1.4}$ \\
\hline 21 & $\ldots$ & $2.2_{-0.0}^{+5.0}$ & $0.0_{-0.0}^{+1.6}$ & $2.7_{-0.0}^{+3.7}$ & $0.1_{-0.0}^{+3.6}$ & $20.2_{-12.9}^{+10.1}$ & $0.1_{-0.0}^{+4.2}$ & $19.2_{-9.1}^{+11.1}$ & $0.0_{-0.0}^{+4.2}$ & $43.3_{-21.3}^{+.6 .5}$ & $\ldots$ & $\ldots$ & $12.3_{-9.1}^{+8.6}$ \\
\hline$\ldots$ & & $33.0_{-20.7}^{+18.1}$ & $1.6_{-0.0}^{+5.2}$ & $0.0_{-0.0}^{+2.3}$ & $1.3_{-0.0}^{+11.9}$ & $42.5_{-24.9}^{+22.6}$ & $2.7_{-0.0}^{+14.8}$ & $0.5_{-0.0}^{+3.8}$ & $0.0_{-0.0}^{+14.2}$ & $9.6_{-3.3}^{+8.7}$ & $\ldots$ & $\ldots$ & $8.7_{-7.5}^{+5.2}$ \\
\hline 29 & M2 & $1.4_{-0.0}^{+6.4}$ & $0.0_{-0.0}^{+3.0}$ & $1.6_{-0.0}^{+7.0}$ & $2.7_{-0.6}^{+6.0}$ & $71.0_{-49}^{+26.0}$ & $0.0_{-0.0}^{+9.3}$ & $4.4_{-3.1}^{+9.4}$ & $0.0_{-0 .}^{+7.9}$ & $13.0_{-7}^{+6.4}$ & $\ldots$ & $\ldots$ & $5.6_{-3 .}^{+14.7}$ \\
\hline 30 & M1 & $0.0_{-0.0}^{+9.7}$ & $0.1_{-0.0}^{+4.4}$ & $3.4_{-1.2}^{+4.6}$ & $4.5_{-0.2}^{+6.8}$ & $12.5_{-6.4}^{+8.5}$ & $12.5_{-3.4}^{+8.1}$ & $1.3_{-0.9}^{+5.9}$ & $1.5_{-0.8}^{+5.0}$ & $64.2_{-47.0}^{+18.1}$ & $\ldots$ & $\ldots$ & $0.0_{-0.0}^{+21.4}$ \\
\hline$\ldots$ & & $10.3_{-13.8}^{+51.7}$ & $1.7_{-3.2}^{+15.4}$ & $5.5_{-4.3}^{+14.1}$ & $8.1_{-6.3}^{+21.6}$ & $8.0_{-19.4}^{+58.7}$ & $9.1_{-5.1}^{+14.9}$ & $29.6_{-24.3}^{+44.9}$ & $11.4_{-10.3}^{+37.5}$ & $14.0_{-10.3}^{+36.3}$ & $\ldots$ & $\ldots$ & $2.1_{-2.3}^{+9.6}$ \\
\hline 31 & $\ldots$ & $1.2_{-0.0}^{+0.8}$ & $0.0_{-0.0}^{+0.8}$ & $0.8_{-0.0}^{+0.5}$ & $0.0_{-0.0}^{+1.0}$ & $15.0_{-1.5}^{+2.6}$ & $2.5_{-0.0}^{+1.2}$ & $3.4_{-1.3}^{+1.5}$ & $0.4_{-0.0}^{+0.4}$ & $76.1_{-5.4}^{+7.8}$ & $\ldots$ & $\cdots$ & $0.6_{-0.0}^{+1.0}$ \\
\hline$\ldots$ & & $21.8_{-4.9}^{+5.3}$ & $2.4_{-0.0}^{+1.6}$ & $0.0_{-0.0}^{+0.7}$ & $16.7_{-7.9}^{+8.7}$ & $24.0_{-4.0}^{+4.7}$ & $2.3_{-0.0}^{+2.0}$ & $0.5_{-0.0}^{+1.7}$ & $5.4_{-2.6}^{+4.4}$ & $9.2_{-5.2}^{+4.8}$ & $\ldots$ & $\ldots$ & $17.6_{-4.7}^{+3.3}$ \\
\hline 36 & K5 & $22.3_{-6.2}^{+5.2}$ & $0.9_{-0.0}^{+1.6}$ & $0.6_{-0.0}^{+1.3}$ & $0.0_{-0.0}^{+0.8}$ & $51.9_{-12.3}^{+12.1}$ & $1.0_{-0.6}^{+2.9}$ & $3.3_{-2.5}^{+2.8}$ & $0.0_{-0.0}^{+1.6}$ & $10.8_{-2.6}^{+2.8}$ & $\ldots$ & $\ldots$ & $9.0_{-4.8}^{+2.7}$ \\
\hline$\ldots$ & & $37.4_{-15.4}^{+11.0}$ & $0.0_{-0.0}^{+3.9}$ & $1.6_{-1.0}^{+2.7}$ & $2.9_{-2.5}^{+8.6}$ & $38.1_{-14.3}^{+11.0}$ & $0.0_{-0.0}^{+2.4}$ & $0.0_{-0.0}^{+2.9}$ & $0.2_{-0.0}^{+8.7}$ & $19.6_{-7.7}^{+5.9}$ & $\ldots$ & $\ldots$ & $0.1_{-1.4}^{+0.3}$ \\
\hline 40 & M7 & $0.0_{-0.0}^{+8.3}$ & $0.0_{-0.0}^{+8.4}$ & $2.9_{-0.8}^{+4.4}$ & $10.9_{-4.1}^{+4.6}$ & $41.2_{-25.0}^{+17.3}$ & $22.5_{-8.8}^{+11.0}$ & $17.9_{-7.4}^{+11.6}$ & $0.0_{-0.0}^{+5.5}$ & $4.5_{-0.2}^{+9.7}$ & $\ldots$ & $\ldots$ & $0.0_{-0.0}^{+9.8}$ \\
\hline$\ldots$ & & $24.2_{-18.3}^{+15.5}$ & $16.6_{-8.4}^{+11.2}$ & $3.1_{-0.0}^{+16.4}$ & $0.0_{-0.0}^{+4.8}$ & $18.2_{-13.8}^{+9.5}$ & $2.1_{-1.9}^{+12.8}$ & $13.4_{-9.8}^{+7.8}$ & $0.0_{-0.0}^{+3.0}$ & $0.2_{-0.0}^{+9.7}$ & $\ldots$ & $\ldots$ & $22.3_{-12.6}^{+11.2}$ \\
\hline 41 & $\mathrm{~K} 2$ & $0.0_{-0.0}^{+18.9}$ & $6.3_{-0.0}^{+19.1}$ & $3.9_{-0.0}^{+16.0}$ & $0.8_{-0.0}^{+8.6}$ & $66.6_{-582}^{+6.8}$ & $3.4_{-0.0}^{+16.3}$ & $16.4_{-8.6}^{+1.3}$ & $0.0_{-0.0}^{+6.3}$ & $2.6_{-0.0}^{+13.8}$ & $\ldots$ & $\ldots$ & $0.0_{-00}^{+14.8}$ \\
\hline 43 & M0.5 & $0.0_{-0.0}^{+13.0}$ & $0.0_{-0.0}^{+4.0}$ & $4.2_{-0.0}^{+5.7}$ & $4.6_{-0.0}^{+6.1}$ & $42.1_{-27.0}^{+19.1}$ & $7.1_{-5.1}^{+8.9}$ & $2.7_{-2.0}^{+7.1}$ & $0.0_{-0.0}^{+3.5}$ & $37.2_{-19.3}^{+15.8}$ & $\ldots$ & $\ldots$ & $2.0_{-0.0}^{+12.7}$ \\
\hline 48 & M5.5 & $7.0_{-0.1}^{+17.5}$ & $0.1_{-0.0}^{+8.4}$ & $2.0_{-0.0}^{+10.2}$ & $0.6_{-0.6}^{+3.0}$ & $73.3_{-35.2}^{+28.2}$ & $0.0_{-0.0}^{+9.2}$ & $0.0_{-0.1}^{+13.8}$ & $1.1_{-0.7}^{+9.9}$ & $6.0_{-2.1}^{+8.4}$ & $\ldots$ & $\ldots$ & $9.9_{-6.9}^{+4.6}$ \\
\hline$\cdots$ & & $46.0_{-40.6}^{+23.9}$ & $5.1_{-3.3}^{+10.9}$ & $2.5_{-1.1}^{+10.5}$ & $0.3_{-2.3}^{+21.3}$ & $18.7_{-17.6}^{+10.0}$ & $0.0_{-0.1}^{+7.5}$ & $4.1_{-3.5}^{+12.0}$ & $8.6_{-8.1}^{+5.8}$ & $7.4_{-4.7}^{+5.6}$ & $\ldots$ & $\ldots$ & $7.5_{-6.6}^{+4.4}$ \\
\hline 53 & M2.5 & $0.0_{-0.0}^{+36.7}$ & $5.0_{-0.0}^{+26.6}$ & $5.4_{-0.0}^{+18.5}$ & $5.9_{-0.0}^{+20.1}$ & $30.2_{-14.6}^{+18.2}$ & $10.1_{-5.8}^{+19.9}$ & $0.0_{-0.0}^{+11.6}$ & $2.3_{-0.7}^{+15.6}$ & $32.9_{-6.7}^{+27.1}$ & $\ldots$ & . & $8.1_{-5.3}^{+9.4}$ \\
\hline 55 & $\mathrm{~K} 2$ & $16.9_{-4.0}^{+11.3}$ & $1.6_{-0.0}^{+2.7}$ & $2.3_{-0.9}^{+2.5}$ & $0.0_{-0.0}^{+1.1}$ & $71.0_{-26.2}^{+16.2}$ & $0.0_{-0.0}^{+3.0}$ & $0.0_{-0.0}^{+2.7}$ & $1.9_{-0.0}^{+3.0}$ & $4.2_{-0.8}^{+1.5}$ & $\ldots$ & $\ldots$ & $2.1_{-0.7}^{+0.9}$ \\
\hline$\ldots$ & & $27.7_{-23.1}^{+81.3}$ & $4.8_{-1.1}^{+3.6}$ & $21.5_{-14.2}^{+34.7}$ & $0.0_{-0.0}^{+22.9}$ & $20.5_{-20.0}^{+71.1}$ & $15.7_{-9.9}^{+72.9}$ & $0.0_{-0.1}^{+22.3}$ & $4.8_{-4.2}^{+65.6}$ & $2.9_{-1.6}^{+5.8}$ & $\ldots$ & $\ldots$ & $1.9_{-1.8}^{+6.0}$ \\
\hline 56 & $\ldots$ & $7.8_{-2.0}^{+8.3}$ & $1.7_{-0.8}^{+1.9}$ & $2.5_{-0.6}^{+2.4}$ & $1.1_{-0.0}^{+3.4}$ & $44.6_{-28.5}^{+16.6}$ & $\begin{array}{l}5.0_{-2.7}^{+7.9} \\
\end{array}$ & $1.7_{-0.5}^{+7.3}$ & $0.0_{-0.0}^{+4.6}$ & $29.5_{-11.8}^{+11.8}$ & $\ldots$ & $\ldots$ & $6.1_{-0.1}^{+12.2}$ \\
\hline$\ldots$ & & $44.4_{-170}^{+22.2}$ & $1.6_{-10}^{+5.1}$ & $0.0_{-0.0}^{+6.0}$ & $2.2_{-0.0}^{+21.1}$ & $29.8_{-120}^{+11.0}$ & $0.0_{-0.0}^{+15.8}$ & $1.1_{-0.0}^{+7.5}$ & $0.0_{-0.0}^{+3.2}$ & $19.3_{-7.9}^{+24.2}$ & $\ldots$ & $\ldots$ & $1.6_{-0.0}^{+27.0}$ \\
\hline 57 & $\ldots$ & $2.2_{-0.0}^{+22.8}$ & $0.0_{-0.0}^{+9.1}$ & $5.7_{-1.1}^{+7.2}$ & $3.0_{-1.4}^{+3.2}$ & $72.0_{-40.9}^{+29.0}$ & $10.5_{-6.6}^{+13.3}$ & $0.0_{-0.0}^{+7.1}$ & $0.0_{-0.0}^{+7.1}$ & $6.5_{-0.0}^{+19.1}$ & $\ldots$ & $\ldots$ & $0.0_{-0.0}^{+9.5}$ \\
\hline$\ldots$ & & $23.0_{-5.8}^{+76.9}$ & $0.0_{-0.0}^{+79.5}$ & $14.4_{-10.4}^{+46.6}$ & $0.7_{-0.0}^{+131.2}$ & $13.1_{-4.8}^{+55.6}$ & $0.3_{-0.0}^{+27.7}$ & $4.5_{-0.0}^{+61.3}$ & $1.7_{-4.5}^{+25.5}$ & $25.1_{-3.7}^{+85.7}$ & $\ldots$ & $\ldots$ & $17.2_{-5.0}^{+81.4}$ \\
\hline 58 & K7 & $6.6_{-0.5}^{+3.2}$ & $0.0_{-0.0}^{+1.0}$ & $0.6_{-0.0}^{+1.1}$ & $1.2_{-0.0}^{+0.7}$ & $29.9_{-6.7}^{+4.6}$ & $1.0_{-0.7}^{+2.2}$ & $7.1_{-2.1}^{+3.0}$ & $0.0_{-0.0}^{+1.8}$ & $28.3_{-11.5}^{+6.5}$ & $\ldots$ & $\ldots$ & $25.4_{-8.7}^{+6.4}$ \\
\hline$\ldots$ & & $32.2_{-16.4}^{+16.8}$ & $3.2_{-0.0}^{+15.6}$ & $0.0_{-0.0}^{+3.7}$ & $0.0_{-0.0}^{+7.2}$ & $57.0_{-36.3}^{+24.9}$ & $0.0_{-0.0}^{+7.5}$ & $0.0_{-0.0}^{+8.0}$ & $0.0_{-0.0}^{+6.2}$ & $4.7_{-1.4}^{+2.9}$ & $\ldots$ & $\ldots$ & $2.8_{-0.0}^{+2.5}$ \\
\hline 60 & M0.5 & $0.0_{-0.1}^{+10.4}$ & $9.8_{-0.2}^{+23.0}$ & $5.2_{-0.0}^{+11.8}$ & $0.0_{-0.0}^{+3.0}$ & $41.7_{-21.7}^{+31.4}$ & $8.5_{-5.1}^{+13.0}$ & $3.3_{-2.3}^{+14.0}$ & $0.0_{-0.0}^{+13.0}$ & $4.9_{-1.7}^{-1.4}$ & $\ldots$ & $\ldots$ & $26.5_{-146}^{+17.0}$ \\
\hline 61 & M0 & $12.2_{-0.0}^{+0.0}$ & $\begin{array}{c}-0.0 \\
0.0_{-0.0}^{+0.6}\end{array}$ & $1.8_{-0.0}^{+1.3}$ & $0.0_{-0.0}^{+1.0}$ & $52.1_{-13.2}^{+11.9}$ & $3.1_{-0.0}^{+5.1}$ & $6.2_{-3.0}^{+3.6}$ & $0.0_{-0.0}^{+2.4}$ & $11.3_{-3.0}^{+4.1}$ & $\ldots$ & $\ldots$ & $13.3_{-6.3}^{+1.8}$ \\
\hline$\ldots$ & & $38.2_{-7.6}^{+3.7}$ & $0.0_{-0.0}^{+0.8}$ & $0.1_{-0.0}^{+0.9}$ & $0.6_{-0.0}^{+1.7}$ & $38.7_{-6.3}^{+6.6}$ & $0.0_{-0.0}^{+1.6}$ & $1.1_{-0.6}^{+0.9}$ & $1.2_{-0.1}^{+2.3}$ & $7.5_{-0.0}^{+5.7}$ & $\ldots$ & $\ldots$ & $12.7_{-2.4}^{+6.0}$ \\
\hline 65 & $\ldots$ & $0.0_{-0.0}^{+2.7}$ & $0.0_{-0.0}^{+2.0}$ & $6.0_{-2.5}^{+2.6}$ & $0.0_{-0.0}^{+1.0}$ & $21.3_{-16.6}^{+9.9}$ & $8.6_{-5.4}^{+5.3}$ & $8.3_{-3.2}^{+4.2}$ & $0.0_{-2.7}^{+1.3}$ & $41.8_{-22.4}^{+15.0}$ & $\ldots$ & $\ldots$ & $13.9_{-10.4}^{+7.9}$ \\
\hline$\ldots$ & & $54.9_{-19.1}^{+56.6}$ & $0.1_{-0.0}^{+7.9}$ & $0.0_{-0.0}^{+6.3}$ & $8.8_{-6.6}^{+16.2}$ & $6.2_{-2.8}^{+9.7}$ & $0.3_{-0.0}^{+9.6}$ & $0.0_{-0.0}^{+4.0}$ & $2.7_{-1.4}^{+3.8}$ & $8.5_{-2.5}^{+28.2}$ & $\ldots$ & $\ldots$ & $18.4_{-7.7}^{+20.9}$ \\
\hline 66 & $\mathrm{~K} 5$ & $2.1_{-0.0}^{+8.1}$ & $0.0_{-0.0}^{+1.6}$ & $0.1_{-0.0}^{+1.5}$ & $0.8_{-0.0}^{+2.4}$ & $46.5_{-15.6}^{+15.4}$ & $0.8_{-0.0}^{+5.1}$ & $2.9_{-1.6}^{+5.0}$ & $0.0_{-0.0}^{+2.3}$ & $38.2_{-18.7}^{+18.7}$ & $\ldots$ & $\ldots$ & $8.7_{-2.5}^{+8.2}$ \\
\hline$\ldots$ & & $\begin{array}{c}-0.0 \\
33.5_{-16.5}^{+10.5}\end{array}$ & $\begin{array}{l}-0.0 \\
3.9_{-0.0}^{+16.1}\end{array}$ & $\begin{array}{l}-0.0 \\
4.2_{-0.0}^{+14.3}\end{array}$ & $\begin{array}{l}-0.0 \\
0.0_{-0.0}^{+4.9}\end{array}$ & $\begin{array}{l}26.3_{-8.9}^{+25.6} \\
\end{array}$ & $1.7_{-0.0}^{+11.2}$ & $0.4_{-0.0}^{+8.1}$ & $3.2_{-0.0}^{+0.0}$ & $11.0_{-0.0}^{+21.0}$ & $\ldots$ & $\ldots$ & $\begin{array}{r}-2.5 \\
15.7_{-8.1}^{+6.8}\end{array}$ \\
\hline 71 & M3 & $6.4_{-0.0}^{+28.2}$ & $0.0_{-0.0}^{+1.8}$ & $2.3_{-0.0}^{+6.8}$ & $0.8_{-0.0}^{+8.7}$ & $24.2_{-11.4}^{+24.6}$ & $3.0_{-0.3}^{+20.1}$ & $4.7_{-1.6}^{+25.9}$ & $1.9_{-0.5}^{+12.1}$ & $56.6_{-10.9}^{+55.6}$ & $\ldots$ & $\ldots$ & $0.0_{-0.0}^{+10.4}$ \\
\hline 74 & $\ldots$ & $0.0_{-0.0}^{+5.0}$ & $0.0_{-0.0}^{+0.0}$ & $6.3_{-2.8}^{+3.7}$ & $13.4_{-6.3}^{+4.8}$ & $\begin{array}{l}0_{-0.0}^{+6.34} \\
+6.3\end{array}$ & $\begin{array}{r}-0.3 \\
5.8_{-3.0}^{+3.4}\end{array}$ & $16.1_{-10.0}^{+5.0}$ & $0.0_{-0.0}^{+4.7}$ & $58.3_{-31.2}^{+17.9}$ & $\ldots$ & $\ldots$ & $0.0_{-0.0}^{+18.2}$ \\
\hline$\ldots$ & & $50.7_{-195}^{+33.8}$ & $0.1_{-0.0}^{+11.1}$ & $0.1_{-0.0}^{+6.6}$ & $0.0_{-0.0}^{+29.4}$ & $24.2_{-9.8}^{+26.5}$ & $1.8_{-0.0}^{+23.4}$ & $5.4_{-0.0}^{+31.1}$ & $0.0_{-0.0}^{+12.8}$ & $9.9_{-5.5}^{+22.5}$ & $\ldots$ & $\ldots$ & $7.8_{-7.1}^{+23.4}$ \\
\hline 75 & $\ldots$ & $0.0_{-0.0}^{+72.5}$ & $0.0_{-0.0}^{+10.2}$ & $4.1_{-0.0}^{+28.5}$ & $0.1_{-0.0}^{+20.2}$ & $15.0_{-0.0}^{+>15.0}$ & $5.2_{-0.0}^{+36.0}$ & $0.0_{-0.0}^{+32.5}$ & $0.0_{-0.0}^{+51.2}$ & $59.6_{-33.4}^{+42.6}$ & $\ldots$ & $\ldots$ & $16.0_{-0.0}^{+69.6}$ \\
\hline 76 & M1 & $5.3_{-3.4}^{+9.6}$ & $0.0_{-0.0}^{+2.8}$ & $2.6_{-0.0}^{+3.3}$ & $1.9_{-0.0}^{+3.0}$ & $65.0_{-29.6}^{+15.7}$ & $0.0_{-0.0}^{+3.3}$ & $1.3_{-0.0}^{+5.3}$ & $0.0_{-0.0}^{+3.0}$ & $23.7_{-3.9}^{+13.5}$ & . & $\ldots$ & $0.1_{-0.0}^{+2.0}$ \\
\hline$\ldots$ & & $41.8_{-23.6}^{+17.5}$ & $3.6_{-1.9}^{+0.5}$ & $0.0_{-0.0}^{+3.3 .}$ & $0.0_{-0.0}^{+2.8}$ & $29.6_{-12.1}^{+12.4}$ & $0.0_{-0.0}^{+3.3}$ & $12.9_{-5.2}^{+7.0}$ & $0.0_{-0.0}^{+2.0}$ & $12.2_{-4.3}^{+5.9}$ & $\ldots$ & $\ldots$ & $0.0_{-1.0}^{+2.1}$ \\
\hline
\end{tabular}


Table 5

(Continued)

\begin{tabular}{|c|c|c|c|c|c|c|c|c|c|c|c|c|c|}
\hline \multirow[t]{2}{*}{ ID } & \multirow[t]{2}{*}{ SpT } & \multicolumn{4}{|c|}{$0.1(\mu \mathrm{m})$} & \multicolumn{4}{|c|}{$1.5(\mu \mathrm{m})$} & \multicolumn{4}{|c|}{$6.0(\mu \mathrm{m})$} \\
\hline & & Oli/Pyr ${ }^{\mathrm{b}}(\%)$ & Ens $(\%)$ & For $(\%)$ & Sil (\%) & Oli/Pyr (\%) & Ens $(\%)$ & For $(\%)$ & Sil (\%) & Oli/Pyr (\%) & Ens $(\%)$ & For $(\%)$ & Sil (\%) \\
\hline 80 & . & $0.3_{-0.0}^{+1.2}$ & $0.0_{-0.0}^{+0.2}$ & $1.2_{-0.0}^{+0.5}$ & $0.0_{-0.0}^{+0.4}$ & $7.5_{-0.0}^{+2.0}$ & $3.2_{-1.5}^{+1.3}$ & $3.1_{-0.9}^{+1.0}$ & $0.0_{-0.0}^{+0.6}$ & $84.7_{-6.6}^{+8.2}$ & $\ldots$ & $\cdots$ & $0.0_{-0.0}^{+1.1}$ \\
\hline$\ldots$ & & $6.4_{-00}^{+50.4}$ & $8.3_{-0.0}^{+29.8}$ & $0.6_{-0.0}^{+12.4}$ & $7.4_{-0.0}^{+34.2}$ & $15.4_{-0.2}^{+50.0}$ & $6.2_{-0.2}^{+18.2}$ & $0.8_{-0.1}^{+10.1}$ & $3.1_{-0.0}^{+9.0}$ & $22.6_{-0.0}^{+78.6}$ & $\cdots$ & .. & $29.2_{-0.0}^{+67.6}$ \\
\hline 81 & M5 & $10.7_{-0.0}^{+2.8}$ & $0.0_{-0.0}^{+0.8}$ & $0.0_{-0.0}^{+0.4}$ & $4.3_{-0.0}^{+1.2}$ & $54.5_{-3.7}^{+5.4}$ & $0.0_{-0.0}^{+0.7}$ & $6.1_{-2.0}^{+1.9}$ & $0.0_{-0.0}^{+0.5}$ & $15.6_{-2.7}^{+2.5}$ & $\cdots$ & $\ldots$ & $8.8_{-1.2}^{+1.6}$ \\
\hline$\cdots$ & & $45.4_{-5.1}^{+6.2}$ & $0.0_{-0.0}^{+1.1}$ & $0.0_{-0.0}^{+0.4}$ & $0.0_{-0.0}^{+1.5}$ & $31.4_{-4.4}^{+8.3}$ & $7.0_{-2.2}^{+2.4}$ & $0.0_{-0.0}^{+0.3}$ & $4.0_{-2.0}^{+2.3}$ & $7.7_{-1.2}^{+1.0}$ & $\ldots$ & $\ldots$ & $4.5_{-0.9}^{+0.7}$ \\
\hline 86 & M5.5 & $15.7_{-1.7}^{+3.1}$ & $0.0_{-0.0}^{+0.5}$ & $0.8_{-0.0}^{+0.7}$ & $0.0_{-0.0}^{+0.2}$ & $36.0_{-3.0}^{+3.4}$ & $0.0_{-0.0}^{+0.2}$ & $3.3_{-1.7}^{+1.1}$ & $0.0_{-0.0}^{+0.2}$ & $44.1_{-4.4}^{+4.2}$ & $\ldots$ & $\ldots$ & $0.1_{-0.0}^{+0.7}$ \\
\hline$\cdots$ & & $26.7_{-0.0}^{+83.1}$ & $12.2_{-0.0}^{+28.5}$ & $0.0_{-0.0}^{+38.4}$ & $1.9_{-0.0}^{+14.8}$ & $18.2_{-0.0}^{+55.5}$ & $10.0_{-0.0}^{+27.7}$ & $0.5_{-0.0}^{+20.3}$ & $6.0_{-0.0}^{+29.6}$ & $10.7_{-0.0}^{+39.6}$ & $\ldots$ & $\ldots$ & $13.7_{-0.0}^{+38.7}$ \\
\hline 88 & M0.5 & $0.0_{-0.0}^{+5.0}$ & $0.0_{-0.0}^{+1.0}$ & $8.6_{-0.0}^{+4.4}$ & $0.0_{-0.0}^{+0.4}$ & $67.1_{-17.3}^{+20.5}$ & $10.3_{-5.0}^{+7.1}$ & $0.0_{-0.0}^{+3.6}$ & $3.1_{-2.0}^{+2.4}$ & $9.9_{-1.8}^{+6.3}$ & $\ldots$ & $\ldots$ & $0.9_{-0.6}^{+1.2}$ \\
\hline$\ldots$ & & $6.0_{-10.0}^{+30.9}$ & $0.7_{-9.0}^{+37.8}$ & $33.3_{-13.7}^{+22.2}$ & $6.0_{-9.2}^{+31.7}$ & $2.1_{-6.5}^{+21.3}$ & $0.0_{-0.0}^{+7.7}$ & $30.6_{-12.4}^{+29.1}$ & $0.6_{-3.0}^{+9.9}$ & $17.6_{-10.1}^{+32.8}$ & $\ldots$ & $\cdots$ & $3.1_{-3.5}^{+11.2}$ \\
\hline 89 & K5 & $0.0_{-0.0}^{+0.7}$ & $0.0_{-0.0}^{+0.2}$ & $2.6_{-1.0}^{+1.6}$ & $1.6_{-0.0}^{+0.7}$ & $0.0_{-0.0}^{+0.7}$ & $0.0_{-0.0}^{+0.6}$ & $1.4_{-0.0}^{+0.9}$ & $0.0_{-0.0}^{+0.6}$ & $94.4_{-75.4}^{+18.8}$ & $\cdots$ & $\ldots$ & $0.0_{-0.0}^{+3.7}$ \\
\hline 90 & $\ldots$ & $26.0_{-12.1}^{+20.9}$ & $0.0_{-0.1}^{+4.1}$ & $3.0_{-1.6}^{+4.4}$ & $0.0_{-0.0}^{+2.7}$ & $49.1_{-30.7}^{+24.7}$ & $3.7_{-1.8}^{+9.5}$ & $0.0_{-0.0}^{+10.1}$ & $2.8_{-1.9}^{+5.5}$ & $5.4_{-1.2}^{+16.4}$ & $\ldots$ & $\ldots$ & $10.0_{-1.8}^{+12.9}$ \\
\hline$\ldots$ & & $31.1_{-0.0}^{+65.4}$ & $7.5_{-1.4}^{+13.4}$ & $8.5_{-0.0}^{+51.3}$ & $0.7_{-0.0}^{+21.3}$ & $16.8_{-0.0}^{+54.1}$ & $3.6_{-0.0}^{+38.8}$ & $9.2_{-0.0}^{+58.7}$ & $0.8_{-0.0}^{+24.8}$ & $12.5_{-0.0}^{+52.2}$ & $\ldots$ & $\ldots$ & $9.2_{-0.0}^{+39.3}$ \\
\hline 92 & M0 & $1.1_{-0.0}^{+9.7}$ & $0.0_{-0.0}^{+0.9}$ & $4.8_{-0.0}^{+4.8}$ & $2.3_{-0.1}^{+5.3}$ & $35.8_{-8.5}^{+25.4}$ & $5.7_{-2.1}^{+7.5}$ & $0.0_{-0.0}^{+3.5}$ & $0.0_{-0.0}^{+2.4}$ & $44.0_{-15.9}^{+13.1}$ & $\ldots$ & $\ldots$ & $6.3_{-2.4}^{+14.1}$ \\
\hline$\ldots$ & & $19.6_{-7.1}^{+9.7}$ & $0.8_{-0.0}^{+3.4}$ & $0.2_{-0.0}^{+9.0}$ & $3.0_{-0.0}^{+4.6}$ & $31.2_{-151}^{+9.3}$ & $0.4_{-0.0}^{+6.1}$ & $6.8_{-3.9}^{+3.8}$ & $0.0_{-0.0}^{+10.0}$ & $37.9_{-12.3}^{+19.4}$ & $\ldots$ & $\ldots$ & $0.0_{-0.0}^{+12.6}$ \\
\hline 96 & M1 & $18.2_{-11.8}^{+8.7}$ & $2.6_{-2.0}^{+3.8}$ & $2.8_{-1.6}^{+4.6}$ & $0.0_{-0.0}^{+5.9}$ & $56.5_{-35.1}^{+23.3}$ & $3.8_{-1.8}^{+12.9}$ & $0.0_{-0.0}^{+15.2}$ & $3.0_{-0.8}^{+9.2}$ & $7.4_{-4.3}^{+6.7}$ & $\cdots$ & $\cdots$ & $5.8_{-1.5}^{+10.1}$ \\
\hline 100 & $\ldots$ & $0.0_{-0.0}^{+29.3}$ & $0.0_{-0.0}^{+12.0}$ & $2.8_{-0.0}^{+42.3}$ & $0.5_{-0.0}^{+35.5}$ & $1.2_{-0.0}^{+88.8}$ & $9.5_{-0.0}^{+81.9}$ & $0.0_{-0.0}^{+34.1}$ & $1.1_{-0.0}^{+106.2}$ & $81.8_{-54.1}^{+47.3}$ & $\ldots$ & $\ldots$ & $3.1_{-4.1}^{+11.6}$ \\
\hline 101 & $\ldots$ & $24.1_{-6.9}^{+26.6}$ & $0.0_{-0.0}^{+4.1}$ & $2.0_{-1.4}^{+3.2}$ & $1.0_{-0.0}^{+0.2}$ & $60.2_{-33.4}^{+22.6}$ & $1.9_{-0.0}^{+8.8}$ & $2.6_{-0.5}^{+7.5}$ & $0.0_{-0.0}^{+5.3}$ & $6.6_{-0.0}^{+30.1}$ & $\ldots$ & $\ldots$ & $1.6_{-0.0}^{+5.1}$ \\
\hline$\ldots$ & & $48.0_{-19.8}^{+7.1}$ & $0.0_{-0.1}^{+2.7}$ & $0.2_{-0.0}^{+2.7}$ & $2.5_{-1.9}^{+3.1}$ & $55.9_{-11.9}^{+7.1}$ & $0.0_{-0.0}^{+3.7}$ & $9.5_{-6.1}^{+4.8}$ & $0.0_{-0.0}^{+2.7}$ & $13.6_{-7.2}^{+3.5}$ & $\cdots$ & $\ldots$ & $0.1_{-0.0}^{+3.0}$ \\
\hline 103 & $\ldots$ & $0.0_{-0.0}^{+0.9}$ & $0.0_{-0.0}^{+0.3}$ & $2.4_{-0.0}^{+0.6}$ & $3.2_{-0.0}^{+0.9}$ & $38.0_{-5.0}^{+7.0}$ & $2.0_{-1.2}^{+2.3}$ & $7.8_{-2.1}^{+2.4}$ & $0.0_{-0.0}^{+1.8}$ & $27.6_{-5.5}^{+6.0}$ & $\ldots$ & $\ldots$ & $18.9_{-4.1}^{+4.2}$ \\
\hline$\ldots$ & & $11.0_{-9.1}^{+3.7}$ & $5.8_{-2.7}^{+4.2}$ & $0.0_{-0.0}^{+3.9}$ & $5.8_{-1.8}^{+3.3}$ & $35.1_{-20.5}^{+18.1}$ & $0.0_{-0.0}^{+6.3}$ & $5.1_{-0.0}^{+5.6}$ & $4.2_{-0.0}^{+9.0}$ & $15.7_{-4.5}^{+8.5}$ & $\ldots$ & $\ldots$ & $17.3_{-17.7}^{+2.3}$ \\
\hline 104 & $\ldots$ & $1.6_{-1.1}^{+14.7}$ & $0.9_{-0.2}^{+6.1}$ & $3.7_{-2.3}^{+5.2}$ & $4.3_{-1.8}^{+4.1}$ & $70.4_{-48.8}^{+35.4}$ & $4.7_{-3.6}^{+12.4}$ & $0.0_{-0.0}^{+10.2}$ & $0.0_{-0.0}^{+6.1}$ & $14.2_{-6.1}^{+17.3}$ & $\ldots$ & $\ldots$ & $0.1_{-0.0}^{+14.8}$ \\
\hline 106 & M3 & $0.0_{-0.0}^{+16.0}$ & $5.1_{-0.0}^{+23.7}$ & $4.0_{-0.0}^{+17.1}$ & $5.7_{-0.0}^{+34.2}$ & $0.1_{-0.0}^{+24.9}$ & $10.0_{-5.5}^{+21.9}$ & $0.3_{-0.0}^{+10.7}$ & $1.1_{-0.0}^{+15.6}$ & $73.7_{-6.5}^{+19.9}$ & $\ldots$ & $\ldots$ & $0.0_{-0.0}^{+20.7}$ \\
\hline 113 & K7 & $0.0_{-0.0}^{+1.2}$ & $0.0_{-0.0}^{+0.7}$ & $3.3_{-0.5}^{+1.7}$ & $4.4_{-1.9}^{+1.3}$ & $24.9_{-10.3}^{+12.9}$ & $10.3_{-3.4}^{+4.9}$ & $5.8_{-2.2}^{+5.2}$ & $0.0_{-0.0}^{+2.0}$ & $42.5_{-17.9}^{+13.4}$ & 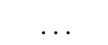 & $\ldots$ & $8.8_{-4.4}^{+7.0}$ \\
\hline$\ldots$ & & $36.9_{-14.1}^{+19.9}$ & $2.1_{-0.0}^{+8.1}$ & $3.6_{-1.4}^{+4.2}$ & $0.0_{-0.0}^{+2.6}$ & $36.2_{-13.5}^{+21.8}$ & $1.7_{-0.0}^{+9.0}$ & $0.0_{-0.0}^{+5.8}$ & $0.0_{-0.0}^{+7.9}$ & $10.9_{-1.3}^{+11.9}$ & $\ldots$ & $\ldots$ & $8.6_{-4.2}^{+10.8}$ \\
\hline 114 & F9 & $0.4_{-0.0}^{+5.3}$ & $0.0_{-0.0}^{+0.5}$ & $2.8_{-1.1}^{+1.2}$ & $0.1_{-0.0}^{+0.8}$ & $34.7_{-12.5}^{+17.7}$ & $4.2_{-2.8}^{+3.2}$ & $1.9_{-0.6}^{+6.6}$ & $0.0_{-0.0}^{+2.3}$ & $51.8_{-17.1}^{+15.2}$ & $\ldots$ & $\ldots$ & $17.4_{-5.2}^{+10.1}$ \\
\hline 115 & M0.5 & $3.5_{-2.2}^{+6.8}$ & $0.4_{-0.2}^{+1.2}$ & $1.5_{-0.0}^{+1.5}$ & $0.6_{-0.0}^{+1.2}$ & $65.7_{-48.2}^{+17.3}$ & $0.0_{-0.0}^{+4.3}$ & $0.0_{-0.0}^{+4.3}$ & $0.0_{-0.0}^{+3.5}$ & $25.6_{-14.5}^{+10.3}$ & $\ldots$ & $\ldots$ & $2.6_{-0.9}^{+4.6}$ \\
\hline 117 & $\mathrm{~K} 2$ & $74.6_{-31.9}^{+32.8}$ & $1.4_{-0.0}^{+3.6}$ & $5.9_{-4.1}^{+2.1}$ & $0.0_{-0.0}^{+1.6}$ & $5.1_{-0.2}^{+6.4}$ & $10.7_{-3.2}^{+2.1}$ & $0.0_{-0.0}^{+0.9}$ & $0.0_{-0.0}^{+0.9}$ & $\begin{array}{l}2.1_{-0.0}^{+2.0} \\
\text { 2. }\end{array}$ & $\ldots$ & $\ldots$ & $0.2_{-0.0}^{+1.2}$ \\
\hline$\ldots$ & & $37.9_{-15.7}^{+14.9}$ & $6.3_{-4.7}^{+5.5}$ & $19.0_{-9.9}^{+9.1}$ & $6.1_{-4.1}^{+5.3}$ & $23.6_{-6.8}^{+14.9}$ & $0.8_{-0.4}^{+2.1}$ & $0.0_{-0.0}^{+6.8}$ & $\begin{array}{l}2.3_{-2.4}^{+3.9} \\
\end{array}$ & $2.4_{-1.3}^{+2.8}$ & $\ldots$ & $\ldots$ & $1.7_{-0.9}^{+2.2}$ \\
\hline 119 & K7 & $0.0_{-0.0}^{+11.2}$ & $0.7_{-0.0}^{+23.7}$ & $1.1_{-0.0}^{+17.4}$ & $1.9_{-0.0}^{+31.6}$ & $11.1_{-2.7}^{+56.7}$ & $0.2_{-0.0}^{+25.1}$ & $0.6_{-0.0}^{+41.3}$ & $0.3_{-0.0}^{+33.6}$ & $84.0_{-26.7}^{+58.6}$ & $\ldots$ & $\ldots$ & $0.0_{-0.0}^{+96.2}$ \\
\hline 122 & M0 & $30.7_{-30.4}^{+3.5}$ & $0.0_{-0.0}^{+7.8}$ & $1.4_{-0.0}^{+10.5}$ & $0.0_{-0.0}^{+7.1}$ & $52.6_{-35.4}^{+10.1}$ & $6.4_{-3.1}^{+15.0}$ & $0.0_{-0.0}^{+10.0}$ & $0.0_{-0.0}^{+4.4}$ & $1.7_{-1.0}^{+17.3}$ & $\ldots$ & $\ldots$ & $7.1_{-1.1}^{+14.6}$ \\
\hline$\ldots$ & & $25.3_{-12.9}^{+38.5}$ & $0.2_{-0.6}^{+7.5}$ & $5.5_{-0.0}^{+19.7}$ & $22.7_{-14.1}^{+36.9}$ & $27.8_{-13.3}^{+39.9}$ & $0.9_{-0.0}^{+11.2}$ & $0.0_{-0.0}^{+14.3}$ & $0.0_{-1.7}^{+10.5}$ & $11.8_{-4.3}^{+13.6}$ & $\ldots$ & $\ldots$ & $5.7_{-0.0}^{+18.0}$ \\
\hline 123 & M0 & $42.0_{-19.7}^{+65.0}$ & $0.7_{-0.0}^{+3.3}$ & $5.4_{-3.7}^{+2.7}$ & $0.0_{-0.0}^{+1.4}$ & $41.2_{-16.8}^{+11.7}$ & $4.1_{-0.6}^{+6.4}$ & $0.0_{-0.0}^{+4.2}$ & $2.7_{-1.4}^{+3.7}$ & $4.0_{-0.1}^{+6.8}$ & $\ldots$ & $\ldots$ & $0.0_{-0.0}^{+0.5}$ \\
\hline 125 & M0 & $4.9_{-0.0}^{+13.0}$ & $0.0_{-0.0}^{+3.3}$ & $2.3_{-1.6}^{+2.5}$ & $0.0_{-0.0}^{+1.9}$ & $56.9_{-23.8}^{+11.9}$ & $0.0_{-0.0}^{+5.0}$ & $0.0_{-0.0}^{+7.2}$ & $0.0_{-0.0}^{+4.4}$ & $30.8_{-16.9}^{+6.4}$ & $\ldots$ & $\ldots$ & $5.0_{-1.2}^{+11.2}$ \\
\hline$\cdots$ & & $31.0_{-20.4}^{+10.3}$ & $11.6_{-12.8}^{+1.5}$ & $0.0_{-2.6}^{+0.9}$ & $0.0_{-0.0}^{+2.5}$ & $29.1_{-13.7}^{+9.2}$ & $7.0_{-5.2}^{+4.1}$ & $0.2_{-0.0}^{+1.8}$ & $0.0_{-0.0}^{+3.8}$ & $16.0_{-7.7}^{+6.1}$ & $\ldots$ & $\ldots$ & $5.1_{-3.1}^{+3.2}$ \\
\hline 127 & M2 & $1.5_{-0.0}^{+2.9}$ & $3.4_{-2.6}^{+1.2}$ & $0.0_{-0.0}^{+2.0}$ & $0.9_{-0.4}^{+1.9}$ & $0.0_{-0.0}^{+2.0}$ & $0.0_{-0.0}^{+3.1}$ & $1.5_{-0.0}^{+5.7}$ & $1.3_{-0.5}^{+4.4}$ & $91.5_{-21.7}^{+71.4}$ & $\ldots$ & $\ldots$ & $0.0_{-0.0}^{+7.1}$ \\
\hline 129 & $\ldots$ & $0.2_{-0.0}^{+2.4}$ & $0.0_{-0.0}^{+0.8}$ & $0.9_{-0.0}^{+1.7}$ & $0.0_{-0.0}^{+1.3}$ & $14.7_{-4.2}^{+7.3}$ & $1.7_{-0.0}^{+4.2}$ & $0.0_{-0.0}^{+2.3}$ & $0.0_{-0.0}^{+0.9}$ & $82.5_{-55.0}^{+20.0}$ & $\ldots$ & $\ldots$ & $0.0_{-0.0}^{+6.7}$ \\
\hline 137 & $\ldots$ & $40.7_{-12.0}^{+10.3}$ & $0.8_{-0.0}^{+6.0}$ & $0.9_{-0.0}^{+2.9}$ & $0.0_{-0.0}^{+2.3}$ & $39.0_{-19.2}^{+10.8}$ & $5.6_{-4.2}^{+3.4}$ & $5.8_{-3.6}^{+4.1}$ & $0.0_{-0.0}^{+3.2}$ & $0.0_{-0.0}^{+2.3}$ & $\ldots$ & $\ldots$ & $7.1_{-2.1}^{+4.7}$ \\
\hline 142 & M4 & $\begin{array}{l}0.7_{-0.0}^{+6.3} \\
0\end{array}$ & $0.5_{-0.0}^{-2.0}$ & $2.6_{-1.1}^{+2.3}$ & $\begin{array}{l}-0.0 \\
1.3_{-0.2}^{+2.9}\end{array}$ & $\begin{array}{r}58.7_{-33.8}^{+20.2} \\
\end{array}$ & $4.0_{-2.6}^{+6.6}$ & $1.9_{-0.0}^{+7.4}$ & $4.5_{-2.7}^{+4.9}$ & $25.7_{-10.1}^{+9.0}$ & $\ldots$ & $\ldots$ & $0.1_{-0.0}^{+6.1}$ \\
\hline 144 & $\ldots$ & $0.0_{-0.0}^{+5.3}$ & $0.0_{-0.0}^{+2.1}$ & $6.1_{-1.3}^{+1.6}$ & $4.4_{-1.9}^{+2.6}$ & $46.2_{-23.0}^{+6.0}$ & $11.2_{-3.3}^{+4.4}$ & $0.0_{-0.0}^{+3.0}$ & $0.0_{-0.0}^{+3.0}$ & $32.0_{-7.4}^{+13.9}$ & $\ldots$ & $\ldots$ & $0.0_{-0.0}^{+2.6}$ \\
\hline .. & & $23.3_{-9.5}^{+91.2}$ & $22.1_{-11.6}^{+33.3}$ & $10.9_{-9.9}^{+26.9}$ & $0.0_{-0.0}^{+41.9}$ & $7.3_{-7.8}^{+21.8}$ & $10.5_{-6.9}^{+20.1}$ & $12.1_{-6.2}^{+10.9}$ & $0.0_{-0.0}^{+22.2}$ & $8.3_{-3.5}^{+19.9}$ & $\ldots$ & $\ldots$ & $5.5_{-3.7}^{+14.3}$ \\
\hline 146 & M4 & $6.8_{-1.1}^{+2.7}$ & $0.0_{-0.0}^{+0.6}$ & $4.4_{-1.6}^{+1.2}$ & $2.2_{-1.1}^{+1.1}$ & $34.1_{-6.7}^{+4.5}$ & $0.0_{-0.0}^{+1.1}$ & $4.1_{-2.3}^{+3.0}$ & $0.0_{-0.0}^{+1.3}$ & $32.6_{-7.7}^{+8.3}$ & $\ldots$ & $\ldots$ & $15.6_{-4.7}^{+2.9}$ \\
\hline$\ldots$ & & $17.2_{-2.6}^{+6.3}$ & $0.0_{-0.0}^{+4.2}$ & $0.0_{-0.0}^{+3.8}$ & $0.0_{-0.0}^{+4.7}$ & $27.7_{-8.0}^{+8.1}$ & $0.0_{-0.0}^{+3.8}$ & $2.2_{-0.0}^{+1.5}$ & $0.0_{-0.0}^{+2.6}$ & $52.9_{-10.5}^{+19.8}$ & $\ldots$ & $\ldots$ & $0.0_{-0.0}^{+8.7}$ \\
\hline 147 & $\ldots$ & $0.0_{-0.0}^{+4.5}$ & $0.0_{-0.0}^{+2.9}$ & $3.3_{-1.3}^{+2.8}$ & $1.3_{-0.9}^{+1.6}$ & $34.1_{-10.9}^{+18.9}$ & $9.8_{-4.6}^{+9.0}$ & $0.0_{-0.6}^{+3.5}$ & $0.7_{-0.0}^{+4.8}$ & $50.7_{-41.6}^{+18.1}$ & $\ldots$ & $\ldots$ & $0.0_{-0.0}^{+15.4}$ \\
\hline 148 & K7 & $7.4_{-0.0}^{+15.4}$ & $2.5_{-1.3}^{+2.7}$ & $3.5_{-0.8}^{+3.4}$ & $0.0_{-0.0}^{+1.0}$ & $70.6_{-49.9}^{+18.2}$ & $3.7_{-2.0}^{+8.0}$ & $0.0_{-0.0}^{+5.3}$ & $2.5_{-0.0}^{+5.1}$ & $5.1_{-0.0}^{+8.9}$ & $\ldots$ & $\ldots$ & $4.6_{-1.1}^{+5.1}$ \\
\hline$\cdots$ & & $3.8_{-0.0}^{+36.6}$ & $17.8_{-8.3}^{+16.9}$ & $30.8_{-11.4}^{+24.5}$ & $1.9_{-0.0}^{+10.7}$ & $2.0_{-0.0}^{+13.4}$ & $10.9_{-7.5}^{+16.2}$ & $5.0_{-3.7}^{+17.7}$ & $2.9_{-0.0}^{+21.5}$ & $24.7_{-9.6}^{+18.4}$ & $\ldots$ & $\ldots$ & $0.1_{-0.0}^{+4.7}$ \\
\hline 149 & M0 & $37.8_{-13.3}^{+22.2}$ & $0.7_{-0.0}^{+2.9}$ & $1.4_{-0.9}^{+2.8}$ & $0.0_{-0.0}^{+1.6}$ & $24.1_{-10.7}^{+10.8}$ & $3.9_{-1.6}^{+5.5}$ & $0.0_{-0.0}^{+2.3}$ & $1.3_{-0.0}^{+2.6}$ & $30.1_{-8.9}^{+11.2}$ & $\ldots$ & $\ldots$ & $0.4_{-0.0}^{+8.8}$ \\
\hline$\cdots$ & & $41.7_{-28.1}^{+24.7}$ & $0.0_{-0.0}^{+5.5}$ & $4.1_{-1.5}^{+10.3}$ & $4.4_{-3.6}^{+8.6}$ & $28.3_{-21.1}^{+14.5}$ & $3.7_{-0.5}^{+13.0}$ & $1.1_{-0.1}^{+12.7}$ & $0.0_{-0.0}^{+8.3}$ & $\begin{array}{l}-0.9 \\
8.8_{-1.2}^{+6.5}\end{array}$ & $\ldots$ & $\ldots$ & $7.8_{-1.9}^{+8.4}$ \\
\hline \multicolumn{14}{|c|}{ Taurus } \\
\hline $04108+2910$ & M0 & $0.1_{-0.0}^{+0.4}$ & $0.5_{-0.0}^{+1.4}$ & $2.9_{-1.0}^{+1.2}$ & $0.0_{-0.0}^{+0.8}$ & $10.4_{-6.1}^{+4.1}$ & $11.2_{-4.7}^{+3.1}$ & $3.6_{-1.6}^{+3.1}$ & $0.0_{-0.0}^{+0.9}$ & $71.3_{-29.0}^{+16.2}$ & . & $\cdots$ & $0.0_{-0.0}^{+8.4}$ \\
\hline$\ldots$ & & $13.2_{-5.6}^{+9.3}$ & $0.1_{-0.0}^{+6.5}$ & $4.0_{-0.0}^{+10.8}$ & $0.0_{-0.0}^{+9.9}$ & $58.5_{-17.8}^{+22.3}$ & $0.0_{-0.0}^{+8.0}$ & $0.0_{-0.0}^{+7.8}$ & $0.0_{-0.0}^{+45.4}$ & $12.9_{-5.3}^{+15.9}$ & $\ldots$ & $\ldots$ & $11.4_{-6.4}^{+7.1}$ \\
\hline $04200+2759$ & $\ldots$ & $4.7_{-2.4}^{+1.5}$ & $0.1_{-0.0}^{+2.2}$ & $0.4_{-0.0}^{+1.2}$ & $0.0_{-0.0}^{+0.9}$ & $48.4_{-13.1}^{+14.7}$ & $7.9_{-4.1}^{+6.2}$ & $2.8_{-0.0}^{+5.3}$ & $0.0_{-0.0}^{+2.5}$ & $19.6_{-5.6}^{9.4}$ & $\ldots$ & $\ldots$ & $16.0_{-11.1}^{+7.1}$ \\
\hline$\cdots$ & & $14.3_{-7.3}^{+11.5}$ & $1.2_{-7.9}^{+4.5}$ & $4.2_{-3.6}^{+5.1}$ & $3.8_{-7.2}^{+8.2}$ & $58.6_{-26.4}^{+32.2}$ & $0.0_{-0.0}^{+9.8}$ & $0.0_{-0.0}^{+18.6}$ & $7.2_{-20.3}^{+31.8}$ & $10.3_{-5.6}^{+7.3}$ & $\ldots$ & $\ldots$ & $0.4_{-1.4}^{+2.6}$ \\
\hline $04216+2603$ & M1 & $43.9_{-22.8}^{+15.3}$ & $1.8_{-0.7}^{+5.4}$ & $2.1_{-1.0}^{+3.0}$ & $0.0_{-0.0}^{+2.2}$ & $18.9_{-12.3}^{+17.6}$ & $6.4_{-5.1}^{+1.7}$ & $0.0_{-0.2}^{+2.5}$ & $0.0_{-0.0}^{+2.8}$ & $27.1_{-9.5}^{+9.7}$ & $\ldots$ & $\ldots$ & $0.0_{-0.0}^{+9.3}$ \\
\hline$\ldots$ & & $18.6_{-9.0}^{+27.5}$ & $0.1_{-0.0}^{+16.8}$ & $13.2_{-10.7}^{+31.5}$ & $1.8_{-8.2}^{+40.3}$ & $4.4_{-5.1}^{+16.5}$ & $2.1_{-3.1}^{+18.1}$ & $1.5_{-2.9}^{+20.5}$ & $10.5_{-6.0}^{+17.2}$ & $44.5_{-17.3}^{+31.4}$ & $\ldots$ & $\ldots$ & $3.2_{-4.3}^{+13.5}$ \\
\hline
\end{tabular}


Table 5

(Continued)

\begin{tabular}{|c|c|c|c|c|c|c|c|c|c|c|c|c|c|}
\hline \multirow[t]{2}{*}{ ID } & \multirow[t]{2}{*}{ SpT } & \multicolumn{4}{|c|}{$0.1(\mu \mathrm{m})$} & \multicolumn{4}{|c|}{$1.5(\mu \mathrm{m})$} & \multicolumn{4}{|c|}{$6.0(\mu \mathrm{m})$} \\
\hline & & $\mathrm{Oli} / \mathrm{Pyr}^{\mathrm{b}}(\%)$ & Ens $(\%)$ & For $(\%)$ & Sil (\%) & Oli/Pyr (\%) & Ens (\%) & For $(\%)$ & Sil (\%) & Oli/Pyr (\%) & Ens $(\%)$ & For $(\%)$ & Sil (\%) \\
\hline $04303+2240$ & $\ldots$ & $2.4_{-0.0}^{+9.1}$ & $0.0_{-0.0}^{+1.7}$ & $2.7_{-0.0}^{+3.6}$ & $0.0_{-0.0}^{+0.7}$ & $74.2_{-17.9}^{+25.2}$ & $3.5_{-0.0}^{+8.5}$ & $9.6_{-6.3}^{+10.7}$ & $2.0_{-0.4}^{+4.0}$ & $0.0_{-0.0}^{+1.0}$ & $\cdots$ & $\cdots$ & $5.5_{-0.0}^{+9.8}$ \\
\hline $04370+2559$ & $\cdots$ & $3.1_{-0.0}^{+7.3}$ & $1.2_{-0.0}^{+2.3}$ & $0.7_{-0.0}^{+1.5}$ & $0.0_{-0.0}^{+0.4}$ & $80.2_{-33.6}^{+16.5}$ & $0.3_{-0.0}^{+7.0}$ & $1.2_{-0.0}^{+5.7}$ & $0.0_{-0.0}^{+2.1}$ & $4.9_{-0.0}^{+5.3}$ & $\cdots$ & $\because$ & $8.3_{-2.3}^{+2.8}$ \\
\hline $04385+2550$ & M0 & $16.2_{-7.9}^{+2.3}$ & $0.8_{-0.5}^{+1.3}$ & $1.0_{-0.0}^{+1.9}$ & $0.0_{-0.0}^{+0.7}$ & $55.1_{-12.6}^{+12.3}$ & $4.8_{-3.0}^{+4.9}$ & $3.7_{-2.4}^{+3.8}$ & $0.0_{-0.0}^{+2.5}$ & $6.4_{-1.7}^{+1.9}$ & $\ldots$ & $\ldots$ & $11.9_{-6.2}^{+2.7}$ \\
\hline$\cdots$ & & $13.2_{-00}^{+11.7}$ & $0.0_{-00}^{+2.2}$ & $1.6_{-00}^{+3.3}$ & $5.7_{-00}^{+11.4}$ & $47.7_{-124}^{+34.5}$ & $0.0_{-00}^{+16.4}$ & $0.0_{-00}^{+9.1}$ & $19.9_{-00}^{+39.6}$ & $8.1_{-16}^{+5.0}$ & $\ldots$ & $\ldots$ & $3.9_{-0.0}^{+6.0}$ \\
\hline AATau & K7 & $12.5_{-0.6}^{+10.1}$ & $0.4_{-00}^{+1.0}$ & $1.4_{-07}^{+1.4}$ & $0.0_{-00}^{+0.7}$ & $30.6_{-84}^{+7.5}$ & $3.4_{-26}^{+2.6}$ & $2.6_{-16}^{+3.3}$ & $2.1_{-08}^{+2.6}$ & $45.7_{-206}^{+13.6}$ & $\ldots$ & $\ldots$ & $1.3_{-06}^{+5.3}$ \\
\hline$\ldots$ & & $2.6_{-00}^{+19.7}$ & $19.2_{-00}^{+15.3}$ & $0.0_{-0.0}^{+24.2}$ & $18.3_{-00}^{+22.4}$ & $9.3_{-0.9}^{+20.9}$ & $8.3_{-0.8}^{+16.8}$ & $4.5_{-0.0}^{+6.0}$ & $7.0_{-00}^{+13.7}$ & $29.6_{-0.0}^{+37.2}$ & $\ldots$ & $\ldots$ & $1.2_{-0.0}^{+24.4}$ \\
\hline BPTau & K7 & $29.9_{-7.9}^{+5.4}$ & $1.1_{-0.6}^{+0.9}$ & $1.0_{-0.0}^{+1.3}$ & $0.0_{-0.0}^{+0.3}$ & $25.4_{-8.4}^{+5.6}$ & $0.8_{-0.0}^{+1.1}$ & $0.0_{-0.0}^{+0.8}$ & $0.0_{-0.0}^{+0.5}$ & $31.6_{-10.3}^{+6.8}$ & $\ldots$ & $\ldots$ & $10.3_{-4.1}^{+2.9}$ \\
\hline CITau & K7 & $2.3_{-0.0}^{+5.6}$ & $0.0_{-0.0}^{+0.3}$ & $2.1_{-0.0}^{+1.0}$ & $0.0_{-0.0}^{+0.9}$ & $51.8_{-12.3}^{+10.5}$ & $4.6_{-1.0}^{+6.6}$ & $2.8_{-1.3}^{+3.7}$ & $0.1_{-0.0}^{+1.5}$ & $17.8_{-7.8}^{+13.8}$ & $\ldots$ & $\ldots$ & $18.7_{-3.9}^{+5.1}$ \\
\hline$\ldots$ & & $14.8_{-7.1}^{+12.4}$ & $0.3_{-0.0}^{+1.5}$ & $3.5_{-0.0}^{+10.3}$ & $7.5_{-0.0}^{+9.9}$ & $14.5_{-4.3}^{+12.3}$ & $1.9_{-0.0}^{+19.1}$ & $0.0_{-0.0}^{+3.4}$ & $9.7_{-5.8}^{+7.2}$ & $27.4_{-11.2}^{+11.9}$ & $\ldots$ & $\ldots$ & $20.3_{-6.0}^{+36.9}$ \\
\hline CWTau & K3 & $8.2_{-2.3}^{+1.9}$ & $1.0_{-0.0}^{+0.7}$ & $1.0_{-0.0}^{+1.5}$ & $0.2_{-0.0}^{+2.4}$ & $23.5_{-5.3}^{+10.5}$ & $1.2_{-0.0}^{+3.5}$ & $0.0_{-0.0}^{+1.1}$ & $0.0_{-0.0}^{+0.8}$ & $46.4_{-19.3}^{+9.9}$ & $\ldots$ & $\ldots$ & $18.5_{-4.7}^{+7.4}$ \\
\hline CoKuTau3 & M1 & $3.3_{-0.5}^{+6.8}$ & $\begin{array}{l}-0.0 \\
0.0_{-0.0}^{+1.0}\end{array}$ & $\begin{array}{r}-0.0 \\
2.7_{-1.4}^{+1.6}\end{array}$ & $0.0_{-0.0}^{+0.3}$ & $63.5_{-24.8}^{-15.3}$ & $\begin{array}{r}-0.0 \\
3.7_{-2.0}^{+4.5}\end{array}$ & $0.4_{-0.0}^{+3.2}$ & $0.0_{-0.0}^{+2.0}$ & $\begin{array}{l}26.0_{-4.4}^{+8.3} \\
2.3\end{array}$ & $\ldots$ & $\ldots$ & $0.3_{-0.0}^{+4.1}$ \\
\hline$\ldots$ & & $15.2_{-6.0}^{+9.6}$ & $5.3_{-0.0}^{+10.2}$ & $3.8_{-2.7}^{+1.9}$ & $0.2_{-0.0}^{+4.6}$ & $24.1_{-8.6}^{+10.5}$ & $1.8_{-0.0}^{+3.0}$ & $14.3_{-4.3}^{+25.3}$ & $4.4_{-2.8}^{+7.3}$ & $22.5_{-10.1}^{+15.4}$ & $\ldots$ & $\cdots$ & $9.0_{-4.9}^{+4.9}$ \\
\hline CoKuTau4 & M1.5 & $24.5_{-17.1}^{+3.5}$ & $0.0_{-0.0}^{+2.7}$ & $1.0_{-0.0}^{+2.2}$ & $0.0_{-0.0}^{+1.0}$ & $44.8_{-12.1}^{+10.8}$ & $3.7_{-1.4}^{+3.5}$ & $3.9_{-2.8}^{+3.9}$ & $2.6_{-2.3}^{+4.5}$ & $6.0_{-0.6}^{+6.9}$ & $\ldots$ & $\ldots$ & $3.6_{-7.1}^{+4.2}$ \\
\hline$\ldots$ & & $13.4_{-5.4}^{+8.4}$ & $0.3_{-0.6}^{+1.3}$ & $0.0_{-0.7}^{+1.4}$ & $13.5_{-6.3}^{+9.1}$ & $5.8_{-2.6}^{+4.0}$ & $0.0_{-0.4}^{+0.7}$ & $0.1_{-0.2}^{+0.7}$ & $9.5_{-4.3}^{+6.4}$ & $53.9_{-17.1}^{+26.6}$ & $\cdots$ & $\cdots$ & $3.5_{-3.2}^{+5.3}$ \\
\hline DDTau & M3 & $10.6_{-2.7}^{+4.7}$ & $2.6_{-0.0}^{+3.8}$ & $2.5_{-0.0}^{+2.6}$ & $0.0_{-0.0}^{+1.4}$ & $50.2_{-24.5}^{+19.1}$ & $5.2_{-3.0}^{+5.6}$ & $2.1_{-1.6}^{+5.1}$ & $1.1_{-0.6}^{+4.2}$ & $1.7_{-0.3}^{+11.5}$ & $\cdots$ & $\cdots$ & $22.9_{-9.0}^{+9.0}$ \\
\hline & & $38.7_{-23.6}^{+26.0}$ & $9.0_{-6.1}^{+8.8}$ & $18.5_{-8.6}^{+11.9}$ & $0.0_{-0.0}^{+5.5}$ & $16.1_{-7.1}^{+9.4}$ & $0.1_{-0.2}^{+1.1}$ & $0.0_{-1.2}^{+2.2}$ & $0.2_{-0.9}^{+3.9}$ & $14.5_{-5.6}^{+4.7}$ & & & $0.0_{-0.0}^{+1.1}$ \\
\hline DETau & M1 & $11.0_{-2.4}^{+6.9}$ & $0.4_{-0.0}^{+1.7}$ & $3.3_{-0.0}^{+2.5}$ & $0.0_{-0.0}^{+0.6}$ & $37.3_{-19.5}^{+6.9}$ & $7.2_{-2.2}^{+3.8}$ & $1.1_{-1.1}^{+3.2}$ & $0.0_{-0.0}^{+1.4}$ & $1.3_{-6.1}^{+6.3}$ & $\cdots$ & $\cdots$ & $8.5_{-3.3}^{+5.4}$ \\
\hline$\ldots$ & & $51.2_{-24.7}^{+22.1}$ & $0.3_{-0.0}^{+6.2}$ & $4.4_{-0.0}^{+6.9}$ & $0.7_{-0.0}^{+10.2}$ & $15.1_{-4.2}^{+11.0}$ & $0.0_{-0.0}^{+5.3}$ & $0.0_{-0.0}^{+2.5}$ & $9.3_{-0.0}^{+15.2}$ & $18.9_{-5.2}^{+14.7}$ & $\cdots$ & $\cdots$ & $0.0_{-0.0}^{+4.6}$ \\
\hline DFTau & M0.5 & $0.6_{-00}^{+8.0}$ & $0.2_{-00}^{+2.3}$ & $1.8_{-00}^{+2.1}$ & $0.3_{-00}^{+3.1}$ & $19.2_{-7.8}^{+3.8}$ & $3.7_{-00}^{+3.9}$ & $0.0_{-00}^{+4.9}$ & $2.8_{-00}^{+6.5}$ & $1.3_{-174}^{+18.8}$ & $\cdots$ & & $0.0_{-00}^{+7.3}$ \\
\hline DHTau & M2 & $2.3_{-0.3}^{+2.1}$ & $1.8_{-0.8}^{+1.8}$ & $5.4_{-1.2}^{+2.4}$ & $1.5_{-0.9}^{+1.6}$ & $29.6_{-10.3}^{+9.8}$ & $11.9_{-3.1}^{+5.6}$ & $0.0_{-0.4}^{+2.2}$ & $1.1_{-1.0}^{+2.2}$ & $44.6_{-22.4}^{+17.1}$ & $\ldots$ & $\ldots$ & $0.0_{-0.0}^{+9.9}$ \\
\hline$\ldots$ & & $32.0_{-12.3}^{+16.2}$ & $2.5_{-1.7}^{+6.0}$ & $3.4_{-3.1}^{+8.5}$ & $0.0_{-1.3}^{+5.6}$ & $17.3_{-11.8}^{+20.6}$ & $5.7_{-5.9}^{+17.2}$ & $4.3_{-3.2}^{+8.0}$ & $0.0_{-7.3}^{+2.9}$ & $22.4_{-8.0}^{++9.4}$ & $\ldots$ & $\ldots$ & $12.4_{-7.4}^{+10.2}$ \\
\hline DKTau & M0 & $13.0_{-3.1}^{+3.8}$ & $0.6_{-0.0}^{+0.9}$ & $1.9_{-1.2}^{+1.3}$ & $3.3_{-0.9}^{+1.3}$ & $54.6_{-12.9}^{+10.1}$ & $3.1_{-1.9}^{+2.7}$ & $0.0_{-0.0}^{+1.2}$ & $1.8_{-0.0}^{+1.8}$ & $17.2_{-3.7}^{+3.1}$ & $\cdots$ & $\cdots$ & $4.2_{-1.1}^{+1.4}$ \\
\hline DLTau & K7 & $0.1_{-1.5}^{+5.6}$ & $0.0_{-0.0}^{+1.1}$ & $3.0_{-1.2}^{+2.3}$ & $5.0_{-2.2}^{+3.5}$ & $38.0_{-25.0}^{+17.1}$ & $10.1_{-5.6}^{+10.2}$ & $0.0_{-0.0}^{+6.0}$ & $8.8_{-4.7}^{+7.6}$ & $24.4_{-8.5}^{+12.3}$ & $\cdots$ & & $10.7_{-4.7}^{+7.4}$ \\
\hline DMTau & M1 & $4.1_{-0.0}^{+13.6}$ & $0.0_{-0.0}^{+1.8}$ & $4.7_{-1.0}^{+2.3}$ & $0.0_{-0.0}^{+1.2}$ & $18.0_{-13.8}^{+8.5}$ & $16.6_{-7.4}^{+6.6}$ & $2.8_{-1.4}^{+5.4}$ & $0.0_{-0.0}^{+2.1}$ & $36.8_{-10.8}^{+14.4}$ & $\ldots$ & $\ldots$ & $17.0_{-12.1}^{+8.3}$ \\
\hline$\cdots$ & & $42.6_{-17.1}^{+28.1}$ & $0.7_{-0.0}^{+8.9}$ & $1.2_{-0.0}^{+3.6}$ & $5.3_{-4.8}^{+12.2}$ & $2.0_{-1.0}^{+2.6}$ & $0.8_{-0.0}^{+2.8}$ & $2.1_{-0.0}^{+5.9}$ & $8.8_{-4.7}^{+9.7}$ & $29.7_{-10.3}^{+21.5}$ & $\cdots$ & $\cdots$ & $6.8_{-5.0}^{+4.2}$ \\
\hline DNTau & M0 & $3.4_{-0.0}^{+5.1}$ & $0.0_{-0.0}^{+0.6}$ & $2.2_{-0.6}^{+1.4}$ & $1.4_{-0.9}^{+1.1}$ & $27.6_{-3.5}^{+11.4}$ & $3.1_{-2.0}^{+2.7}$ & $1.4_{-0.9}^{+1.9}$ & $0.0_{-0.0}^{+2.1}$ & $43.8_{-26.5}^{+23.9}$ & & & $17.0_{-4.4}^{+13.5}$ \\
\hline$\ldots$ & & $33.7_{-0.0}^{+39.1}$ & $0.2_{-0.0}^{+9.1}$ & $0.0_{-0.0}^{+18.1}$ & $0.0_{-0.0}^{+7.6}$ & $46.4_{-0.0}^{+53.9}$ & $0.0_{-0.0}^{+38.7}$ & $3.6_{-0.0}^{+16.8}$ & $0.0_{-0.0}^{+>1.0}$ & $3.2_{-0.0}^{+11.2}$ & $\cdots$ & $\cdots$ & $12.9_{-0.0}^{+32.7}$ \\
\hline DOTau & M0 & $1.2_{-0.0}^{+8.4}$ & $0.0_{-0.0}^{+3.8}$ & $1.6_{-0.0}^{+2.7}$ & $0.3_{-0.0}^{+4.0}$ & $52.2_{-9.1}^{+28.2}$ & $4.5_{-0.0}^{+5.7}$ & $5.3_{-0.0}^{+6.3}$ & $1.9_{-0.0}^{+6.2}$ & $19.9_{-7.5}^{+14.9}$ & $\cdots$ & $\ldots$ & $13.1_{-4.6}^{+5.0}$ \\
\hline DPTau & M0.5 & $35.1_{-15.8}^{+13.7}$ & $0.0_{-0.0}^{+1.5}$ & $1.1_{-0.0}^{+1.9}$ & $0.0_{-0.0}^{+1.6}$ & $50.7_{-15.8}^{+27.5}$ & $0.3_{-0.0}^{+2.8}$ & $1.5_{-0.0}^{+4.0}$ & $0.0_{-0.0}^{+1.8}$ & $0.2_{-0.0}^{+1.6}$ & & & $11.0_{-0.0}^{+5.6}$ \\
\hline$\ldots$ & & $20.1_{-8.0}^{+8.9}$ & $9.8_{-5.1}^{+4.6}$ & $3.0_{-2.1}^{+3.7}$ & $7.7_{-3.8}^{+3.8}$ & $44.4_{-23.3}^{+18.0}$ & $0.0_{-1.6}^{+5.4}$ & $7.8_{-3.8}^{+5.1}$ & $0.0_{-0.3}^{+2.7}$ & $4.0_{-1.0}^{+1.2}$ & $\cdots$ & . . & $3.1_{-0.0}^{+2.3}$ \\
\hline DQTau & M0 & $\begin{array}{l}1.2 \\
1.3 \\
.1 .3\end{array}$ & $5.5_{-1.4}^{+1.7}$ & $2.0_{-0.7}^{+1.1}$ & $0.0_{-0.0}^{+0.6}$ & 8.1 & $6.0_{-2.0}^{+3.1}$ & $0.0_{-0.0}^{+1.4}$ & $1.7_{-1.0}^{+2.8}$ & $62.3_{-34.4}^{+22.9}$ & $\ldots$ & $\cdots$ & $0.0_{-0.0}^{+5.8}$ \\
\hline$\ldots$ & & $14.4_{-5.6}^{+12.0}$ & $0.1_{-0.0}^{+8.7}$ & $0.0_{-0.0}^{+8.0}$ & $0.0_{-0.0}^{+3.3}$ & $30.8_{-14.5}^{-36.9}$ & $0.0_{-0.0}^{+12.6}$ & $4.6_{-3.1}^{+8.4}$ & $0.0_{-0.2}^{+1.11 .7}$ & $21.1_{-10.5}^{+36.4}$ & $\ldots$ & $\ldots$ & $29.1_{-11.9}^{+21.2}$ \\
\hline DRTau & K7 & $25.0_{-5.3}^{+5.8}$ & $0.0_{-0.0}^{+0.7}$ & $1.0_{-0.0}^{+1.5}$ & $0.0_{-0.0}^{+0.6}$ & $31.6_{-8.7}^{+9.3}$ & $0.6_{-0.0}^{+1.5}$ & $1.6_{-1.1}^{+2.1}$ & $0.0_{-0.0}^{+1.0}$ & $20.4_{-9.6}^{+9.9}$ & $\ldots$ & $\ldots$ & $19.8_{-4.7}^{+6.4}$ \\
\hline DSTau & K5 & $35.9_{-6.3}^{+7.1}$ & $0.8_{-0.7}^{+2.9}$ & $2.8_{-1.7}^{+2.3}$ & $0.2_{-0.0}^{+1.2}$ & $17.4_{-4.1}^{+13.7}$ & $6.2_{-3.1}^{+3.4}$ & $0.2_{-0.0}^{+1.4}$ & $0.0_{-0.0}^{+1.1}$ & $36.4_{-10.5}^{+11.9}$ & $\ldots$ & $\ldots$ & $0.0_{-0.0}^{+4.5}$ \\
\hline & & $22.0_{-10.3}^{+14.7}$ & $0.2_{-1.8}^{+3.5}$ & $2.7_{-1.8}^{+3.3}$ & $17.2_{-9.7}^{+14.0}$ & $13.4_{-6.7}^{+10.9}$ & $20.1_{-10.8}^{+17.0}$ & $0.1_{-1.4}^{+3.6}$ & $7.0_{-4.8}^{+7.3}$ & $11.0_{-4.9}^{+7.6}$ & $\ldots$ & & $6.3_{-4.1}^{+6.0}$ \\
\hline F04147+2822 & M4 & $10.2_{-0.0}^{+6.3}$ & $0.0_{-0.0}^{+4.3}$ & $2.9_{-0.0}^{+2.5}$ & $0.0_{-0.0}^{+1.4}$ & $71.2_{-25.9}^{+11.8}$ & $0.0_{-0.0}^{+4.4}$ & $0.0_{-0.0}^{+4.0}$ & $0.1_{-0.0}^{+2.8}$ & $3.6_{-0.1}^{+3.4}$ & $\ldots$ & $\ldots$ & $12.0_{-3.4}^{+5.3}$ \\
\hline F04192+2647 & $\ldots$ & $3.4_{-0.0}^{+12.4}$ & $0.1_{-0.0}^{+0.8}$ & $3.6_{-0.8}^{+1.7}$ & $0.0_{-0.0}^{+0.7}$ & $49.0_{-11.6}^{+10.0}$ & $8.7_{-4.0}^{+3.6}$ & $0.9_{-0.5}^{+3.3}$ & $0.7_{-0.1}^{+4.2}$ & $21.5_{-9.9}^{+11.8}$ & $\ldots$ & $\ldots$ & $12.1_{-4.0}^{+4.2}$ \\
\hline$\ldots$ & & $19.7_{-7.4}^{+16.8}$ & $0.0_{-1.6}^{+9.0}$ & $14.2_{-7.1}^{+15.3}$ & $3.7_{-4.5}^{+9.4}$ & $16.1_{-6.0}^{+12.1}$ & $\begin{array}{l}5.3_{-3.2}^{+9.9} \\
\text { (1) }\end{array}$ & $11.7_{-7.1}^{+16.0}$ & $0.0_{-0.0}^{+5.8}$ & $19.4_{-7.1}^{+12.2}$ & $\ldots$ & $\ldots$ & $9.7_{-4.8}^{+8.5}$ \\
\hline F04262+2654 & $\ldots$ & $0.0_{-0.0}^{+1.0}$ & $2.1_{-0.9}^{+2.0}$ & $0.0_{-0.0}^{+1.2}$ & $3.9_{-2.4}^{+2.8}$ & $53.7_{-16.5}^{+59.3}$ & $14.9_{-5.7}^{+14.3}$ & $0.0_{-0.0}^{+8.6}$ & $3.7_{-3.1}^{+9.6}$ & $19.1_{-8.9}^{+12.1}$ & $\cdots$ & $\cdots$ & $2.6_{-2.2}^{+7.6}$ \\
\hline F04297+2246 & $\ldots$ & $5.7_{-1.3}^{+3.7}$ & $0.0_{-0.0}^{+1.5}$ & $2.6_{-1.5}^{+2.0}$ & $0.3_{-0.0}^{+1.6}$ & $34.0_{-16.4}^{+7.8}$ & $9.5_{-2.9}^{+4.4}$ & $2.8_{-1.9}^{+4.4}$ & $0.0_{-0.0}^{+3.2}$ & $44.1_{-26.1}^{+9.8}$ & $\ldots$ & $\ldots$ & $0.9_{-1.0}^{+12.5}$ \\
\hline .. & & $24.7_{-6.4}^{+61.7}$ & $3.5_{-0.0}^{+15.1}$ & $0.1_{-0.0}^{+32.3}$ & $0.0_{-0.0}^{+17.6}$ & $22.8_{-0.0}^{+66.2}$ & $0.0_{-0.0}^{+26.4}$ & $18.7_{-15.3}^{+18.5}$ & $5.4_{-0.0}^{+35.6}$ & $21.3_{-5.7}^{+29.8}$ & $\cdots$ & $\cdots$ & $3.5_{-0.0}^{+23.3}$ \\
\hline $\mathrm{F} 04297+2246 \mathrm{~A}$ &. & $6.0_{-1.5}^{+4.4}$ & $3.6_{-1.8}^{+1.8}$ & $3.1_{-0.7}^{+1.0}$ & $0.0_{-0.0}^{+0.9}$ & $45 . ._{-10.3}^{+9.6}$ & $9.0_{-3.1}^{+2.9}$ & $3.4_{-2.4}^{+3.0}$ & $2.9_{-1.5}^{+2.3}$ & $23.9_{-5.3}^{+4.1}$ & $\cdots$ & $\cdots$ & $2.1_{-0.0}^{+8.0}$ \\
\hline & & $\begin{array}{l}15.2 \\
10.4\end{array}$ & $1.4_{-0.0}^{+2.8}$ & 2. & $0.0_{-0.0}^{+8.2}$ & & $0.2_{-0.0}^{+2.4}$ & $4.3_{-0.0}^{+5.4}$ & $\begin{array}{l}1.4 \\
0.0\end{array}$ & 5.2 & $\ldots$ & $\ldots$ & $29.2_{-15.7}^{+15.0}$ \\
\hline F04570+2520 & . & $9_{-0}^{+11.6}$ & $0.1_{-0.0}^{+1.7}$ & $0.1_{-0.0}^{+3.9}$ & $0.0_{-0.0}^{+2.9}$ & $\begin{array}{l}+24.3 \\
-22.8 \\
\end{array}$ & $5.8_{-5.2}^{+6.2}$ & $6.6_{-39}^{+6.4}$ & $0.0_{-0.0}^{+4.7}$ & $.5_{-4.3}^{+11.0}$ & $\cdots$ & .. & $0.0_{-0.3}^{+3.2}$ \\
\hline & & $\begin{array}{l}+4.1 \\
-8.8\end{array}$ & $0.0_{-1.9}^{+1.8}$ & $0.0_{-0.0}^{+9.0}$ & $40.0_{-30.3}^{+8.3}$ & $0.0_{-0.9}^{+3.6}$ & $0.0_{-0.0}^{+7.3}$ & $13.7_{-13.9}^{+9.1}$ & $15.7_{-12.3}^{+7.4}$ & 9.96 & & & $9.0_{-7.4}^{+2.6}$ \\
\hline FMTau & M0 & $56.0_{-10.3}^{+14.4}$ & $0.0_{-0.0}^{+2.4}$ & $2.1_{-1.5}^{+2.6}$ & $0.0_{-0.0}^{+1.7}$ & $12.4_{-9.9}^{+2.5}$ & $8.2_{-3.6}^{+3.2}$ & $3.3_{-1.4}^{+6.0}$ & $0.0_{-0.0}^{+1.5}$ & $13.0_{-5.4}^{+5.8}$ & $\cdots$ & $\ldots$ & $5.0_{-0.7}^{+7.4}$ \\
\hline 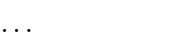 & & $25.0_{-0.0}^{+46.8}$ & $2.4_{-0.0}^{+23.7}$ & $3.5_{-0.0}^{+4.5}$ & $1.2_{-0.0}^{+17.8}$ & $6.4_{-0.0}^{+10.5}$ & $1.1_{-0.0}^{+3.7}$ & $1.3_{-0.0}^{+17.3}$ & $5.4_{-0.0}^{+1.0}$ & $28.3_{-9.4}^{+17.3}$ & $\ldots$ & $\ldots$ & $25.4_{-7.9}^{+45.6}$ \\
\hline FNTau & M5 & $11.3_{-2.0}^{+3.5}$ & $4.5_{-1.4}^{+2.7}$ & $2.7_{-1.0}^{+2.0}$ & $0.1_{-0.2}^{+2.0}$ & $47.1_{-14.4}^{+7.4}$ & $10.9_{-3.7}^{+5.0}$ & $0.1_{-0.0}^{+3.9}$ & $3.2_{-2.3}^{+3.2}$ & $20.1_{-7.2}^{+2.7}$ & $\ldots$ & $\ldots$ & $0.0_{-0.0}^{+14.5}$ \\
\hline 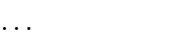 & & $10.6_{-0.0}^{+52.1}$ & $9.3_{-0.0}^{+29.6}$ & $12.6_{-0.0}^{+37.1}$ & $0.0_{-0.0}^{+22.9}$ & $19.0_{-0.0}^{+68.3}$ & $1.3_{-0.0}^{+16.2}$ & $2.9_{-0.0}^{+14.3}$ & $1.8_{-0.0}^{+36.4}$ & $18.8_{-0.0}^{+56.1}$ & $\cdots$ & $\ldots$ & $23.6_{-0.0}^{+102.6}$ \\
\hline FOTau & M2 & $12.4_{-6.2}^{+6.8}$ & $0.0_{-0.0}^{+4.2}$ & $2.5_{-0.0}^{+6.5}$ & $0.2_{-0.0}^{+3.3}$ & $20.6_{-12.0}^{+10.7}$ & $6.6_{-2.3}^{+10.5}$ & $0.0_{-0.0}^{+5.4}$ & $5.6_{-0.0}^{+13.5}$ & $52.1_{-18.6}^{+23.4}$ & $\cdots$ & $\cdots$ & $0.0_{-0.0}^{+19.4}$ \\
\hline FPTau & M4 & $25.8_{-5.8}^{+9.3}$ & $3.7_{-2.4}^{+2.6}$ & $0.9_{-0.4}^{+1.6}$ & $0.0_{-0 .}^{+2.4}$ & $5.5_{-3.6}^{+11.8}$ & $0.0_{-0.2}^{+3.2}$ & $0.0_{-0.0}^{+1.7}$ & $0.0_{-0.0}^{+1.1}$ & $64.1_{-20.7}^{+25.1}$ & & & $0.0_{-1.1}^{+11.6}$ \\
\hline & & $8.6_{-25}^{+8.4}$ & $7.2_{-3.7}^{+6.1}$ & $2.4_{-1.7}^{+2.7}$ & $10.1_{-0.0}^{+6.6}$ & $6.3_{-35}^{+5.5}$ & $15.5_{-10.1}^{+11.9}$ & $2.8_{-0.0}^{+1.8}$ & $8.5_{-0.0}^{+9.4}$ & $33.5_{-9.2}^{+18.2}$ & $\cdots$ & $\cdots$ & $5.0_{-0.0}^{+4.6}$ \\
\hline
\end{tabular}


Table 5

(Continued)

\begin{tabular}{|c|c|c|c|c|c|c|c|c|c|c|c|c|c|}
\hline \multirow[t]{2}{*}{ ID } & \multirow[t]{2}{*}{$\mathrm{SpT}$} & \multicolumn{4}{|c|}{$0.1(\mu \mathrm{m})$} & \multicolumn{4}{|c|}{$1.5(\mu \mathrm{m})$} & \multicolumn{4}{|c|}{$6.0(\mu \mathrm{m})$} \\
\hline & & Oli/Pyr ${ }^{\mathrm{b}}(\%)$ & Ens $(\%)$ & For $(\%)$ & $\operatorname{Sil}(\%)$ & Oli/Pyr (\%) & Ens $(\%)$ & For $(\%)$ & Sil (\%) & Oli/Pyr (\%) & Ens $(\%)$ & For $(\%)$ & Sil (\%) \\
\hline FQTau & M2 & $17.9_{-11.8}^{+15.3}$ & $1.0_{-0.3}^{+7.1}$ & $0.4_{-0.2}^{+3.3}$ & $5.2_{-2.7}^{+7.0}$ & $49.6_{-28.2}^{+32.6}$ & $9.1_{-4.1}^{+13.6}$ & $3.0_{-2.4}^{+8.0}$ & $4.8_{-3.0}^{+7.8}$ & $9.0_{-3.6}^{+7.0}$ & $\ldots$ & 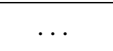 & $0.0_{-0.0}^{+2.7}$ \\
\hline FSTau & M1 & $32.5_{-9.6}^{+10.9}$ & $1.6_{-0.6}^{+3.0}$ & $0.3_{-0.0}^{+1.4}$ & $1.3_{-0.6}^{+1.7}$ & $42.6_{-14.7}^{+11.4}$ & $0.0_{-0.0}^{+2.1}$ & $0.0_{-0.0}^{+2.2}$ & $0.0_{-0.0}^{+2.5}$ & $17.0_{-6.1}^{+5.8}$ & $\ldots$ & $\ldots$ & $4.6_{-3.2}^{+5.2}$ \\
\hline FTTau & $\mathrm{c}$ & $20.1_{-3.9}^{+5.5}$ & $1.0_{-0.8}^{+1.4}$ & $2.9_{-1.5}^{+1.8}$ & $0.0_{-0.0}^{+1.0}$ & $13.9_{-3.6}^{+8.6}$ & $3.9_{-1.6}^{+3.4}$ & $2.1_{-1.4}^{+2.1}$ & $0.1_{-0.0}^{+1.6}$ & $49.7_{-27.4}^{+15.9}$ & $\ldots$ & $\ldots$ & $6.4_{-3.6}^{+10.3}$ \\
\hline$\cdots$ & & $14.4_{-0.0}^{+17.8}$ & $0.0_{-0.0}^{+10.9}$ & $0.0_{-0.0}^{+4.4}$ & $2.9_{-0.0}^{+13.7}$ & $48.8_{-5.6}^{+22.9}$ & $0.0_{-0.0}^{+23.7}$ & $2.9_{-0.0}^{+11.9}$ & $0.5_{-0.0}^{+35.3}$ & $12.0_{-0.0}^{+30.6}$ & $\ldots$ & $\cdots$ & $18.6_{-0.0}^{+15.1}$ \\
\hline FVTau & K5 & $1.4_{-0.0}^{+6.5}$ & $0.0_{-0.0}^{+4.8}$ & $3.9_{-0.0}^{+3.0}$ & $0.0_{-0.0}^{+0.8}$ & $22.7_{-0.0}^{+19.4}$ & $12.8_{-7.2}^{+8.7}$ & $9.0_{-0.0}^{+11.8}$ & $0.0_{-0.0}^{+3.0}$ & $43.2_{-13.5}^{+12.9}$ & .. & 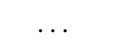 & $6.9_{-0.0}^{+16.1}$ \\
\hline FXTau & M1 & $28.2_{-5.6}^{+8.6}$ & $3.7_{-0.0}^{+5.0}$ & $3.4_{-1.6}^{+1.8}$ & $0.0_{-0.0}^{+0.8}$ & $46.5_{-13.3}^{+10.5}$ & $6.3_{-2.5}^{+4.2}$ & $0.0_{-0.0}^{+2.1}$ & $0.0_{-0.0}^{+1.6}$ & $4.0_{-0.0}^{+3.6}$ & $\ldots$ & $\ldots$ & $8.0_{-1.8}^{+2.6}$ \\
\hline .. & & $21.1_{-3.5}^{+14.8}$ & $0.0_{-0.0}^{+8.8}$ & $3.9_{-0.0}^{+8.7}$ & $4.6_{-0.0}^{+16.3}$ & $25.3_{-8.6}^{+16.6}$ & $0.2_{-0.0}^{+2.7}$ & $0.2_{-0.0}^{+4.2}$ & $1.7_{-0.0}^{+10.7}$ & $33.3_{-14.1}^{+16.6}$ & $\ldots$ & $\ldots$ & $9.6_{-0.0}^{+21.9}$ \\
\hline FZTau & M0 & $24.8_{-10.9}^{+7.5}$ & $1.3_{-0.0}^{+2.2}$ & $1.9_{-0.9}^{+1.0}$ & $10.7_{-2.6}^{+2.6}$ & $19.1_{-5.4}^{+10.8}$ & $4.4_{-1.9}^{+2.3}$ & $1.5_{-0.5}^{+2.2}$ & $0.0_{-0.0}^{+2.8}$ & $25.0_{-8.4}^{+6.4}$ & $\ldots$ & $\ldots$ & $11.1_{-4.2}^{+4.0}$ \\
\hline GGTau & M0 & $9.2_{-3.7}^{+3.4}$ & $0.9_{-0.0}^{+2.0}$ & $1.6_{-0.0}^{+1.4}$ & $0.0_{-0.0}^{+0.9}$ & $54.2_{-15.3}^{+15.5}$ & $0.0_{-0.0}^{+2.1}$ & $1.9_{-1.3}^{+2.8}$ & $0.0_{-0.0}^{+1.1}$ & $16.5_{-3.8}^{+4.7}$ & $\ldots$ & $\ldots$ & $15.7_{-4.4}^{+4.9}$ \\
\hline GHTau & M2 & $10.6_{-3.9}^{+3.1}$ & $1.7_{-0.9}^{+1.7}$ & $2.1_{-1.3}^{+1.1}$ & $0.7_{-0.7}^{+1.4}$ & $27.3_{-11.6}^{+15.0}$ & $7.3_{-4.0}^{+3.2}$ & $0.8_{-0.8}^{+2.7}$ & $4.1_{-2.9}^{+3.1}$ & $45.4_{-14.6}^{+26.6}$ & $\ldots$ & $\cdots$ & $0.0_{-0.0}^{+4.3}$ \\
\hline$\cdots$ & & $8.7_{-4.5}^{+4.5}$ & $2.0_{-2.4}^{+4.1}$ & $4.9_{-4.4}^{+5.4}$ & $2.7_{-2.3}^{+2.7}$ & $11.5_{-6.5}^{+8.2}$ & $4.4_{-3.2}^{+4.1}$ & $1.0_{-1.0}^{+1.8}$ & $12.6_{-8.0}^{+10.3}$ & $32.4_{-14.5}^{+19.8}$ & $\ldots$ & . & $19.7_{-13.8}^{+18.4}$ \\
\hline GITau & K6 & $35.6_{-10.5}^{+10.0}$ & $0.4_{-0.0}^{+3.0}$ & $1.4_{-0.9}^{+1.3}$ & $0.0_{-0.0}^{+0.6}$ & $39.3_{-7.7}^{+14.0}$ & $5.7_{-2.7}^{+2.6}$ & $1.6_{-1.0}^{+2.0}$ & $1.0_{-0.0}^{+1.8}$ & $6.4_{-1.8}^{+1.8}$ & $\ldots$ & $\ldots$ & $8.4_{-2.1}^{+2.9}$ \\
\hline$\ldots$ & & $16.0_{-7.3}^{+10.5}$ & $8.4_{-3.8}^{+5.7}$ & $11.1_{-5.2}^{+6.0}$ & $1.9_{-1.9}^{+5.1}$ & $20.5_{-7.6}^{+10.5}$ & $0.0_{-0.0}^{+2.1}$ & $0.0_{-0.0}^{+2.7}$ & $0.1_{-0.0}^{+2.8}$ & $33.9_{-12.7}^{+14.4}$ & $\ldots$ & $\ldots$ & $8.1_{-4.9}^{+9.2}$ \\
\hline GKTau & K7 & $64.5_{-10.7}^{+7.3}$ & $0.0_{-0.0}^{+0.9}$ & $3.1_{-1.3}^{+1.5}$ & $0.0_{-0.0}^{+0.7}$ & $18.0_{-6.6}^{+3.5}$ & $3.7_{-1.4}^{+0.8}$ & $0.0_{-0.0}^{+0.7}$ & $1.7_{-0.7}^{+0.9}$ & $7.3_{-1.8}^{+1.8}$ & $\ldots$ & $\ldots$ & $1.8_{-0.8}^{+0.8}$ \\
\hline GMAur & K3 & $37.3_{-11.2}^{+9.4}$ & $0.0_{-0.0}^{+3.2}$ & $3.1_{-0.0}^{+2.8}$ & $0.0_{-0.0}^{+1.3}$ & $16.0_{-4.1}^{+5.5}$ & $12.2_{-3.5}^{+3.4}$ & $14.9_{-4.2}^{+5.6}$ & $0.0_{-0.0}^{+1.2}$ & $1.2_{-0.0}^{+10.8}$ & $\ldots$ & $\ldots$ & $15.4_{-6.7}^{+4.5}$ \\
\hline$\ldots$ & & $15.7_{-0.0}^{+29.0}$ & $0.7_{-0.0}^{+5.5}$ & $0.4_{-0.0}^{+29.4}$ & $17.3_{-0.0}^{+33.6}$ & $9.6_{-0.0}^{+14.1}$ & $3.8_{-0.0}^{+20.4}$ & $0.0_{-0.0}^{+8.6}$ & $17.4_{-0.0}^{+17.6}$ & $33.6_{-0.0}^{+32.2}$ & $\cdots$ & $\cdots$ & $1.3_{-0.0}^{+14.1}$ \\
\hline GOTau & M0 & $12.0_{-7.6}^{+6.5}$ & $0.0_{-0.0}^{+4.8}$ & $3.4_{-2.3}^{+2.8}$ & $0.1_{-0.0}^{+2.0}$ & $63.2_{-37.8}^{+24.7}$ & $3.3_{-4.1}^{+6.0}$ & $0.0_{-1.2}^{+5.4}$ & $5.6_{-4.8}^{+7.8}$ & $12.4_{-5.3}^{+6.4}$ & $\ldots$ & $\ldots$ & $0.0_{-0.2}^{+2.0}$ \\
\hline HKTau & M1 & $1.0_{-0.3}^{+2.9}$ & $0.4_{-0.0}^{+2.0}$ & $1.8_{-0.0}^{+1.3}$ & $0.1_{-0.0}^{+1.1}$ & $37.5_{-21.5}^{+6.1}$ & $7.7_{-4.7}^{+2.8}$ & $8.2_{-2.8}^{+4.0}$ & $0.0_{-0.0}^{+3.3}$ & $18.6_{-6.0}^{+8.1}$ & $\ldots$ & $\ldots$ & $24.8_{-11.5}^{+4.5}$ \\
\hline$\cdots$ & & $19.5_{-3.4}^{+29.6}$ & $5.3_{-0.0}^{+10.3}$ & $1.3_{-0.0}^{+8.8}$ & $11.1_{-2.5}^{+54.3}$ & $13.5_{-3.4}^{+33.1}$ & $6.7_{-0.0}^{+33.1}$ & $2.9_{-0.0}^{+5.6}$ & $7.4_{-0.0}^{+64.7}$ & $21.5_{-6.3}^{+25.3}$ & $\ldots$ & $\ldots$ & $10.6_{-6.4}^{+14.0}$ \\
\hline HNTau & K5 & $13.9_{-3.7}^{+6.7}$ & $0.0_{-0.0}^{+0.4}$ & $0.8_{-0.0}^{+0.7}$ & $0.0_{-0.0}^{+0.4}$ & $45.5_{-7.7}^{+12.1}$ & $0.0_{-0.0}^{+1.3}$ & $0.0_{-0.0}^{+1.3}$ & $0.0_{-0.0}^{+1.3}$ & $22.9_{-4.6}^{+4.8}$ & $\ldots$ & $\ldots$ & $16.8_{-3.2}^{+4.5}$ \\
\hline HOTau & M0.5 & $10.8_{-1.6}^{+3.7}$ & $0.7_{-0.4}^{+1.0}$ & $2.0_{-0.6}^{+2.6}$ & $0.0_{-0.0}^{+0.7}$ & $34.6_{-8.6}^{+12.9}$ & $6.2_{-3.9}^{+3.6}$ & $2.2_{-0.0}^{+5.5}$ & $0.4_{-0.0}^{+2.2}$ & $43.0_{-17.1}^{+11.7}$ & $\cdots$ & $\cdots$ & $0.0_{-0.0}^{+10.3}$ \\
\hline$\cdots$ & & $38.3_{-16.0}^{+19.0}$ & $0.0_{-0.0}^{+1.2}$ & $1.6_{-0.0}^{+4.0}$ & $0.0_{-0.0}^{+2.7}$ & $37.6_{-11.3}^{+23.2}$ & $0.4_{-0.0}^{+5.9}$ & $0.3_{-0.0}^{+2.0}$ & $4.2_{-3.4}^{+7.1}$ & $8.3_{-3.6}^{+7.0}$ & $\cdots$ & $\cdots$ & $9.3_{-5.8}^{+3.2}$ \\
\hline HРТаu & K3 & $57.4_{-10.6}^{+10.9}$ & $0.0_{-0.0}^{+0.8}$ & $1.0_{-0.0}^{+1.0}$ & $0.0_{-0.0}^{+0.9}$ & $22.1_{-4.9}^{+3.6}$ & $1.1_{-0.0}^{+1.9}$ & $0.0_{-0.0}^{+1.3}$ & $0.9_{-0.0}^{+1.3}$ & $14.3_{-2.3}^{+4.4}$ & $\ldots$ & $\ldots$ & $3.4_{-1.3}^{+1.3}$ \\
\hline Haro6-37 & K7 & $11.4_{-7.7}^{+13.5}$ & $0.0_{-0.0}^{+1.6}$ & $3.4_{-1.8}^{+2.2}$ & $3.0_{-1.8}^{+2.9}$ & $45.0_{-27.5}^{+16.2}$ & $10.4_{-5.4}^{+5.8}$ & $2.6_{-0.0}^{+4.5}$ & $7.0_{-4.0}^{+6.5}$ & $17.2_{-7.0}^{+12.2}$ & $\ldots$ & $\ldots$ & $0.0_{-0.0}^{+7.1}$ \\
\hline IPTau & M0 & $28.0_{-7.4}^{+9.2}$ & $0.8_{-0.3}^{+2.4}$ & $2.5_{-1.7}^{+1.4}$ & $0.0_{-0.0}^{+0.5}$ & $33.0_{-7.5}^{+9.9}$ & $4.5_{-2.4}^{+4.4}$ & $3.9_{-2.5}^{+5.0}$ & $0.0_{-0.0}^{+1.2}$ & $18.7_{-8.3}^{+12.5}$ & $\ldots$ & $\ldots$ & $8.5_{-4.1}^{+3.2}$ \\
\hline$\ldots$ & & $17.0_{-6.0}^{+8.1}$ & $0.2_{-0.0}^{+7.4}$ & $1.7_{-0.1}^{+5.7}$ & $8.2_{-2.9}^{+5.6}$ & $19.3_{-8.9}^{+18.2}$ & $4.2_{-0.0}^{+18.2}$ & $1.8_{-0.0}^{+9.0}$ & $13.7_{-5.7}^{+8.5}$ & $32.3_{-6.9}^{+11.1}$ & $\ldots$ & $\ldots$ & $1.7_{-1.4}^{+12.0}$ \\
\hline IQTau & M0.5 & $17.3_{-4.0}^{+4.1}$ & $0.0_{-0.0}^{+1.4}$ & $2.0_{-1.1}^{+1.7}$ & $0.0_{-0.0}^{+0.9}$ & $30.7_{-8.7}^{+8.8}$ & $8.4_{-2.4}^{+3.6}$ & $2.7_{-1.4}^{+3.5}$ & $1.6_{-0.9}^{+2.4}$ & $28.7_{-11.3}^{+6.8}$ & $\ldots$ & $\cdots$ & $8.7_{-3.8}^{+3.9}$ \\
\hline$\ldots$ & & $24.3_{-6.3}^{+8.9}$ & $0.0_{-0.0}^{+1.2}$ & $0.7_{-0.4}^{+2.7}$ & $1.6_{-1.0}^{+3.1}$ & $43.9_{-12.9}^{+14.6}$ & $0.0_{-0.0}^{+4.6}$ & $0.6_{-0.0}^{+3.1}$ & $0.0_{-0.0}^{+6.5}$ & $14.7_{-3.3}^{+9.1}$ & $\ldots$ & $\ldots$ & $14.1_{-7.1}^{+12.4}$ \\
\hline ISTau & K7 & $0.0_{-0.0}^{+4.1}$ & $0.0_{-0.0}^{+3.3}$ & $6.8_{-1.8}^{+2.9}$ & $11.6_{-3.3}^{+3.8}$ & $47.8_{-16.1}^{+13.5}$ & $19.0_{-5.2}^{+7.3}$ & $5.1_{-4.2}^{+7.1}$ & $0.3_{-0.0}^{+4.4}$ & $9.4_{-3.0}^{+8.4}$ & $\ldots$ & $\ldots$ & $0.0_{-0.0}^{+7.7}$ \\
\hline$\cdots$ & & $3.0_{-0.0}^{+24.8}$ & $0.0_{-0.0}^{+31.7}$ & $10.4_{-0.0}^{+55.5}$ & $6.8_{-0.0}^{+66.0}$ & $26.5_{-13.3}^{+42.8}$ & $0.0_{-0.0}^{+76.9}$ & $14.0_{-0.0}^{+97.4}$ & $4.7_{-0.0}^{+66.6}$ & $26.9_{-6.9}^{+67.9}$ & $\ldots$ & $\ldots$ & $7.6_{-0.0}^{+47.0}$ \\
\hline LkCa15 & K5 & $55.9_{-15.1}^{+11.0}$ & $0.0_{-0.0}^{+2.0}$ & $2.7_{-0.0}^{+3.8}$ & $0.0_{-0.0}^{+0.6}$ & $21.6_{-6.1}^{+11.5}$ & $7.7_{-2.7}^{+2.7}$ & $6.3_{-3.0}^{+3.5}$ & $0.0_{-0.0}^{+0.6}$ & $4.8_{-0.0}^{+2.7}$ & $\ldots$ & $\ldots$ & $0.8_{-0.0}^{+3.1}$ \\
\hline$\cdots$ & & $22.0_{-8.4}^{+11.7}$ & $9.2_{-6.1}^{+6.1}$ & $0.0_{-0.0}^{+2.0}$ & $10.2_{-5.8}^{+7.4}$ & $16.0_{-6.8}^{+7.7}$ & $6.1_{-3.6}^{+5.6}$ & $0.0_{-0.4}^{+1.5}$ & $6.6_{-4.9}^{+6.3}$ & $16.2_{-6.9}^{+8.1}$ & $\ldots$ & $\cdots$ & $13.7_{-7.0}^{+9.5}$ \\
\hline RWAur & K3 & $26.1_{-9.6}^{+9.0}$ & $0.3_{-0.0}^{+1.0}$ & $1.5_{-0.0}^{+2.1}$ & $0.0_{-0.0}^{+0.5}$ & $39.0_{-23.1}^{+9.1}$ & $2.3_{-0.0}^{+3.9}$ & $0.0_{-0.0}^{+1.8}$ & $0.0_{-0.0}^{+1.5}$ & $12.4_{-8.7}^{+3.3}$ & $\ldots$ & $\cdots$ & $18.3_{-6.8}^{+6.7}$ \\
\hline RYTau & G1 & $6.5_{-1.8}^{+3.8}$ & $8.4_{-2.7}^{+1.7}$ & $1.1_{-0.6}^{+0.8}$ & $8.0_{-1.5}^{+1.7}$ & $69.7_{-10.2}^{+13.3}$ & $1.9_{-0.0}^{+3.3}$ & $0.0_{-0.0}^{+1.6}$ & $1.0_{-0.0}^{+2.1}$ & $3.3_{-1.1}^{+1.8}$ & $\cdots$ & $\ldots$ & $0.0_{-0.0}^{+0.2}$ \\
\hline SUAur & G1 & $20.2_{-5.4}^{+5.0}$ & $0.0_{-0.0}^{+0.6}$ & $1.3_{-0.9}^{+1.0}$ & $0.0_{-0.0}^{+0.6}$ & $49.7_{-8.5}^{+8.5}$ & $1.2_{-0.0}^{+1.8}$ & $2.4_{-0.0}^{+3.3}$ & $0.0_{-0.0}^{+0.6}$ & $3.9_{-1.7}^{+1.9}$ & $\ldots$ & $\ldots$ & $21.3_{-3.6}^{+3.1}$ \\
\hline UYAur & K7 & $19.9_{-6.8}^{+6.4}$ & $0.1_{-0.0}^{+2.6}$ & $0.0_{-0.0}^{+1.3}$ & $0.0_{-0.0}^{+1.9}$ & $54.3_{-13.1}^{+11.1}$ & $0.0_{-0.0}^{+1.9}$ & $1.3_{-0.0}^{+3.6}$ & $0.0_{-0.0}^{+1.3}$ & $14.1_{-6.2}^{+3.9}$ & $\ldots$ & $\ldots$ & $10.4_{-0.0}^{+7.2}$ \\
\hline V710Tau & M1 & $27.7_{-19.2}^{+14.5}$ & $0.0_{-0.0}^{+1.6}$ & $2.8_{-1.8}^{+2.6}$ & $0.9_{-0.6}^{+1.45}$ & $39.1_{-28.1}^{+17.8}$ & $8.5_{-5.0}^{+6.9}$ & $6.0_{-3.5}^{+5.9}$ & $8.0_{-4.6}^{+6.2}$ & $3.7_{-1.8}^{+1.9}$ & $\ldots$ & $\cdots$ & $3.3_{-1.3}^{+2.5}$ \\
\hline V773Tau & K3 & $26.2_{-5.4}^{+24.6}$ & $3.8_{-0.0}^{+5.9}$ & $1.3_{-0.0}^{+2.3}$ & $0.0_{-0.0}^{+1.8}$ & $40.9_{-17.2}^{+12.7}$ & $4.3_{-0.0}^{+9.4}$ & $0.0_{-0.0}^{+1.8}$ & $0.0_{-0.0}^{+8.0}$ & $10.9_{-3.8}^{+5.7}$ & $\ldots$ & $\ldots$ & $12.5_{-6.3}^{+5.6}$ \\
\hline V836Tau & K7 & $1.2_{-0.0}^{+3.4}$ & $0.9_{-0.6}^{+0.8}$ & $4.5_{-0.8}^{+1.9}$ & $0.0_{-0.0}^{+0.9}$ & $32.4_{-14.5}^{+16.9}$ & $9.1_{-3.3}^{+4.2}$ & $2.8_{-1.9}^{+3.4}$ & $3.9_{-2.8}^{+2.1}$ & $45.1_{-35.6}^{+9.7}$ & $\ldots$ & $\ldots$ & $0.0_{-0.0}^{+8.7}$ \\
\hline$\ldots$ & & $30.7_{-8.9}^{+48.4}$ & $0.0_{-0.0}^{+26.6}$ & $1.7_{-0.0}^{+9.2}$ & $10.4_{-0.0}^{+61.5}$ & $32.6_{-19.6}^{+52.5}$ & $3.9_{-0.0}^{+23.7}$ & $1.7_{-0.0}^{+12.2}$ & $0.5_{-0.0}^{+24.0}$ & $17.0_{-3.8}^{+30.6}$ & $\cdots$ & $\ldots$ & $1.5_{-1.5}^{+3.7}$ \\
\hline V955Tau & K5 & $0.0_{-0.0}^{+1.3}$ & $0.0_{-0.0}^{+0.9}$ & $6.3_{-1.6}^{+2.5}$ & $10.7_{-2.8}^{+3.1}$ & $59.3_{-32.6}^{+15.0}$ & $4.6_{-2.1}^{+5.8}$ & $0.0_{-0.0}^{+3.0}$ & $0.0_{-0.1}^{+2.9}$ & $11.9_{-3.6}^{+4.7}$ & $\ldots$ & $\ldots$ & $7.2_{-1.8}^{+6.7}$ \\
\hline VYTau & M0 & $2.5_{-0.0}^{+13.5}$ & $0.0_{-0.0}^{+12.4}$ & $3.3_{-0.0}^{+16.1}$ & $0.0_{-0.0}^{+8.4}$ & $18.1_{-8.2}^{+4.7}$ & $1.1_{-0.1}^{+7.2}$ & $0.0_{-0.0}^{+14.7}$ & $0.6_{-0.0}^{+7.6}$ & $74.0_{-47.6}^{+14.1}$ & $\ldots$ & $\ldots$ & $0.3_{-0.0}^{+18.0}$ \\
\hline ZZTauIRS & M4.5 & $16.0_{-3.5}^{+6.3}$ & $0.7_{-0.0}^{+3.0}$ & $0.7_{-0.0}^{+2.2}$ & $0.1_{-0.0}^{+1.2}$ & $76.1_{-21.8}^{+12.9}$ & $0.3_{-0.5}^{+4.3}$ & $3.3_{-1.9}^{+4.8}$ & $0.0_{-0.0}^{+2.9}$ & $0.0_{-0.0}^{+1.6}$ & & $\cdots$ & $2.8_{-0.0}^{+7.3}$ \\
\hline$\cdots$ & & $16.1_{-6.5}^{+8.6}$ & $0.0_{-0.2}^{+2.0}$ & $8.1_{-5.0}^{+6.9}$ & $6.0_{-3.3}^{+4.7}$ & $39.4_{-15.4}^{+18.7}$ & $0.0_{-0.4}^{+3.7}$ & $9.1_{-5.2}^{+7.5}$ & $9.7_{-6.1}^{+9.3}$ & $7.0_{-3.5}^{+5.2}$ & $\cdots$ & $\cdots$ & $4.6_{-2.9}^{+4.3}$ \\
\hline \multicolumn{14}{|c|}{ Upper Scorpius } \\
\hline PBB2002 J160357.9 & M2 & $8.2_{-2.1}^{+16.3}$ & $0.0_{-0.0}^{+1.5}$ & $2.1_{-0.2}^{+4.6}$ & $0.0_{-0.0}^{+1.0}$ & $42.9_{-24.5}^{+14.8}$ & $0.0_{-4.5}^{+0.5}$ & $0.0_{-0.0}^{+4.7}$ & $0.0_{-0.0}^{+3.9}$ & $46.8_{-32.4}^{+28.8}$ & 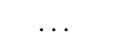 & $\because$ & $0.0_{-0.0}^{+10.9}$ \\
\hline PBB2002 J160823.2 & K9 & $2.4_{-0.0}^{+6.2}$ & $0.0_{-0.0}^{+5.3}$ & $3.8_{-0.0}^{+6.2}$ & $0.0_{-0.0}^{+1.1}$ & $27.1_{-24.1}^{+2.5}$ & $5.9_{-3.6}^{+3.9}$ & $0.0_{-0.0}^{+7.7}$ & $0.6_{-0.0}^{+10.4}$ & $60.1_{-22.7}^{+38.8}$ & $\ldots$ & $\ldots$ & $0.0_{-0.1}^{+5.1}$ \\
\hline PBB2002 J160900.7 & K9 & $17.2_{-4.5}^{+6.5}$ & $0.5_{-0.0}^{+1.4}$ & $3.0_{-1.6}^{+2.0}$ & $0.0_{-0.0}^{+0.3}$ & $42.3_{-17.3}^{+14.3}$ & $4.1_{-2.4}^{+3.7}$ & $0.0_{-0.0}^{+2.5}$ & $0.0_{-0.0}^{+1.0}$ & $24.9_{-16.8}^{+2.9}$ & $\cdots$ & $\cdots$ & $8.0_{-4.0}^{+4.9}$ \\
\hline PBB2002 J160959.4 & M4 & $17.7_{-11.4}^{+31.6}$ & $0.0_{-0.0}^{+2.7}$ & $4.8_{-2.4}^{+3.8}$ & $0.5_{-0.0}^{+2.5}$ & $61.9_{-50.2}^{+16.5}$ & $10.6_{-8.1}^{+12.0}$ & $0.0_{-0.0}^{+7.6}$ & $0.0_{-0.0}^{+4.5}$ & $3.1_{-0.9}^{+5.9}$ & $\ldots$ & $\ldots$ & $1.2_{-0.2}^{+11.7}$ \\
\hline PBB2002 J161115.3 & M1 & $20.3_{-12.7}^{+4.2}$ & $0.0_{-0.0}^{+2.4}$ & $1.9_{-1.4}^{+2.5}$ & $0.0_{-0.0}^{+0.7}$ & $58.7_{-26.8}^{+11.1}$ & $3.1_{-2.0}^{+5.0}$ & $0.0_{-0.0}^{+6.7}$ & $1.5_{-0.4}^{+2.6}$ & $9.2_{-4.6}^{+3.2}$ & . & $\ldots$ & $5.3_{-4.9}^{+1.3}$ \\
\hline PBB2002 J161420.2 & M0 & $6.5_{-1.4}^{+1.4}$ & $0.0_{-0.0}^{+0.5}$ & $1.4_{-0.9}^{+0.9}$ & $0.0_{-0.0}^{+0.3}$ & $67.7_{-7.8}^{+7.9}$ & $0.6_{-0.0}^{+0.9}$ & $0.8_{-0.0}^{+1.2}$ & $0.0_{-0.0}^{+0.4}$ & $15.2_{-1.5}^{+2.8}$ & $\cdots$ & $\cdots$ & $7.9_{-1.3}^{+1.3}$ \\
\hline PZ99 J160357.6 & K5 & $0.0_{-0.0}^{+3.1}$ & $0.0_{-0.0}^{+2.8}$ & $2.8_{-0.0}^{+5.1}$ & $0.0_{-0.0}^{+3.2}$ & $6.1_{-0.0}^{+9.2}$ & $3.8_{-0.0}^{+2.9}$ & $0.2_{-0.0}^{+2.2}$ & $0.0_{-0.0}^{+2.6}$ & $87.1_{-46.7}^{+19.9}$ & $\ldots$ & $\cdots$ & $0.0_{-0.0}^{+5.1}$ \\
\hline
\end{tabular}


Table 5

(Continued)

\begin{tabular}{|c|c|c|c|c|c|c|c|c|c|c|c|c|c|}
\hline \multirow[t]{2}{*}{ ID } & \multirow[t]{2}{*}{$\mathrm{SpT}$} & \multicolumn{4}{|c|}{$0.1(\mu \mathrm{m})$} & \multicolumn{4}{|c|}{$1.5(\mu \mathrm{m})$} & \multicolumn{4}{|c|}{$6.0(\mu \mathrm{m})$} \\
\hline & & $\mathrm{Oli} / \mathrm{Pyr}^{\mathrm{b}}(\%)$ & Ens $(\%)$ & For $(\%)$ & Sil $(\%)$ & Oli/Pyr (\%) & Ens $(\%)$ & For $(\%)$ & $\operatorname{Sil}(\%)$ & Oli/Pyr (\%) & Ens $(\%)$ & For $(\%)$ & $\operatorname{Sil}(\%)$ \\
\hline PZ99 J161411.0 & K0 & $0.0_{-0.0}^{+2.0}$ & $0.0_{-0.0}^{+4.0}$ & $1.1_{-0.0}^{+2.1}$ & $1.4_{-0.0}^{+4.4}$ & $8.8_{-0.0}^{+4.7}$ & $3.9_{-0.0}^{+3.5}$ & $0.0_{-0.0}^{+1.4}$ & $0.0_{-0.0}^{+4.1}$ & $84.8_{-55.2}^{+8.6}$ & $\ldots$ & $\ldots$ & $0.0_{-0.0}^{+3.0}$ \\
\hline ScoPMS31 & M0.5 & $9.6_{-11.3}^{+7.5}$ & $\begin{array}{l}0.0_{-0.0}^{+2.5} \\
\end{array}$ & $\begin{array}{r}1.9_{-1.8}^{+3.3} \\
\end{array}$ & $\begin{array}{r}3.0_{-1.6}^{+4.6} \\
\end{array}$ & $\begin{array}{r}72.4_{-61.0}^{+39.8} \\
\end{array}$ & $4.6_{-3.7}^{+10.7}$ & $0.0_{-1.9}^{+9.0}$ & $\begin{array}{r}1.9_{-1.4}^{+9.2} \\
\end{array}$ & $6.6_{-6.2}^{+4.2}$ & $\cdots$ & $\cdots$ & $\begin{array}{r}0.0_{-0.0}^{+1.5} \\
\end{array}$ \\
\hline \multicolumn{14}{|c|}{ Eta Chamaeleontis } \\
\hline J0843 & M3.4 & $27.2_{-11.0}^{+16.9}$ & $1.1_{-0.0}^{+8.3}$ & $4.4_{-0.0}^{+3.3}$ & $1.5_{-0.0}^{+2.8}$ & $44.6_{-21.9}^{+19.9}$ & $5.9_{-0.0}^{+7.6}$ & $0.0_{-0.0}^{+10.2}$ & $1.6_{-0.0}^{+11.6}$ & $13.6_{-0.0}^{+19.2}$ & $\cdots$ & $\cdots$ & $0.0_{-0.0}^{+3.9}$ \\
\hline RECX-5 & M3.8 & $36.1_{-19.4}^{+23.4}$ & $0.0_{-0.0}^{+2.9}$ & $12.3_{-0.0}^{+8.7}$ & $0.3_{-0.0}^{+14.8}$ & $12.3_{-3.5}^{+15.6}$ & $23.9_{-0.0}^{+13.9}$ & $0.0_{-0.0}^{+8.0}$ & $8.0_{-3.3}^{+6.9}$ & $7.1_{-0.0}^{+18.9}$ & . & $\cdots$ & $0.0_{-0.0}^{+10.0}$ \\
\hline RECX-9 & M4.4 & $42.9_{-34.1}^{+21.6}$ & $0.0_{-0.0}^{+6.0}$ & $1.9_{-1.0}^{+3.9}$ & $8.6_{-6.7}^{+8.3}$ & $31.6_{-24.2}^{+15.8}$ & $0.9_{-0.0}^{+7.0}$ & $5.4_{-3.2}^{+5.8}$ & $4.6_{-2.1}^{+10.8}$ & $3.3_{-1.1}^{+3.0}$ & $\cdots$ & $\cdots$ & $0.8_{-0.0}^{+8.3}$ \\
\hline RECX-11 & K6.5 & $36.9_{-17.2}^{+18.1}$ & $1.4_{-0.0}^{+3.4}$ & $3.4_{-0.0}^{+8.4}$ & $2.6_{-0.0}^{+4.2}$ & $26.8_{-14.4}^{+15.7}$ & $8.0_{-0.0}^{+10.5}$ & $0.0_{-0.0}^{+2.7}$ & $3.4_{-1.9}^{+3.7}$ & $17.6_{-6.6}^{+8.1}$ & $\ldots$ & $\ldots$ & $0.0_{-0.0}^{+1.9}$ \\
\hline
\end{tabular}

Notes.

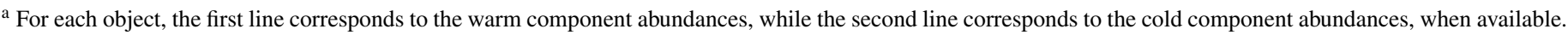

b Amorphous olivine and pyroxene combined.

derived for 139 YSOs belonging to four young star clusters using the same method.

Serpens and Taurus are used as prototypes of young regions, where most stars are still surrounded by disks, while Upper Sco and $\eta$ Cha represent the older bin of disk evolution, where a large fraction of the disks have already dissipated but some massive protoplanetary disks are left. The large number of objects analyzed allows statistical results that point to the main processes that affect the grain size distribution and composition of dust in protoplanetary disks. Furthermore, the usage of the same analysis method for regions of different mean ages allows a study of evolution of the dust parameters with time.

Our large sample does not show a preferential grain size or crystallinity fraction with disk geometry, contrary to earlier analyses based on smaller samples. Also, younger and older regions have very similar distributions. The difference between mean mass-averaged grain sizes for the warm and cold components of a given star-forming region is small, however a considerable difference is seen between the ranges of grain sizes spanned in both components. The cold mass-averaged grain sizes never reach the largest size modeled $(6 \mu \mathrm{m})$ while the warm massaveraged grain sizes span the entire range of sizes modeled. The crystallinity fractions derived for inner (warm) and outer (cold) disks are typically $10 \%-20 \%$, and not correlated. The cold crystallinity fraction shows a larger spread than the warm. No strong difference is seen between the overall mean warm and cold crystallinity fraction. Within the crystalline dust population, more enstatite is found in the warm component and more forsterite in the cold component. The differences are not very significant, however.

The results of the spectral decomposition support the usage of the strength of the $10 \mu \mathrm{m}$ silicate feature $\left(S_{\text {peak }}^{10 \mu \mathrm{m}}\right)$ as a proxy of the mean grain size of dust in the disk surface. This is supported by the correlation between $S_{\text {peak }}^{10 \mu \mathrm{m}}$ and mean grain size and lack of correlation with the mean crystallinity.

Mean cluster ages and disk fractions are used as indicators of the evolutionary stage of the different populations. Our results show that the different regions have similar distributions of mean grain sizes and crystallinity fractions regardless of the spread in mean ages of 1-8 Myr. Thus, despite the fact that the majority of disks dissipate within a few Myr, the surface dust properties do not depend on age for those disks that have not yet dissipated in the 1-8 Myr range. This points to a rapid change in the composition and crystallinity of the dust in the early stages $(\leqslant 1 \mathrm{Myr})$ that is maintained essentially until the disks dissipate.
Astrochemistry at Leiden is supported by a Spinoza grant from the Netherlands Organization for Scientific Research (NWO) and by the Netherlands Research School for Astronomy (NOVA) grants. This work is based on observations made with the Spitzer Space Telescope, which is operated by the Jet Propulsion Laboratory, California Institute of Technology under a contract with NASA. Support for this work was provided by NASA through an award issued by JPL/Caltech.

\section{APPENDIX}

\section{RELATIVE ABUNDANCES OF SPECIES}

The relative abundances, as resulting from the $\mathrm{B} 2 \mathrm{C}$ compositional fitting (Section 3) to the IRS spectra of protoplanetary disks in Serpens, Taurus, Upper Sco, and $\eta$ Cha, are shown in Table 5. Since the opacities of amorphous olivine and pyroxene are degenerate, the abundances of these two species have been added into one, marked "Oli/Pyr" in the table. Furthermore, "Sil" designates the amorphous silica, and the crystalline enstatite and forsterite are marked as "Ens" and "For," respectively. In the table, the first line of a given object corresponds to the results of the fit to the warm component and the second line to the results of the cold component. For some objects (20 in Serpens, 28 in Taurus, all in Upper Sco and $\eta$ Cha), the $\mathrm{S} / \mathrm{N}$ drops considerably at longer wavelengths and the results of the procedure are no longer reliable. For these sources, the cold component could not be fitted satisfactorily and, in Table 5, only the warm component results are shown.

\section{REFERENCES}

Ábrahám, P., et al. 2009, Nature, 459, 224

Apai, D., Pascucci, I., Bouwman, J., Natta, A., Henning, T., \& Dullemond, C. P. 2005, Science, 310,834

Beckwith, S. V. W., Henning, T., \& Nakagawa, Y. 2000, in Protostars and Planets IV, ed. V. Mannings, A. P. Boss, \& S. S. Russell (Tucson, AZ: Univ. Arizona Press), 533

Beichman, C. A., et al. 2006, ApJ, 639, 1166

Blaauw, A. 1978, in Problems of Physics and Evolution of the Universe, ed. L. V. Mirzoyan (Yerevan: Publishing House of the Armenian Academy of Sciences), 101

Bockelée-Morvan, D., et al. 2000, A\&A, 353, 1101

Bouwman, J., Meeus, G., de Koter, A., Hony, S., Dominik, C., \& Waters, L. B. F. M. 2001, A\&A, 375, 950

Bouwman, J., et al. 2008, ApJ, 683, 479

Bouy, H., et al. 2008, A\&A, 486, 877

Brown, J. M., et al. 2007, ApJ, 664, L107

Bryden, G., et al. 2006, ApJ, 636, 1098 
Carpenter, J. M., Mamajek, E. E., Hillenbrand, L. A., \& Meyer, M. R. 2006, ApJ, 651, L49

Carpenter, J. M., et al. 2009, ApJS, 181, 197

Ciesla, F. J. 2007, Science, 318, 613

Ciesla, F. J. 2009, Icarus, 200, 655

Crovisier, J., Leech, K., Bockelee-Morvan, D., Brooke, T. Y., Hanner, M. S., Altieri, B., Keller, H. U., \& Lellouch, E. 1997, Science, 275, 1904

Dahm, S. E., \& Carpenter, J. M. 2009, AJ, 137, 4024

Desch, S. J., \& Connolly, H. C., Jr. 2002, Meteorit. Planet. Sci., 37, 183

de Zeeuw, P. T., Hoogerwerf, R., de Bruijne, J. H. J., Brown, A. G. A., \& Blaauw, A. 1999, AJ, 117,354

Dullemond, C. P., \& Dominik, C. 2005, A\&A, 434, 971

Dullemond, C. P., \& Dominik, C. 2008, A\&A, 487, 205

Dullemond, C. P., Dominik, C., \& Natta, A. 2001, ApJ, 560, 957

Dzib, S., Loinard, L., Mioduszewski, A. J., Boden, A. F., Rodríguez, L. F., \& Torres, R. M. 2010, ApJ, 718, 610

Fabian, D., Jäger, C., Henning, T., Dorschner, J., \& Mutschke, H. 2000, A\&A, 364,282

Forrest, W. J., et al. 2004, ApJS, 154, 443

Furlan, E., et al. 2006, ApJS, 165, 568

Gail, H.-P. 2004, A\&A, 413, 571

Gautier, T. N., III., et al. 2007, ApJ, 667, 527

Geers, V. C., et al. 2006, A\&A, 459, 545

Haisch, K. E., Jr., Lada, E. A., \& Lada, C. J. 2001, ApJ, 553, L153

Harker, D. E., \& Desch, S. J. 2002, ApJ, 565, L109

Harker, D. E., Wooden, D. H., Woodward, C. E., \& Lisse, C. M. 2002, ApJ, 580,579

Harker, D. E., Woodward, C. E., \& Wooden, D. H. 2005, Science, 310, 278

Harker, D. E., Woodward, C. E., Wooden, D. H., Fisher, R. S., \& Trujillo, C. A. 2007, Icarus, 190, 432

Hartmann, L., Ballesteros-Paredes, J., \& Bergin, E. A. 2001, ApJ, 562, 852

Henning, T. 2010, ARA\&A, 48, 21

Hernández, J., Hartmann, L., Calvet, N., Jeffries, R. D., Gutermuth, R., Muzerolle, J., \& Stauffer, J. 2008, ApJ, 686, 1195

Houck, J. R., et al. 2004, ApJS, 154, 18

Juhász, A., Henning, T., Bouwman, J., Dullemond, C. P., Pascucci, I., \& Apai, D. 2009, ApJ, 695, 1024

Juhász, A., et al. 2010, ApJ, 721, 431

Kalas, P., et al. 2008, Science, 322, 1345

Kemper, F., Vriend, W. J., \& Tielens, A. G. G. M. 2004, ApJ, 609, 826

Kenyon, S. J., Dobrzycka, D., \& Hartmann, L. 1994, AJ, 108, 1872

Kenyon, S. J., \& Hartmann, L. 1987, ApJ, 323, 714

Kessler-Silacci, J., et al. 2006, ApJ, 639, 275

Kessler-Silacci, J. E., et al. 2007, ApJ, 659, 680

Kóspál, Á., Ardila, D. R., Moór, A., \& Ábrahám, P. 2009, ApJ, 700, L73

Lagrange, A.-M., et al. 2010, Science, 329, 57

Lahuis, F., et al. 2006, c2d Spectroscopy Explanatory Supplement, Technical Report (Pasadena, CA: Spitzer Science Center)

Li, A., \& Draine, B. T. 2001, ApJ, 550, L213

Lisse, C. M., Chen, C. H., Wyatt, M. C., \& Morlok, A. 2008, ApJ, 673, 1106

Lisse, C. M., Kraemer, K. E., Nuth, J. A., Li, A., \& Joswiak, D. 2007, Icarus, 187,69
Luhman, K. L., Allen, P. R., Espaillat, C., Hartmann, L., \& Calvet, N. 2010, ApJS, 186, 111

Luhman, K. L., \& Steeghs, D. 2004, ApJ, 609, 917

Malfait, K., Waelkens, C., Waters, L. B. F. M., Vandenbussche, B., Huygen, E., \& de Graauw, M. S. 1998, A\&A, 332, L25

Mamajek, E. E., Lawson, W. A., \& Feigelson, E. D. 1999, ApJ, 516, L77

Meeus, G., Waters, L. B. F. M., Bouwman, J., van den Ancker, M. E., Waelkens, C., \& Malfait, K. 2001, A\&A, 365, 476

Megeath, S. T., Hartmann, L., Luhman, K. L., \& Fazio, G. G. 2005, ApJ, 634, L113

Merín, B., et al. 2007, ApJ, 661, 361

Merín, B., et al. 2010, ApJ, 718, 1200

Min, M., Hovenier, J. W., \& de Koter, A. 2005, A\&A, 432, 909

Min, M., Hovenier, J. W., de Koter, A., Waters, L. B. F. M., \& Dominik, C. 2005, Icarus, 179, 158

Min, M., Hovenier, J. W., Waters, L. B. F. M., \& de Koter, A. 2008, A\&A, 489, 135

Min, M., Waters, L. B. F. M., de Koter, A., Hovenier, J. W., Keller, L. P., \& Markwick-Kemper, F. 2007, A\&A, 462, 667

Oliveira, I., et al. 2009, ApJ, 691, 672

Oliveira, I., et al. 2010, ApJ, 714, 778

Olofsson, J., Augereau, J.-C., van Dishoeck, E. F., Merín, B., Grosso, N., Ménard, F., Blake, G. A., \& Monin, J.-L. 2010, A\&A, 520, A39

Olofsson, J., et al. 2009, A\&A, 507, 327

Pascucci, I., Apai, D., Luhman, K., Henning, T., Bouwman, J., Meyer, M. R., Lahuis, F., \& Natta, A. 2009, ApJ, 696, 143

Pinte, C., et al. 2008, A\&A, 489, 633

Pontoppidan, K. M., \& Brearley, A. J. 2010, in Protoplanetary Dust: Astrophysical and Cosmochemical Perspectives, ed. D. Apai \& D. S. Lauretta (Cambridge: Cambridge Univ. Press), 191

Rieke, G. H., et al. 2005, ApJ, 620, 1010

Sargent, B. A., et al. 2009, ApJS, 182, 477

Schegerer, A. A., Wolf, S., Ratzka, T., \& Leinert, C. 2008, A\&A, 478, 779

Sicilia-Aguilar, A., et al. 2009, ApJ, 701, 1188

Straizys, V., Cernis, K., \& Bartasiute, S. 1996, Balt. Astron., 5, 125

Su, K. Y. L., et al. 2006, ApJ, 653, 675

Udry, S., \& Santos, N. C. 2007, ARA\&A, 45, 397

van Boekel, R., Min, M., Waters, L. B. F. M., de Koter, A., Dominik, C., van den Ancker, M. E., \& Bouwman, J. 2005, A\&A, 437, 189

van Boekel, R., Waters, L. B. F. M., Dominik, C., Bouwman, J., de Koter, A., Dullemond, C. P., \& Paresce, F. 2003, A\&A, 400, L21

van Boekel, R., et al. 2004, Nature, 432, 479

Visser, R., \& Dullemond, C. P. 2010, A\&A, 519, A28

Watson, D. M., et al. 2009, ApJS, 180, 84

Weidenschilling, S. J. 1980, Icarus, 44, 172

Wooden, D., Desch, S., Harker, D., Gail, H.-P., \& Keller, L. 2007, in Protostars and Planets V, ed. B. Reipurth, D. Jewitt, \& K. Keil (Tucson, AZ: Univ. Arizona Press), 815

Wooden, D. H., Harker, D. E., Woodward, C. E., Butner, H. M., Koike, C., Witteborn, F. C., \& McMurtry, C. W. 1999, ApJ, 517, 1034

Zolensky, M. E., et al. 2006, Science, 314, 1735 